Supporting Information

\title{
Enantioselective Dual-Catalysis: A Sequential Michael Addition/Asymmetric Transfer Hydrogenation of $\alpha$-Nitrosulfone and Enones
}

Fengwei Chang, Shitong Wang, Zhitong Zhao, Lijian Wang, Tanyu Cheng, and Guohua Liu* Key Laboratory of Resource Chemistry of Ministry of Education, Shanghai Key Laboratory of Rare Earth Functional Materials, Shanghai Normal University, Shanghai 200234, P. R. China. E-mail: ghliu@shnu.edu.cn

\section{CONTENTS}

Experimental. S2

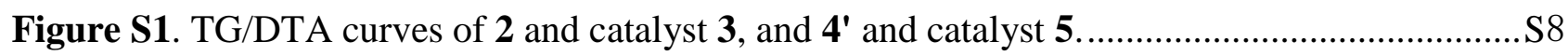

Figure S2. Solid-state ${ }^{13} \mathrm{C}$ CP/MAS NMR spectra of 2 and catalysts 3.....................................S9

Figure S3. Solid-state ${ }^{29}$ Si MAS NMR spectra of catalysts 3 and 5.............................................. S9

Figure S4. (a) TEM images and (b) TEM image with a chemical mapping of 3 showing the

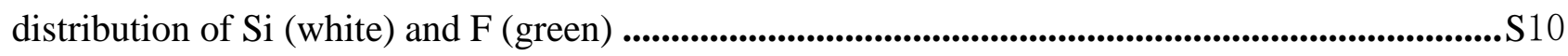

Figure S5. Small-angle powder XRD patterns of $\mathbf{2}$ and catalyst $\mathbf{3}$, and $\mathbf{4}^{\prime}$ and catalyst 5...............S10

Figure S6. Nitrogen adsorption-desorption isotherms of $\mathbf{2}$ and catalyst $\mathbf{3}$, and $\mathbf{4}$ and catalyst 5...S11

Figure S7. Average hydrodynamic diameters distribution measurement of 5..........................S16

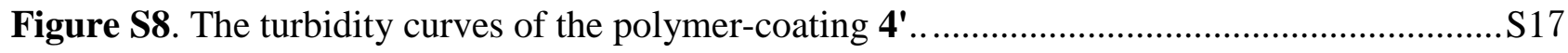

Table S1. Optimizing reaction conditions for the Michael addition reaction. ............................S18

Table S2. Optimizing reaction conditions for the asymmetric transfer hydrogenation. ................S18

Figure S9. HPLC analyses of chiral products .......................................................................................S36

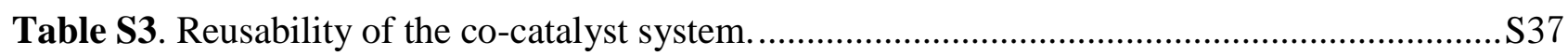

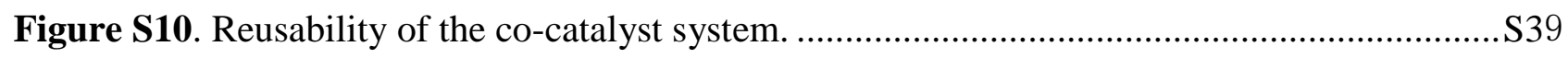

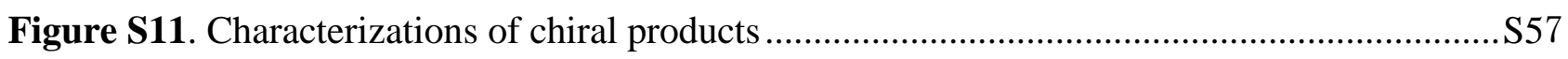




\section{Experimental}

1. General: All experiments, which are sensitive to moisture or air, were carried out under an Ar atmosphere using the standard Schlenk techniques. Tetraethoxysilane (TEOS), 1,4-bis(triethyoxysilyl)ethane, cetyltrimethylammonium bromide (CTAB), fluorocarbon surfactant

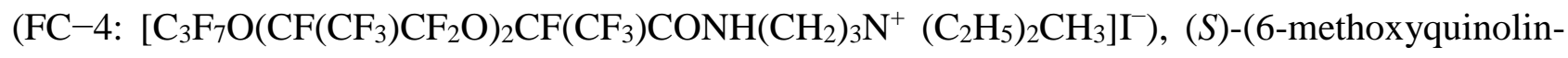
4-yl)((1S,2S,4S,5R)-5-vinylquinuclidin-2-yl)methanamine,

$3-((3,5-$ bis(trifluoromethyl)phenyl)amino)-4-methoxycyclobut-3-ene-1,2-dione, acrylamide, acrylonitrile, 2,2-azobisisobutyronitrile, (Mesitylene $\left.\mathrm{RuCl}_{2}\right)_{2}$ were purchased from Sigma-Aldrich Company Ltd and used as received. Compound of 1-(4-(N-((1S,2S)-2-amino-1,2-diphenylethyl)sulfamoyl)benzyl)1,4-diazabicyclo[2.2.2] octan-1-ium bromide [Molecular Catalysis. 2018, 455, 103-107] was synthesized according to the reported literature.

2. Characterization: Ru loading amounts in the catalysts were analyzed using an inductively coupled plasma optical emission spectrometer (ICP-OES, Varian VISTA-MPX). Scanning electron microscopy (SEM) images were obtained using a JEOL JSM-6380LV microscope operating at 20 $\mathrm{kV}$. Transmission electron microscopy (TEM) images were performed on a JEOL JEM2010 electron microscope at an acceleration voltage of $220 \mathrm{kV}$. Nitrogen adsorption isotherms were measured at 77 $\mathrm{K}$ with a Quantachrome Nova 4000 analyzer. The samples were measured after being outgassed at $423 \mathrm{~K}$ overnight. Pore size distributions were calculated by using the BJH model. The specific surface areas $\left(S_{\mathrm{BET}}\right)$ of samples were determined from the linear parts of BET plots $\left(p / p_{0}=0.05-1.00\right)$. Solidstate NMR experiments were explored on a Bruker AVANCE spectrometer at a magnetic field strength of $9.4 \mathrm{~T}$ with ${ }^{1} \mathrm{H}$ frequency of $400.1 \mathrm{MHz},{ }^{13} \mathrm{C}$ frequency of $100.5 \mathrm{MHz}$ and ${ }^{29} \mathrm{Si}$ frequency of 79.4 MHz with $4 \mathrm{~mm}$ rotor at two spinning frequency of $5.5 \mathrm{kHz}$ and $8.0 \mathrm{kHz}$, TPPM decoupling is applied in the during the acquisition period. ${ }^{1} \mathrm{H}$ cross-polarization in the solid-state NMR experiments was employed using a contact time of $2 \mathrm{~ms}$ and the pulse lengths of $4 \mu \mathrm{s}$. 


\section{Data of chiral products.}

8a: (1S,4S)-4-nitro-1-phenyl-4-(phenylsulfonyl)pentan-1-ol. White solid, 98\% yield, 99\% eе, 98/2 $d r .{ }^{1} \mathrm{H}$ NMR (400 MHz, Methanol- $\left.d_{4}\right) \delta 7.74(\mathrm{ddt}, J=8.0,3.4,1.8 \mathrm{~Hz}, 3 \mathrm{H}), 7.61-7.52(\mathrm{~m}, 2 \mathrm{H}), 7.28$<smiles>CC(CCC(O)c1ccccc1)(c1ccccc1)[N+](=O)[O-]</smiles>
$-7.10(\mathrm{~m}, 5 \mathrm{H}), 4.52$ (dd, $J=7.8,4.9 \mathrm{~Hz}, 1 \mathrm{H}), 2.47-2.10(\mathrm{~m}, 2 \mathrm{H}), 1.91$ $-1.73(\mathrm{~m}, 4 \mathrm{H}), 1.35(\mathrm{tdd}, J=10.9,7.2,5.1 \mathrm{~Hz}, 1 \mathrm{H}) .{ }^{13} \mathrm{C} \mathrm{NMR}(100 \mathrm{MHz}$, Methanol- $\left.d_{4}\right) \delta 135.11,130.74,128.94,127.99,127.11,125.41,72.54$, 32.49, 30.36, 15.83. HRMS (ESI): $\mathrm{m} / \mathrm{z} \quad[\mathrm{M}+\mathrm{Na}]^{+}$calcd for $\left[\mathrm{C}_{17} \mathrm{H}_{19} \mathrm{NO}_{5} \mathrm{SNa}\right]^{+}$372.0876; found: 372.0876. HPLC (Chiralpak OZ-H, elute: Hexanes/i-PrOH = 90/10, detector: $254 \mathrm{~nm}$, flow rate: $1.0 \mathrm{~mL} / \mathrm{min}, 25^{\circ} \mathrm{C}$ ).

8b: (1S,4S)-1-(3-fluorophenyl)-4-nitro-4-(phenylsulfonyl)pentan-1-ol. White solid, 96\% yield,<smiles>CC(CCC(O)c1cccc(F)c1)([N+](=O)[O-])[Sn](=O)[O-]</smiles>
99\% ee, 96/4 dr. ${ }^{1} \mathrm{H}$ NMR (400 MHz, Methanol- $\left.d_{4}\right) \delta 7.81-6.79(\mathrm{~m}$, $9 \mathrm{H}), 4.54(\mathrm{dd}, J=7.7,4.8 \mathrm{~Hz}, 1 \mathrm{H}), 2.39-2.15(\mathrm{~m}, 2 \mathrm{H}), 1.88-1.71(\mathrm{~m}$, $4 \mathrm{H}), 1.42-1.29(\mathrm{~m}, 1 \mathrm{H}) .{ }^{13} \mathrm{C}$ NMR $\left(100 \mathrm{MHz}\right.$, Methanol- $\left.d_{4}\right) \delta 130.75$, $129.78,128.95,121.20,112.00,106.99,71.76,32.44,30.23,15.84$. HRMS (ESI): $\mathrm{m} / \mathrm{z}[\mathrm{M}+\mathrm{Na}]^{+}$calcd for $\left[\mathrm{C}_{17} \mathrm{H}_{18} \mathrm{FNO}_{5} \mathrm{SNa}\right]^{+}$390.0782; found: 390.0782. HPLC (Chiralpak IC, elute: Hexanes/i-PrOH $=85 / 15$, detector: $254 \mathrm{~nm}$, flow rate: $1.0 \mathrm{~mL} / \mathrm{min}, 25{ }^{\circ} \mathrm{C}$ ).

8c: (1S,4S)-1-(2-fluorophenyl)-4-nitro-4-(phenylsulfonyl)pentan-1-ol. White solid, 90\% yield,<smiles>CC(CCC(O)c1ccccc1F)([N+](=O)[O-])S(=O)(=O)O</smiles>
99\% ee, 94/6dr. ${ }^{1} \mathrm{H}$ NMR (400 MHz, Methanol- $\left.d_{4}\right) \delta 7.87-6.89(\mathrm{~m}, 9 \mathrm{H})$, $4.97-4.88(\mathrm{~m}, 1 \mathrm{H}), 2.56-2.06(\mathrm{~m}, 2 \mathrm{H}), 2.00-1.68(\mathrm{~m}, 4 \mathrm{H}), 1.55-1.36$ $(\mathrm{m}, 1 \mathrm{H}) .{ }^{13} \mathrm{C}$ NMR $\left(100 \mathrm{MHz}\right.$, Methanol- $\left.d_{4}\right) \delta 160.71,158.28,135.14$, 132.84, 130.73, 128.96, 128.76, 126.96, 124.00, 114.77, 106.94, 66.06, 31.37, 30.21, 15.78.HRMS (ESI): $\mathrm{m} / \mathrm{z}[\mathrm{M}+\mathrm{Na}]^{+}$calcd for $\left[\mathrm{C}_{17} \mathrm{H}_{18} \mathrm{FNO}_{5} \mathrm{SNa}\right]^{+}$390.0782; found: 390.0782. HPLC (Chiralpak OZ$\mathrm{H}$, elute: Hexanes $/ i$-PrOH $=90 / 10$, detector: $254 \mathrm{~nm}$, flow rate: $0.8 \mathrm{~mL} / \mathrm{min}, 2{ }^{\circ} \mathrm{C}$ ).

8d: (1S,4S)-1-(4-chlorophenyl)-4-nitro-4-(phenylsulfonyl)pentan-1-ol. White solid, 95\% yield,<smiles>CC(CC[C@H](O)c1ccc(Cl)cc1)([N+](=O)[O-])[Sn](=O)[O-]</smiles>
99\% ee, 94/6 $d r .{ }^{1} \mathrm{H}$ NMR (400 MHz, Methanol- $\left.d_{4}\right) \delta 7.81-7.68(\mathrm{~m}$, $3 \mathrm{H}), 7.58(\mathrm{dd}, J=8.5,7.3 \mathrm{~Hz}, 2 \mathrm{H}), 7.21(\mathrm{q}, J=8.6 \mathrm{~Hz}, 4 \mathrm{H}), 4.60-$ $4.47(\mathrm{~m}, 1 \mathrm{H}), 2.24(\mathrm{dd}, J=9.1,7.5 \mathrm{~Hz}, 2 \mathrm{H}), 1.82(\mathrm{~s}, 3 \mathrm{H}), 1.80-1.72$ $(\mathrm{m}, 1 \mathrm{H}), 1.40-1.25(\mathrm{~m}, 1 \mathrm{H}) .{ }^{13} \mathrm{C}$ NMR $\left(100 \mathrm{MHz}\right.$, Methanol- $\left.d_{4}\right) \delta$ 142.89, 135.11, 132.69, 130.74, 129.57, 128.94, 128.04, 127.09, 106.96, 71.76, 32.39, 30.27, 15.82 . HRMS (ESI): $\mathrm{m} / \mathrm{z}[\mathrm{M}+\mathrm{Na}]^{+}$calcd for $\left[\mathrm{C}_{17} \mathrm{H}_{18} \mathrm{ClNO}_{5} \mathrm{SNa}\right]^{+}$406.0486; found: 406.0486. HPLC (Chiralpak OZ-H, elute: Hexanes $/ i-\mathrm{PrOH}=90 / 10$, detector: $254 \mathrm{~nm}$, flow rate: $1.0 \mathrm{~mL} / \mathrm{min}, 25^{\circ} \mathrm{C}$, ). 
8e: (1S,4S)-1-(2-chlorophenyl)-4-nitro-4-(phenylsulfonyl)pentan-1-ol. White solid, 95\% yield,<smiles>CC(CCC(O)c1ccccc1Cl)([N+](=O)[O-])S(=O)(=O)O</smiles>
96\% ee, 93/7 $d r .{ }^{1} \mathrm{H}$ NMR (400 MHz, Methanol- $\left.d_{4}\right) \delta 7.95-7.09(\mathrm{~m}, 9 \mathrm{H})$, $5.07-4.92(\mathrm{~m}, 1 \mathrm{H}), 2.54-2.12(\mathrm{~m}, 2 \mathrm{H}), 2.04-1.71(\mathrm{~m}, 4 \mathrm{H}), 1.56-1.37$ $(\mathrm{m}, 1 \mathrm{H}) .{ }^{13} \mathrm{C}$ NMR $\left(100 \mathrm{MHz}\right.$, Methanol- $\left.d_{4}\right) \delta 141.56,135.15,132.85$, $131.14,130.74,128.98,128.91,128.31,126.86,126.82,106.99,68.70$, 31.13, 30.30, 15.74.HRMS (ESI): $\mathrm{m} / \mathrm{z}[\mathrm{M}+\mathrm{Na}]^{+}$calcd for $\left[\mathrm{C}_{17} \mathrm{H}_{18} \mathrm{ClNO}_{5} \mathrm{SNa}\right]^{+}$406.0486; found: 406.0486. HPLC (Chiralpak OZ-H, elute: Hexanes/i-PrOH =90/10, detector: $254 \mathrm{~nm}$, flow rate: 0.5 $\left.\mathrm{mL} / \mathrm{min}, 25^{\circ} \mathrm{C}\right)$.

8f: (1S,4S)-1-(4-bromophenyl)-4-nitro-4-(phenylsulfonyl)pentan-1-ol. White solid, 93\% yield,<smiles>CC(CC[C@H](O)c1ccc(Br)cc1)([N+](=O)[O-])[Sn](=O)[O-]</smiles>
99\% ee, 96/4 $d r .{ }^{1} \mathrm{H}$ NMR (400 MHz, Methanol- $\left.d_{4}\right) \delta 7.80-7.69(\mathrm{~m}$, $3 \mathrm{H}), 7.62-7.54(\mathrm{~m}, 2 \mathrm{H}), 7.43-7.35(\mathrm{~m}, 2 \mathrm{H}), 7.13(\mathrm{~d}, J=8.4 \mathrm{~Hz}$, $2 \mathrm{H}), 4.50(\mathrm{dd}, J=7.7,5.1 \mathrm{~Hz}, 1 \mathrm{H}), 2.31-2.17(\mathrm{~m}, 2 \mathrm{H}), 1.82(\mathrm{~s}, 3 \mathrm{H})$, $1.81-1.73(\mathrm{~m}, 1 \mathrm{H}), 1.38-1.26(\mathrm{~m}, 1 \mathrm{H}) .{ }^{13} \mathrm{C}$ NMR $(100 \mathrm{MHz}$, Methanol- $\left.d_{4}\right) \delta 143.37,135.12,132.82,131.06,130.74,128.95,127.43,120.66,106.96,71.79,32.33$, 30.26, 15.81. HRMS (ESI): $\mathrm{m} / \mathrm{z}[\mathrm{M}+\mathrm{Na}]^{+}$calcd for $\left[\mathrm{C}_{17} \mathrm{H}_{18} \mathrm{BrNO}_{5} \mathrm{SNa}\right]^{+} 449.9981$; found: 449.9981. HPLC (Chiralpak OZ-H, elute: Hexanes/ $i$-PrOH $=90 / 10$, detector: $254 \mathrm{~nm}$, flow rate: $1.0 \mathrm{~mL} / \mathrm{min}$, $\left.25^{\circ} \mathrm{C}\right)$.

8g: (1S,4S)-1-(3-bromophenyl)-4-nitro-4-(phenylsulfonyl)pentan-1-ol. White solid, 96\% yield,<smiles>CC(CCC(O)c1cccc(Br)c1)([N+](=O)[O-])[Sn](=O)[O-]</smiles>
99\% ee, 96/4dr. ${ }^{1} \mathrm{H}$ NMR (400 MHz, Methanol- $\left.d_{4}\right) \delta 7.85-7.07(\mathrm{~m}$, $9 \mathrm{H}), 4.51(\mathrm{dd}, J=7.8,4.9 \mathrm{~Hz}, 1 \mathrm{H}), 2.25(\mathrm{ddt}, J=14.2,10.0,5.1 \mathrm{~Hz}$, $2 \mathrm{H}), 1.94-1.68(\mathrm{~m}, 4 \mathrm{H}), 1.42-1.28(\mathrm{~m}, 1 \mathrm{H}) .{ }^{13} \mathrm{C} \mathrm{NMR}(100 \mathrm{MHz}$, Methanol- $\left.d_{4}\right) \delta 146.74,135.14,132.88,130.74,130.02,129.81,128.95,128.48,124.25,121.99$, 106.88, 71.48, 32.26, 29.87, 15.79. HRMS (ESI): $\mathrm{m} / \mathrm{z}[\mathrm{M}+\mathrm{Na}]^{+}$calcd for $\left[\mathrm{C}_{17} \mathrm{H}_{18} \mathrm{BrNO}_{5} \mathrm{SNa}^{+}\right.$ 449.9981; found: 449.9981. HPLC (Chiralpak OD-3, elute: Hexanes/i-PrOH =85/15, detector: 254 $\mathrm{nm}$, flow rate: $1.0 \mathrm{~mL} / \mathrm{min}, 25^{\circ} \mathrm{C}$ ).

8h: (1S,4S)-1-(4-iodophenyl)-4-nitro-4-(phenylsulfonyl)pentan-1-ol. White solid, 92\% yield, 99\%<smiles></smiles>
$e e, 91 / 9 d r .{ }^{1} \mathrm{H}$ NMR (400 MHz, Methanol- $\left.d_{4}\right) \delta 7.79-7.68(\mathrm{~m}, 3 \mathrm{H})$, $7.62-7.54(\mathrm{~m}, 4 \mathrm{H}), 6.99(\mathrm{~d}, J=8.3 \mathrm{~Hz}, 2 \mathrm{H}), 4.52(\mathrm{ddd}, J=21.7,7.3$, $5.1 \mathrm{~Hz}, 1 \mathrm{H}), 2.30-2.13(\mathrm{~m}, 2 \mathrm{H}), 1.82(\mathrm{~s}, 3 \mathrm{H}), 1.81-1.72(\mathrm{~m}, 1 \mathrm{H}), 1.38$ $-1.25(\mathrm{~m}, 1 \mathrm{H}) .{ }^{13} \mathrm{C}$ NMR $\left(100 \mathrm{MHz}\right.$, Methanol- $\left.d_{4}\right) \delta 143.93,137.16$, 135.12, 132.81, 130.74, 128.95, 127.60, 106.95, 91.91, 71.88, 32.29, 30.27, 15.79. HRMS (ESI): m/z $[\mathrm{M}+\mathrm{Na}]^{+}$calcd for $\left[\mathrm{C}_{17} \mathrm{H}_{18} \mathrm{INO}_{5} \mathrm{SNa}\right]^{+}$497.9843; found: 497.9843. HPLC (Chiralpak OZ-H, elute: Hexanes $/ i$-PrOH $=90 / 10$, detector: $254 \mathrm{~nm}$, flow rate: $1.0 \mathrm{~mL} / \mathrm{min}, 25^{\circ} \mathrm{C}$ ). 
8i: (1S,4S)-4-nitro-1-(4-nitrophenyl)-4-(phenylsulfonyl)pentan-1-ol. White solid, 92\% yield, 99\%<smiles>CC(CC[C@H](O)c1ccc([N+](=O)[O-])cc1)(Oc1ccccc1)[N+](=O)[O-]</smiles>
$e e, 92 / 8 d r .{ }^{1} \mathrm{H}$ NMR (400 MHz, Methanol- $\left.d_{4}\right) \delta 8.11(\mathrm{~d}, J=8.8 \mathrm{~Hz}$, 2H), $7.83-7.69(\mathrm{~m}, 3 \mathrm{H}), 7.58(\mathrm{t}, J=7.9 \mathrm{~Hz}, 2 \mathrm{H}), 7.47(\mathrm{~d}, J=8.4$ $\mathrm{Hz}, 2 \mathrm{H}), 4.66(\mathrm{dd}, J=7.8,4.7 \mathrm{~Hz}, 1 \mathrm{H}), 2.30(\mathrm{p}, J=8.7 \mathrm{~Hz}, 2 \mathrm{H}), 1.95$ $-1.72(\mathrm{~m}, 4 \mathrm{H}), 1.39(\mathrm{dtd}, J=14.1,10.4,9.2,4.9 \mathrm{~Hz}, 1 \mathrm{H}) \cdot{ }^{13} \mathrm{C} \mathrm{NMR}$ $\left(100 \mathrm{MHz}\right.$, Methanol- $\left.d_{4}\right) \delta 151.89,135.13,132.83,130.76,130.73,126.49,126.42,123.09,106.90$, 71.46, 32.39, 30.13, 15.85. HRMS (ESI): $\mathrm{m} / \mathrm{z}[\mathrm{M}+\mathrm{Na}]^{+}$calcd for $\left[\mathrm{C}_{17} \mathrm{H}_{18} \mathrm{~N}_{2} \mathrm{O}_{7} \mathrm{SNa}\right]^{+} 417.0727$; found: 417.0727. HPLC (Chiralpak OZ-H, elute: Hexanes/i-PrOH =85/15, detector: $254 \mathrm{~nm}$, flow rate: $1.0 \mathrm{~mL} / \mathrm{min}, 25^{\circ} \mathrm{C}$ ).

8j: 4-((1S,4S)-1-hydroxy-4-nitro-4-(phenylsulfonyl)pentyl)benzonitrile. White solid, 93\% yield,<smiles></smiles>
$99 \%$ ee, $92 / 8 d r .{ }^{1} \mathrm{H}$ NMR (400 MHz, Methanol- $\left.d_{4}\right) \delta 7.80-7.72(\mathrm{~m}$, $3 \mathrm{H}), 7.64-7.54(\mathrm{~m}, 4 \mathrm{H}), 7.41(\mathrm{~d}, J=8.1 \mathrm{~Hz}, 2 \mathrm{H}), 4.61(\mathrm{dd}, J=8.1$, $4.8 \mathrm{~Hz}, 1 \mathrm{H}), 2.39-2.20(\mathrm{~m}, 2 \mathrm{H}), 1.82(\mathrm{~s}, 3 \mathrm{H}), 1.80-1.72(\mathrm{~m}, 1 \mathrm{H})$, $1.44-1.30(\mathrm{~m}, 1 \mathrm{H}) .{ }^{13} \mathrm{C}$ NMR $\left(100 \mathrm{MHz}\right.$, Methanol- $\left.d_{4}\right) \delta 150.02$, $135.15,132.83$, 131.92, 130.75, 128.96, 126.44, 118.29, 110.70, 106.91, 71.65, 32.35, 30.13, 15.85 . HRMS (ESI): $\mathrm{m} / \mathrm{z}[\mathrm{M}+\mathrm{Na}]^{+}$calcd for $\left[\mathrm{C}_{18} \mathrm{H}_{18} \mathrm{~N}_{2} \mathrm{O}_{5} \mathrm{SNa}\right]^{+}$397.0829; found: 397.0829. HPLC (Chiralpak OZ-H, elute: Hexanes/i-PrOH $=90 / 10$, detector: $254 \mathrm{~nm}$, flow rate: $1.0 \mathrm{~mL} / \mathrm{min}, 25^{\circ} \mathrm{C}$ ).

8k: (1S,4S)-1-(3-methoxyphenyl)-4-nitro-4-(phenylsulfonyl)pentan-1-ol. White solid, 94\% yield,<smiles>COc1cccc(C(O)CC[C@](C)(O)S(=O)(=O)Oc2ccccc2)c1</smiles>
99\% ee, 95/5 $d r .{ }^{1} \mathrm{H}$ NMR (400 MHz, Methanol- $\left.d_{4}\right) \delta 7.74(\mathrm{dd}, J=$ 7.7, $5.6 \mathrm{~Hz}, 3 \mathrm{H}), 7.57(\mathrm{t}, J=7.9 \mathrm{~Hz}, 2 \mathrm{H}), 7.13(\mathrm{t}, J=7.9 \mathrm{~Hz}, 1 \mathrm{H})$, $6.81-6.68(\mathrm{~m}, 3 \mathrm{H}), 4.60-4.43(\mathrm{~m}, 1 \mathrm{H}), 3.70(\mathrm{~s}, 3 \mathrm{H}), 2.34-2.14$ $(\mathrm{m}, 2 \mathrm{H}), 1.82(\mathrm{~s}, 4 \mathrm{H}), 1.41-1.28(\mathrm{~m}, 1 \mathrm{H}) .{ }^{13} \mathrm{C}$ NMR (100 MHz, Methanol-d $) \delta 159.86,145.56$, 135.09, 132.89, 130.73, 129.02, 128.92, 117.73, 112.59, 110.90, 72.42, 54.23, 32.40, 30.32, 15.80. HRMS (ESI): $\mathrm{m} / \mathrm{z}[\mathrm{M}+\mathrm{Na}]^{+}$calcd for $\left[\mathrm{C}_{18} \mathrm{H}_{21} \mathrm{NO}_{6} \mathrm{SNa}\right]^{+}$402.0982; found: 402.0982. HPLC (Chiralpak OZ-H, elute: Hexanes/i-PrOH $=85 / 15$, detector: $254 \mathrm{~nm}$, flow rate: $1.0 \mathrm{~mL} / \mathrm{min}, 25^{\circ} \mathrm{C}$ ).

81: (1S,4S)-1-(4-methoxyphenyl)-4-nitro-4-(phenylsulfonyl)pentan-1-ol. White solid, 91\% yield,<smiles>COc1ccc([C@H](O)CCC(C)(C)S(=O)(=O)Oc2ccccc2)cc1</smiles>
99\% ee, 92/8 $d r .{ }^{1} \mathrm{H}$ NMR (400 MHz, Methanol- $\left.d_{4}\right) \delta 7.80-7.68(\mathrm{~m}$, $3 \mathrm{H}), 7.57(\mathrm{dd}, J=8.5,7.4 \mathrm{~Hz}, 2 \mathrm{H}), 7.17-7.06(\mathrm{~m}, 2 \mathrm{H}), 6.78(\mathrm{~d}, J=$ $8.7 \mathrm{~Hz}, 2 \mathrm{H}), 4.46(\mathrm{dd}, J=7.4,5.4 \mathrm{~Hz}, 1 \mathrm{H}), 3.70(\mathrm{~s}, 3 \mathrm{H}), 2.30-2.10$ $(\mathrm{m}, 2 \mathrm{H}), 1.82(\mathrm{~d}, J=4.7 \mathrm{~Hz}, 4 \mathrm{H}), 1.38-1.27(\mathrm{~m}, 1 \mathrm{H}) .{ }^{13} \mathrm{C}$ NMR $(100$ MHz, Methanol- $\left.d_{4}\right) \delta 159.17,135.75,135.07,132.93,130.72,128.93,126.70,113.40,106.94,72.18$, 54.31, 32.29, 30.14, 15.83. HRMS (ESI): $\mathrm{m} / \mathrm{z}[\mathrm{M}+\mathrm{Na}]^{+}$calcd for $\left[\mathrm{C}_{18} \mathrm{H}_{21} \mathrm{NO}_{6} \mathrm{SNa}\right]^{+}$402.0982; found: 402.0982. HPLC (Chiralpak IC, elute: Hexanes/i-PrOH $=85 / 15$, detector: $254 \mathrm{~nm}$, flow rate: 1.0 $\left.\mathrm{mL} / \mathrm{min}, 25^{\circ} \mathrm{C}\right)$. 
8m: (1S,4S)-4-nitro-4-(phenylsulfonyl)-1-(p-tolyl)pentan-1-ol. White solid, 94\% yield, 99\% ee,<smiles></smiles>
94/6dr. ${ }^{1} \mathrm{H}$ NMR (400 MHz, Methanol- $\left.d_{4}\right) \delta 7.95-6.95(\mathrm{~m}, 9 \mathrm{H}), 4.59-$ $4.39(\mathrm{~m}, 1 \mathrm{H}), 2.60-1.11(\mathrm{~m}, 10 \mathrm{H}) .{ }^{13} \mathrm{C}$ NMR $\left(100 \mathrm{MHz}\right.$, Methanol- $\left.d_{4}\right)$ $\delta 140.84,136.85,135.07,132.88,130.74,128.93,128.60,125.40$, 107.05, 72.44, 32.40, 30.39, 19.74, 15.84. HRMS (ESI): m/z [M+Na] ${ }^{+}$ calcd for $\left[\mathrm{C}_{18} \mathrm{H}_{21} \mathrm{NO}_{5} \mathrm{SNa}\right]^{+}$386.1033; found: 386.1033. HPLC (Chiralpak OD-3, elute: Hexanes/i$\mathrm{PrOH}=85 / 15$, detector: $254 \mathrm{~nm}$, flow rate: $1.0 \mathrm{~mL} / \mathrm{min}, 25^{\circ} \mathrm{C}$ ).

8n: (1S,4S)-4-nitro-4-(phenylsulfonyl)-1-(m-tolyl)pentan-1-ol. White solid, $96 \%$ yield, $99 \% e e$,<smiles>Cc1cccc([C@H](O)CC[C@@](C)([O+]c2ccccc2)[N+](=O)[O-])c1</smiles>
96/4 $d r .{ }^{1} \mathrm{H}$ NMR (400 MHz, Methanol- $\left.d_{4}\right) \delta 7.89-6.93(\mathrm{~m}, 9 \mathrm{H}), 4.47$ $(\mathrm{dd}, J=7.6,5.1 \mathrm{~Hz}, 1 \mathrm{H}), 2.42-2.08(\mathrm{~m}, 5 \mathrm{H}), 1.93-1.73(\mathrm{~m}, 4 \mathrm{H}), 1.42$ $-1.25(\mathrm{~m}, 1 \mathrm{H}) .{ }^{13} \mathrm{C}$ NMR (100 MHz, Methanol- $\left.d_{4}\right) \delta 143.84,137.70$, 135.09, 132.91, 130.74, 128.92, 127.91, 127.77, 126.03, 122.52, 107.06, 72.59, 32.46, 30.40, 20.10, 15.81. HRMS (ESI): $\mathrm{m} / \mathrm{z}[\mathrm{M}+\mathrm{Na}]^{+}$calcd for $\left[\mathrm{C}_{18} \mathrm{H}_{21} \mathrm{NO}_{5} \mathrm{SNa}\right]^{+}$386.1033; found: 386.1033. HPLC (Chiralpak OZ-H, elute: Hexanes/i-PrOH =75/25, detector: $254 \mathrm{~nm}$, flow rate: $1.0 \mathrm{~mL} / \mathrm{min}, 25^{\circ} \mathrm{C}$ ).

8o: $(1 S, 4 S)-1-($ naphthalen-2-yl)-4-nitro-4-(phenylsulfonyl)pentan-1-ol. White solid, 95\% yield,<smiles>CC(CCC(O)c1ccc2ccccc2c1)([N+](=O)[O-])S(=O)(=O)c1ccccc1</smiles>
99\% ee, 95/5 $d r .{ }^{1} \mathrm{H}$ NMR (400 MHz, Methanol- $\left.d_{4}\right) \delta 7.79-7.58(\mathrm{~m}$, $7 \mathrm{H}), 7.47-7.30(\mathrm{~m}, 5 \mathrm{H}), 4.70(\mathrm{dd}, J=7.1,5.6 \mathrm{~Hz}, 1 \mathrm{H}), 2.34-2.15$ $(\mathrm{m}, 2 \mathrm{H}), 1.96-1.86(\mathrm{~m}, 1 \mathrm{H}), 1.84(\mathrm{~s}, 3 \mathrm{H}), 1.45(\mathrm{ddt}, J=12.5,9.9$, $5.4 \mathrm{~Hz}, 1 \mathrm{H}) .{ }^{13} \mathrm{C}$ NMR $\left(100 \mathrm{MHz}\right.$, Methanol- $\left.d_{4}\right) \delta 141.23,134.98$, 133.38, 133.31, 133.07, 132.76, 130.65, 128.79, 127.83, 127.58, 127.25, 125.74, 125.44, 124.24, 123.49, 72.63, 32.18, 30.48, 15.73. HRMS (ESI): $\mathrm{m} / \mathrm{z}[\mathrm{M}+\mathrm{Na}]^{+}$calcd for $\left[\mathrm{C}_{21} \mathrm{H}_{21} \mathrm{NO}_{5} \mathrm{SNa}^{+}\right.$ 422.1033; found: 422.1033. HPLC (Chiralpak IC, elute: Hexanes/ $i$-PrOH =90/10, detector: $254 \mathrm{~nm}$, flow rate: $1.0 \mathrm{~mL} / \mathrm{min}, 25^{\circ} \mathrm{C}$ ).

8p: (1S,4S)-1-(furan-2-yl)-4-nitro-4-(phenylsulfonyl)pentan-1-ol. White solid, 95\% yield, 96\% ee,<smiles>CC(CCC(O)c1ccco1)([N+](=O)[O-])[Sn](=O)[O-]</smiles>
95/5dr. ${ }^{1} \mathrm{H}$ NMR (400 MHz, Methanol- $\left.d_{4}\right) \delta 7.98-7.33(\mathrm{~m}, 6 \mathrm{H}), 6.46-$ $6.13(\mathrm{~m}, 2 \mathrm{H}), 4.59(\mathrm{dd}, J=7.5,5.1 \mathrm{~Hz}, 1 \mathrm{H}), 2.47-2.24(\mathrm{~m}, 2 \mathrm{H}), 2.03-$ $1.94(\mathrm{~m}, 1 \mathrm{H}), 1.92(\mathrm{~s}, 3 \mathrm{H}), 1.66-1.49(\mathrm{~m}, 1 \mathrm{H}) .{ }^{13} \mathrm{C}$ NMR $(100 \mathrm{MHz}$, Methanol- $\left.d_{4}\right) \delta 155.95,141.76,135.15,132.92,130.75,128.97,124.07,109.67,105.76,66.07,29.99$, 29.78, 15.93. HRMS (ESI): $\mathrm{m} / \mathrm{z}[\mathrm{M}+\mathrm{Na}]^{+}$calcd for $\left[\mathrm{C}_{15} \mathrm{H}_{17} \mathrm{NO}_{6} \mathrm{SNa}\right]^{+} 362.0669$; found: 362.0669 . HPLC (Chiralpak IC, elute: Hexanes $/ i$-PrOH $=95 / 5$, detector: $254 \mathrm{~nm}$, flow rate: $1.0 \mathrm{~mL} / \mathrm{min}, 25^{\circ} \mathrm{C}$ ). 
8q: (1S,4S)-4-nitro-4-(phenylsulfonyl)-1-(thiophen-2-yl)pentan-1-ol. White solid, 96\% yield,<smiles>C[C@](CCC(O)c1cccs1)(O[S+](=O)c1ccccc1)[N+](=O)[O-]</smiles>
99\% ee, 95/5dr. ${ }^{1} \mathrm{H}$ NMR (400 MHz, Methanol- $\left.d_{4}\right) \delta 7.87-7.70(\mathrm{~m}, 3 \mathrm{H})$, (s, 3H), $1.58-1.37(\mathrm{~m}, 1 \mathrm{H}) .{ }^{13} \mathrm{C}$ NMR (100 MHz, Methanol- $\left.d_{4}\right) \delta 147.95,135.16,132.85,130.76$, 128.98, 126.17, 123.99, 123.26, 106.96, 68.50, 32.86, 30.20, 15.98. HRMS (ESI): m/z [M+Na] ${ }^{+}$calcd for $\left.\mathrm{C}_{15} \mathrm{H}_{17} \mathrm{NO}_{5} \mathrm{~S}_{2} \mathrm{Na}\right]^{+}$378.0440; found: 378.0440. HPLC (Chiralpak OD-3, elute: Hexanes/i-PrOH $=85 / 15$, detector: $254 \mathrm{~nm}$, flow rate: $1.0 \mathrm{~mL} / \mathrm{min}, 25^{\circ} \mathrm{C}$ ).

8r: (1S,4S)-1-(3, 9-dihydropyren-4-yl)-4-nitro-4-(phenylsulfonyl)pentan-1-ol. White solid, 8\%<smiles>CC([O-])(CCC(O)c1ccc2ccc3cccc4ccc1c2c34)[O+]=O</smiles>
yield, 96\% ee, 55/45dr. ${ }^{1} \mathrm{H}$ NMR (400 MHz, Methanol- $\left.d_{4}\right) \delta 8.33-7.91$ (m, 9H), $7.68-7.11(\mathrm{~m}, 5 \mathrm{H}), 5.73(\mathrm{dd}, J=7.2,5.2 \mathrm{~Hz}, 1 \mathrm{H}), 2.60-2.06$ $(\mathrm{m}, 3 \mathrm{H}), 1.85(\mathrm{~d}, J=1.8 \mathrm{~Hz}, 3 \mathrm{H}), 1.82-1.59(\mathrm{~m}, 1 \mathrm{H}) .{ }^{13} \mathrm{C}$ NMR $(100$ MHz, Methanol- $\left.d_{4}\right) \delta 137.37,134.87,134.65,131.39,130.54,130.44,128.74,128.53,127.35,127.13$, 126.80, 125.71, 124.94, 124.70, 124.64, 123.24, 123.02, 122.01, 121.86, 107.04, 68.83, 32.63, 32.47, 30.75, 30.58, 15.55. HRMS (ESI): $\mathrm{m} / \mathrm{z}[\mathrm{M}+\mathrm{Na}]^{+}$calcd for $\left[\mathrm{C}_{27} \mathrm{H}_{25} \mathrm{NO}_{5} \mathrm{SNa}\right]^{+} 498.1346$; found: 498.1346. HPLC (Chiralpak IC, elute: Hexanes $/ i-\mathrm{PrOH}=90 / 10$, detector: $254 \mathrm{~nm}$, flow rate: $\left.0.7 \mathrm{~mL} / \mathrm{min}, 25^{\circ} \mathrm{C}\right)$. 


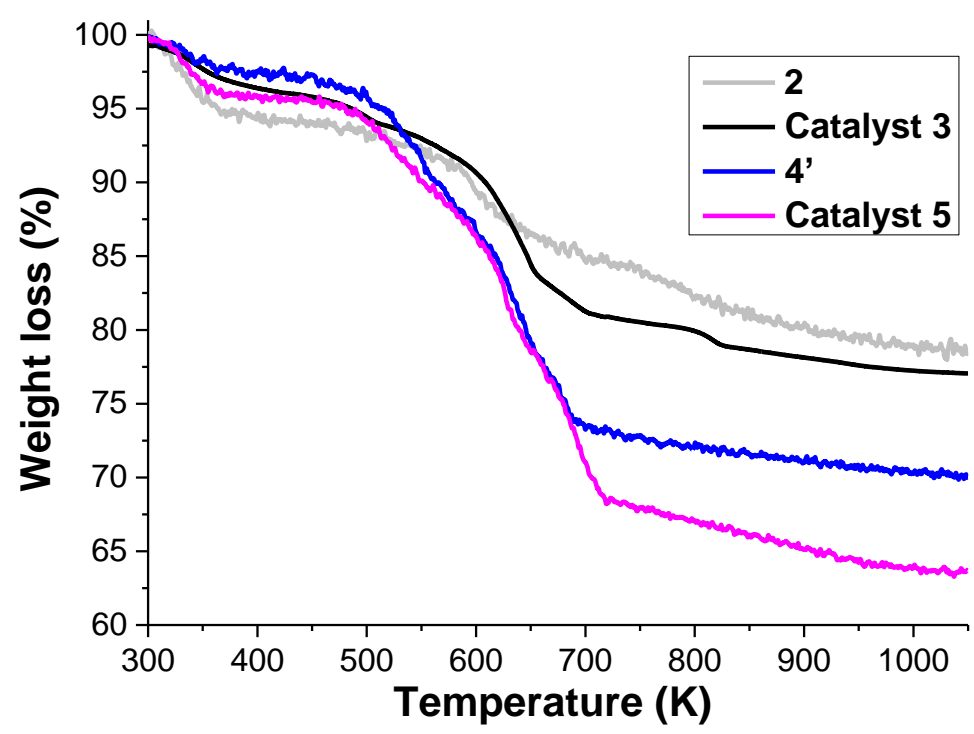

Figure S1. TG/DTA curves of $\mathbf{2}$ and catalyst 3, and 4' and catalyst 5 .

Explanation: The TG/DTA curves of 2-3, 4', and $\mathbf{5}$ were treated in the air as shown above. Besides weight loss before $440 \mathrm{~K}$ with for the release of physical adsorption water, the comprehensive weight loss between $440 \mathrm{~K}$ and $1100 \mathrm{~K}$ could be assigned to the oxidation of organic molecules (including the part of the residual surfactant, the entrapped organic moiety and/or alkyl-linked polymers). The total weight loss for the oxidation of organic molecules of the extracted samples $\mathbf{2 - 3}, \mathbf{4}$ ', and $\mathbf{5}$ are $15.28 \%, 18.69 \%, 26.56 \%$, and $31.68 \%$, respectively.

As compared to the weight loss of $\mathbf{2}$ with catalyst $\mathbf{3}$, the weight loss of the entrapped chiral organic squaramide moieties was $3.41 \%$ (18.69-15.28) per $100 \%$ materials. This finding means that the mole amounts of squaramides in catalyst 3 is $0.005411 \mathrm{~mol} \%(\mathrm{Mr}=630.21)$, demonstrating the $34.10 \mathrm{mg}$ ( $0.05411 \mathrm{mmol}$ of squaramide) of the squaramide-loading per gram of $\mathbf{3}$.

As compared to the weight loss of $\mathbf{2}$ with $\mathbf{4}^{\prime}$, the weight loss of the polymer-coating layer in $\mathbf{5}$ was $11.28 \%$ (26.56-15.28) per $100 \%$ materials. This finding means that the mass amounts of the polymercoating layer in $\mathbf{5}$ is $11.28 \%$.

As compared to the weight loss of $4^{\prime}$ with catalyst $\mathbf{5}$, the weight loss of the entrapped chiral organic part of MesArDPEN plus $\mathrm{Cl}$ moieties was 5.12\% (31.68-26.56) per 100\% materials. This finding means that the mole amounts of the entrapped chiral organic part in catalyst $\mathbf{5}$ are $0.007219 \mathrm{~mol} \%$ $(\mathrm{Mr}=709.20)$, demonstrating the $58.56 \mathrm{mg}(0.07219 \mathrm{mmol}$ of the entrapped MesRuArDPEN $(\mathrm{Mr}=$ 811.10)) of the chiral MesRuArDPEN loading per gram of 5. This finding is accorded with that (7.39 $\mathrm{mg}(0.07256 \mathrm{mmol}$ of $\mathrm{Ru}$ in $\mathbf{5}))$ detected by ICP analysis. 


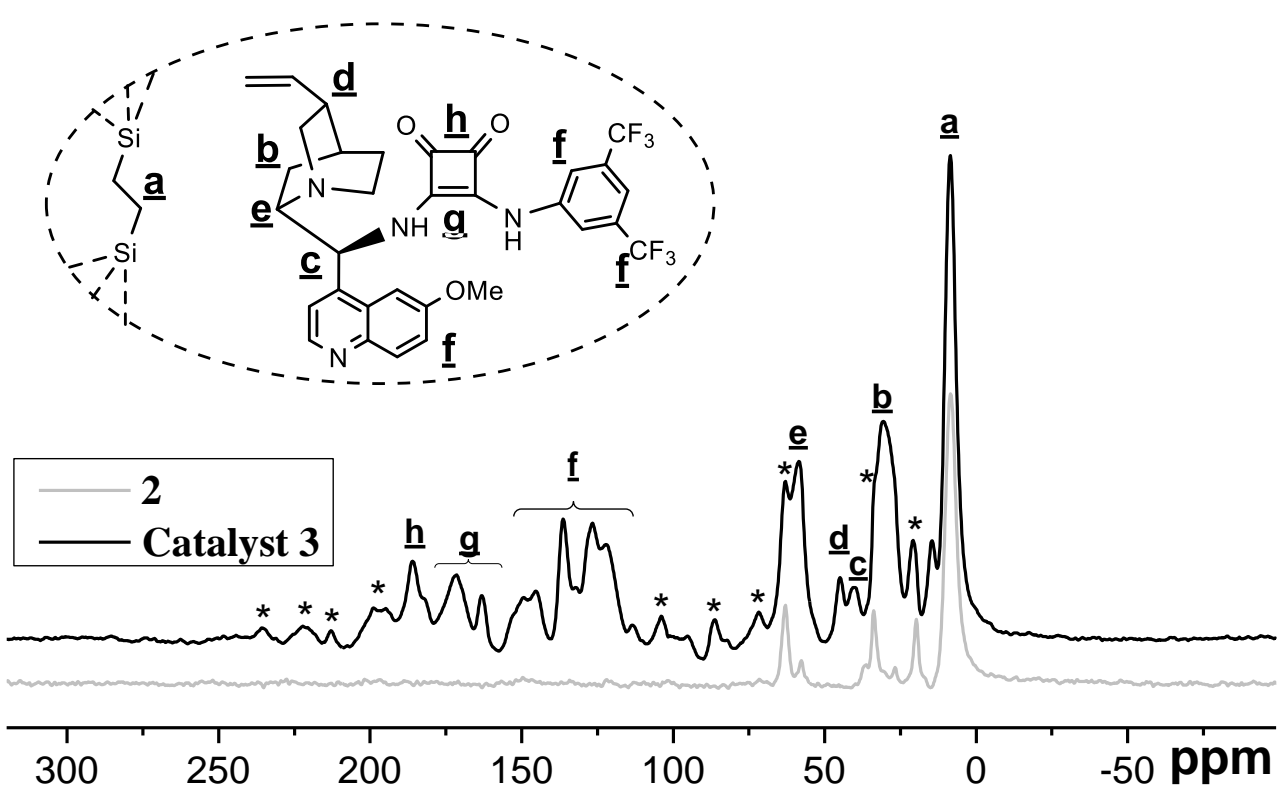

Figure S2. Solid-state ${ }^{13} \mathrm{C}$ CP/MAS NMR spectra of 2 and catalysts 3 .

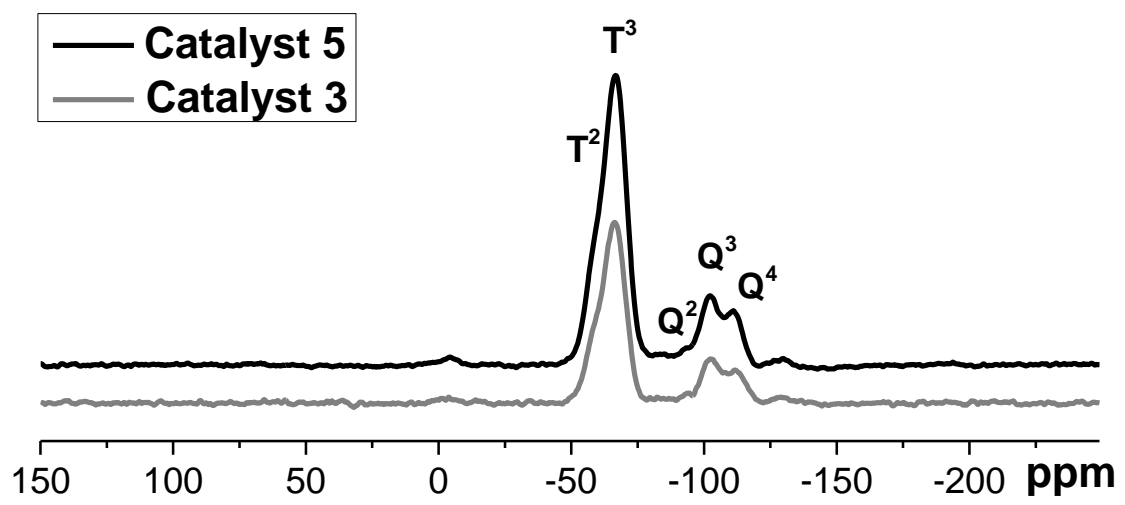

Figure S3. Solid--state ${ }^{29}$ Si MAS NMR spectra of catalysts 3 and $\mathbf{5}$. 

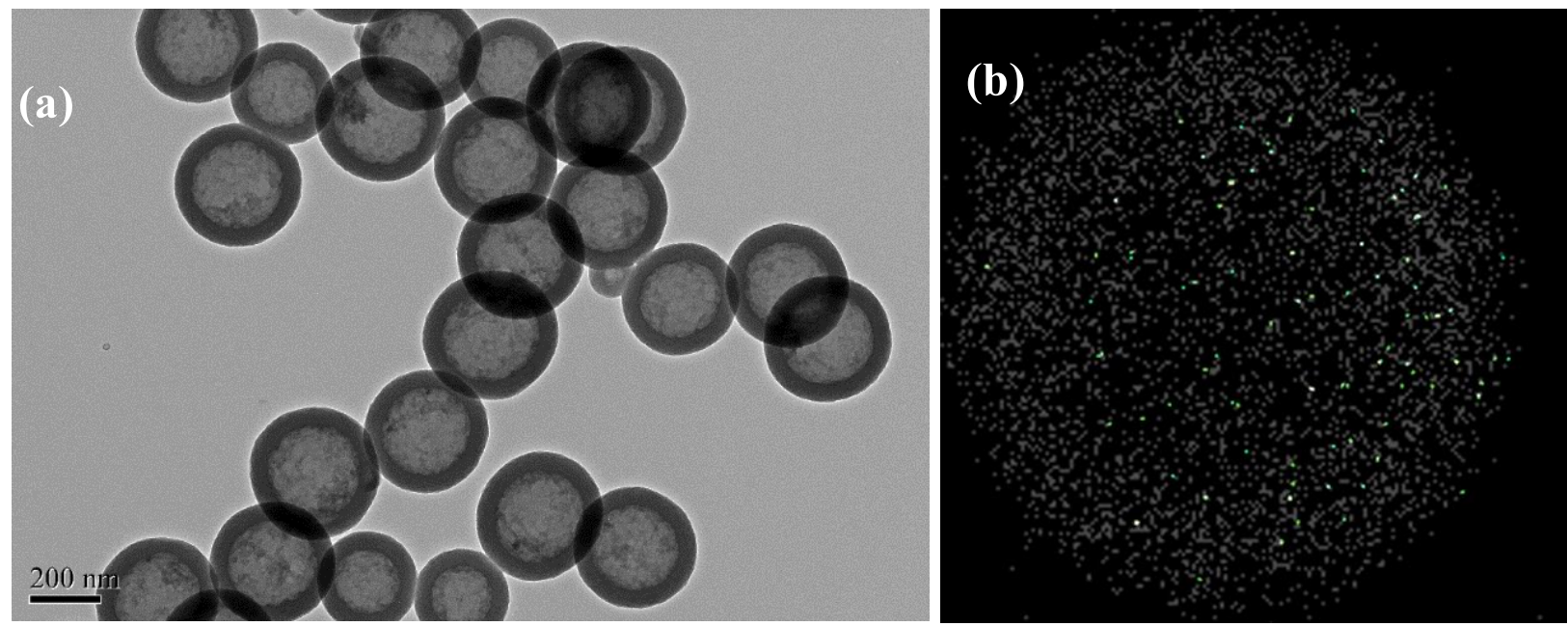

Figure S4. (a) TEM images and (b) TEM image with a chemical mapping of 3 showing the distribution of $\mathrm{Si}$ (white) and F (green).
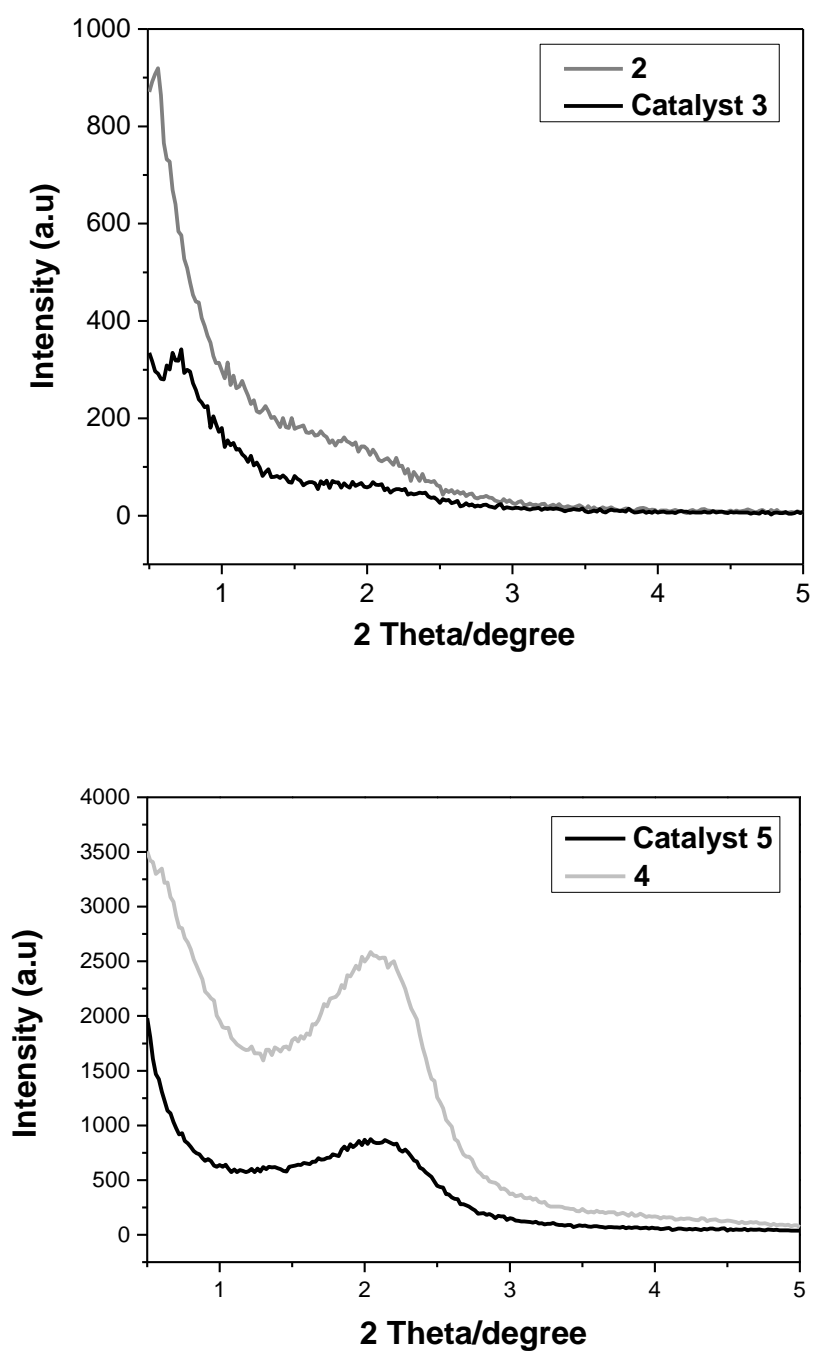

Figure S5. Small-angle powder XRD patterns of $\mathbf{2}$ and catalyst $\mathbf{3}$, and $\mathbf{4}$ and catalyst $\mathbf{5}$. 

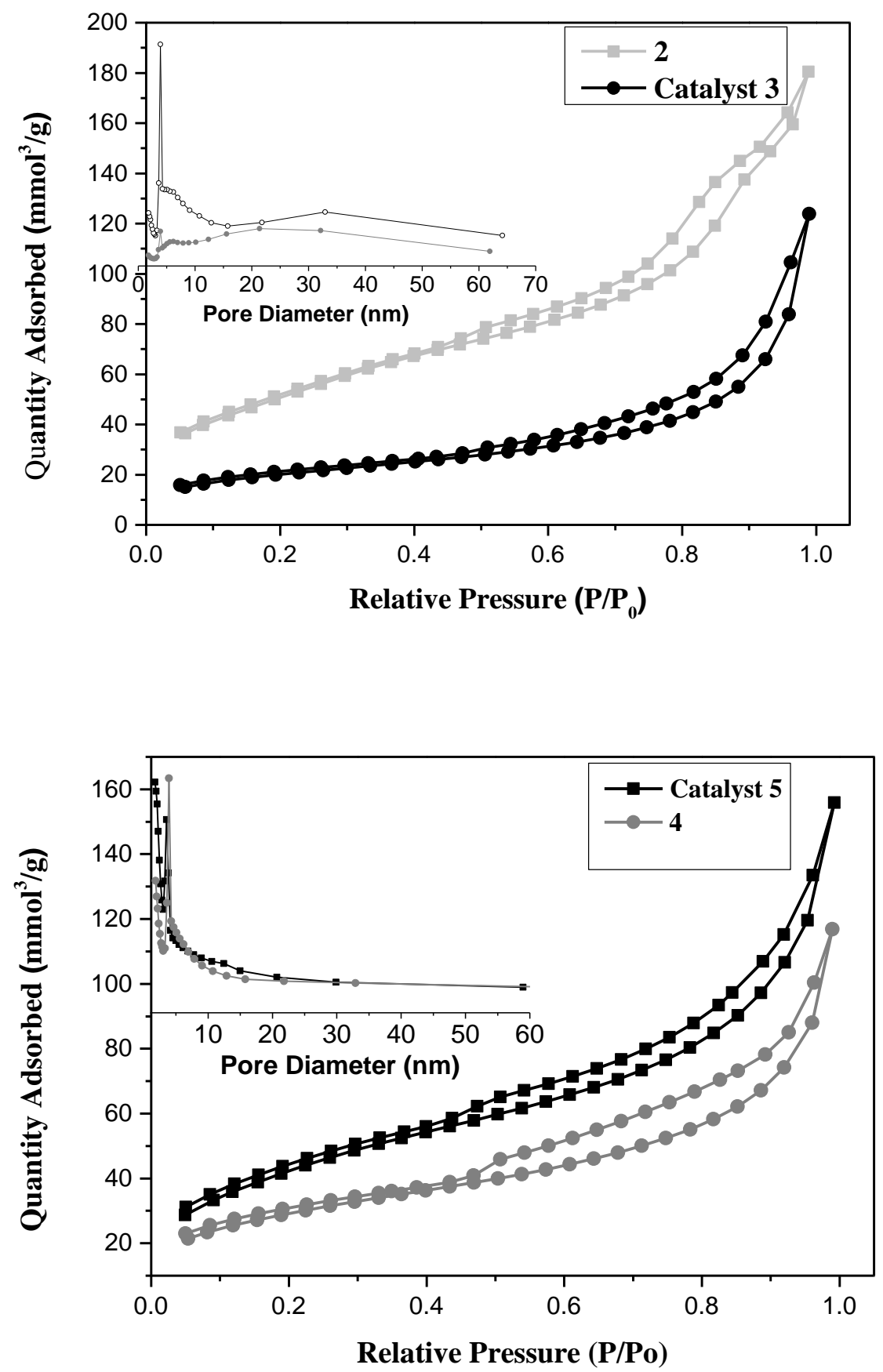

Figure S6. Nitrogen adsorption-desorption isotherms of $\mathbf{2}$ and catalyst $\mathbf{3}$, and $\mathbf{4}$ and catalyst $\mathbf{5}$. 
Results $\left(15^{\circ} \mathrm{C}\right.$ for first run $)$

Z-Average (d.nm): 407.8

Pdl: 0.197

Intercept: 0.904

Result quality Good
Size (d.n... \% Intensity Width (d.n...

$\begin{array}{llll}\text { Peak 1: } & 436.9 & 98.0 & 156.9\end{array}$

Peak 2: $5090 \quad 2.0 \quad 553.2$

$\begin{array}{llll}\text { Peak 3: } & 0.000 & 0.0 & 0.000\end{array}$

Size Distribution by Intensity

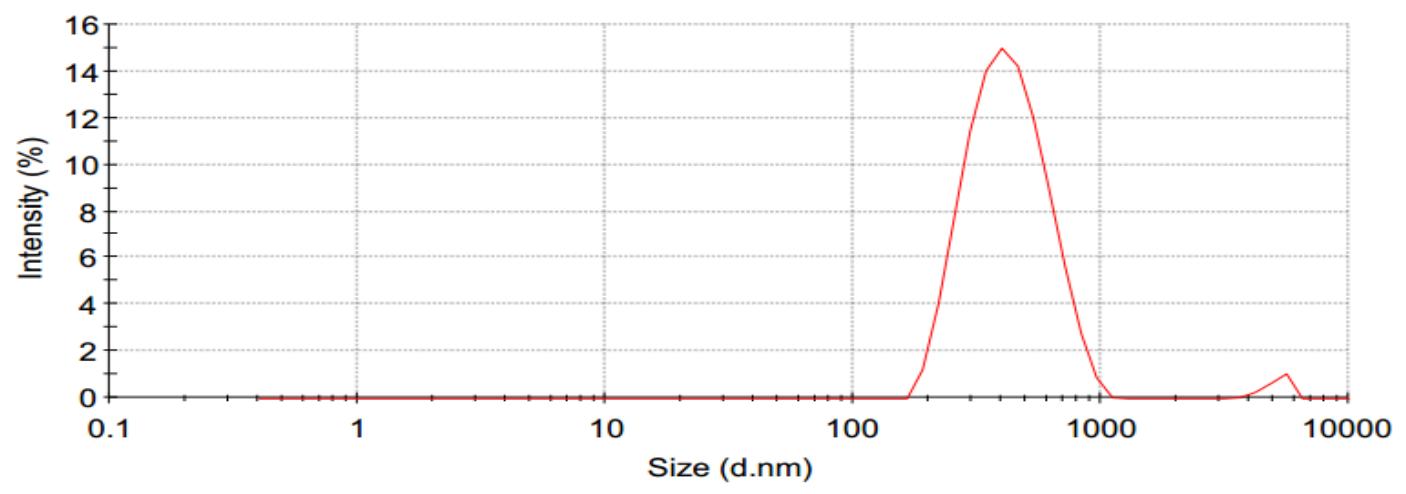

Results $\left(35^{\circ} \mathrm{C}\right.$ for first run )

$\begin{array}{rlllll} & & & \text { Size (d.n... } & \text { \% Intensity } & \text { Width (d.n... } \\ \text { Z-Average (d.nm): } & 544.8 & \text { Peak 1: } & 563.0 & 100.0 & 140.1 \\ \text { Pdl: } & 0.140 & \text { Peak 2: } & 0.000 & 0.0 & 0.000 \\ \text { Intercept: } & 0.897 & \text { Peak 3: } & 0.000 & 0.0 & 0.000 \\ \text { Result quality } & \text { Good } & & & & \end{array}$

Size Distribution by Intensity

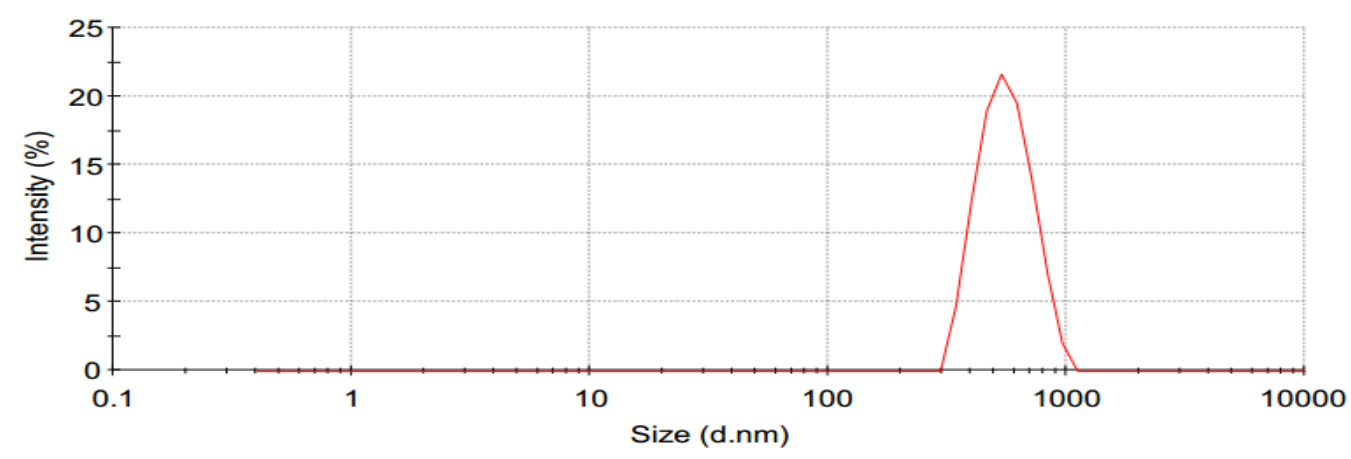




\section{Results (15 ${ }^{\circ} \mathrm{C}$ for second run)}

$\begin{array}{rlllll} & & & \text { Size (d.n... } & \text { \% Intensity } & \text { Width (d.n... } \\ \text { Z-Average (d.nm): } & 411.2 & \text { Peak 1: } & 434.0 & 98.9 & 138.1 \\ \text { Pdl: } & 0.185 & \text { Peak 2: } & 5292 & 1.1 & 407.8 \\ \text { Intercept: } & 0.905 & \text { Peak 3: } & 0.000 & 0.0 & 0.000\end{array}$

Result quality Good

Size Distribution by Intensity

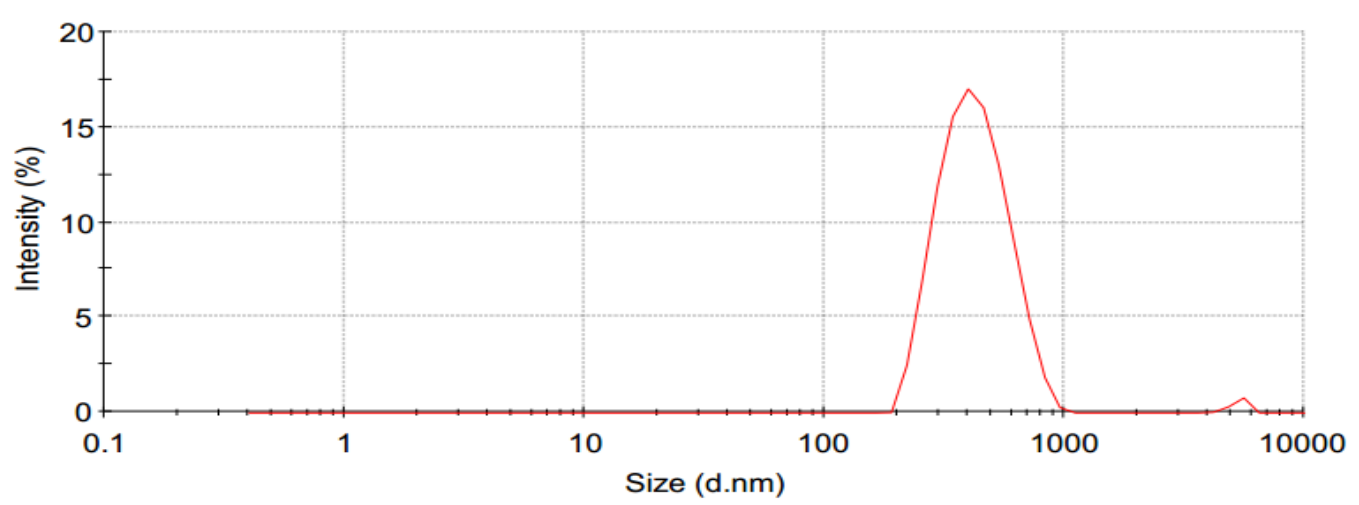

Results ( $35^{\circ} \mathrm{C}$ for second run)

Z-Average (d.nm): 553.3

$\begin{array}{rc}\text { Pdl: } & 0.172 \\ \text { Intercept: } & 0.901 \\ \text { Result quality } & \text { Good }\end{array}$

$\begin{array}{rc}\text { Pdl: } & 0.172 \\ \text { Intercept: } & 0.901 \\ \text { Result quality } & \text { Good }\end{array}$

Size (d.n... \% Intensity Width (d.n...

$\begin{array}{llll}\text { Peak 1: } & 583.8 & 100.0 & 186.4\end{array}$

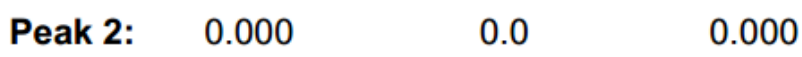

$\begin{array}{llll}\text { Peak 3: } & 0.000 & 0.0 & 0.000\end{array}$

Result quality Good

Size Distribution by Intensity

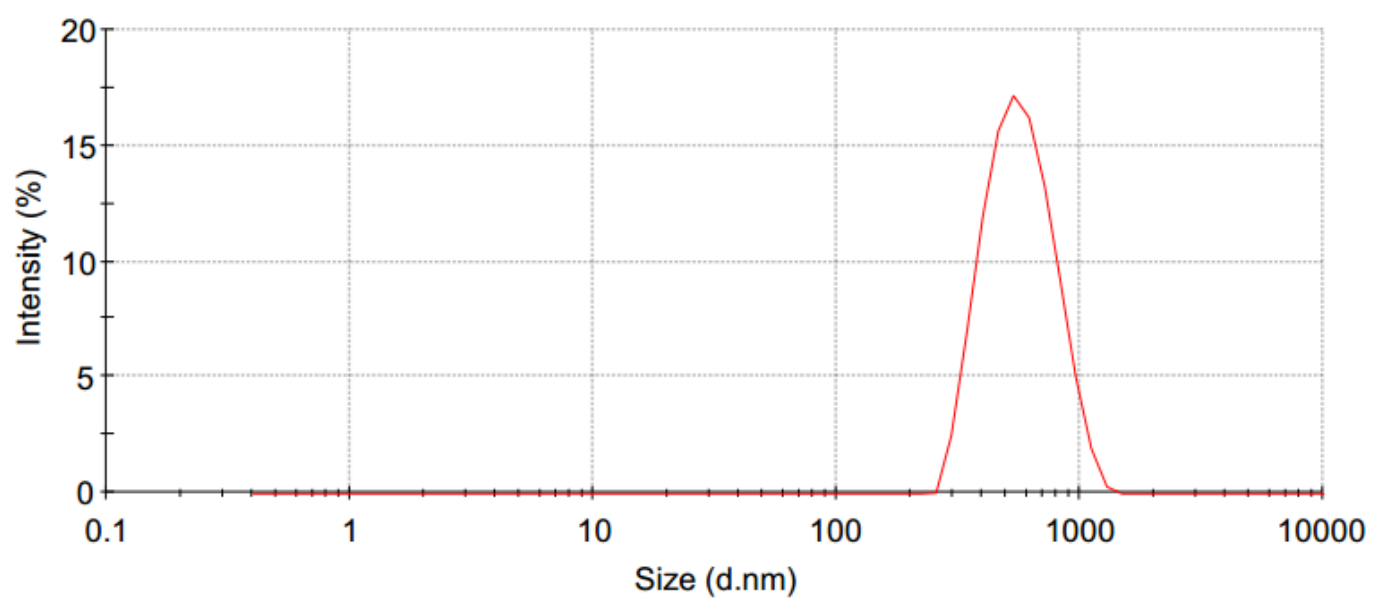




\section{Results $\left(15^{\circ} \mathrm{C}\right.$ for third run)}

Z-Average (d.nm): 422.8

Pdl: 0.185

Intercept: 0.903

Result quality Good

Size (d.n... \% Intensity Width (d.n...

$\begin{array}{llll}\text { Peak 1: } & 454.8 & 99.0 & 167.8\end{array}$

$\begin{array}{llll}\text { Peak 2: } & 5341 & 1.0 & 360.7\end{array}$

$\begin{array}{llll}\text { Peak 3: } & 0.000 & 0.0 & 0.000\end{array}$

Size Distribution by Intensity

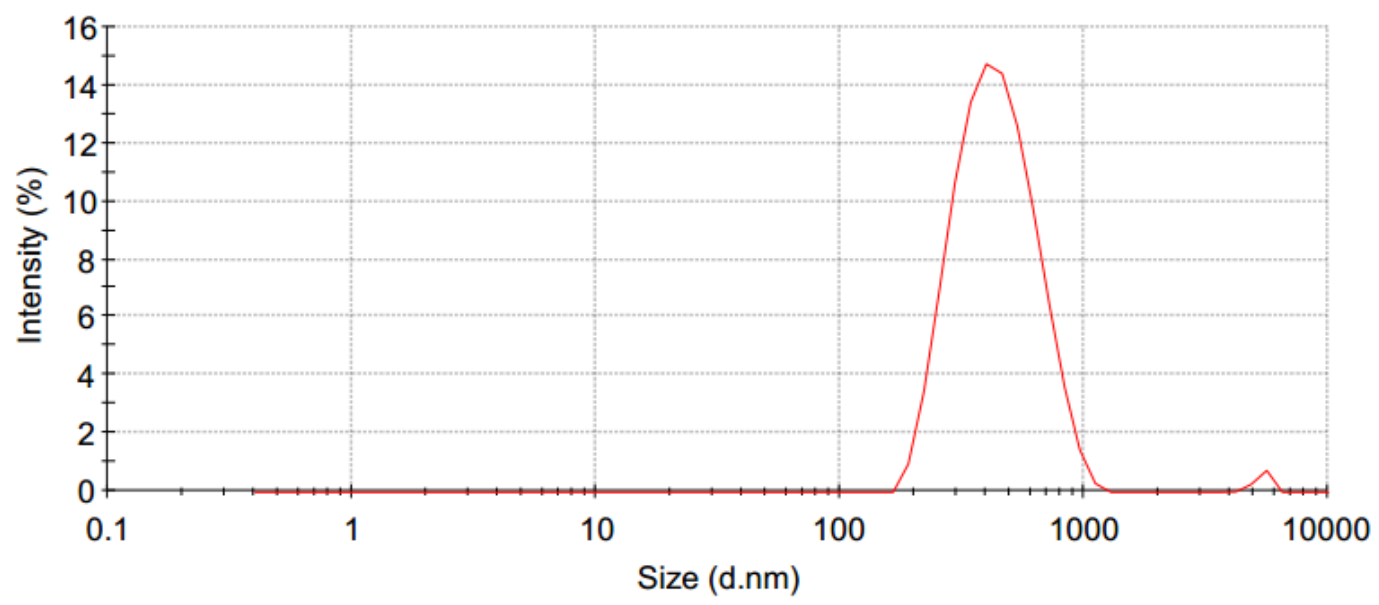

Results $\left(35^{\circ} \mathrm{C}\right.$ for third run)

$\begin{array}{rlllll} & & & \text { Size (d.n... } & \text { \% Intensity } & \text { Width (d.n... } \\ \text { Z-Average (d.nm): } & 558.9 & \text { Peak 1: } & 527.1 & 100.0 & 118.8 \\ \text { Pdl: } & 0.209 & \text { Peak 2: } & 0.000 & 0.0 & 0.000 \\ \text { Intercept: } & 0.897 & \text { Peak 3: } & 0.000 & 0.0 & 0.000 \\ \text { Result quality } & \text { Good } & & & & \end{array}$

Size Distribution by Intensity

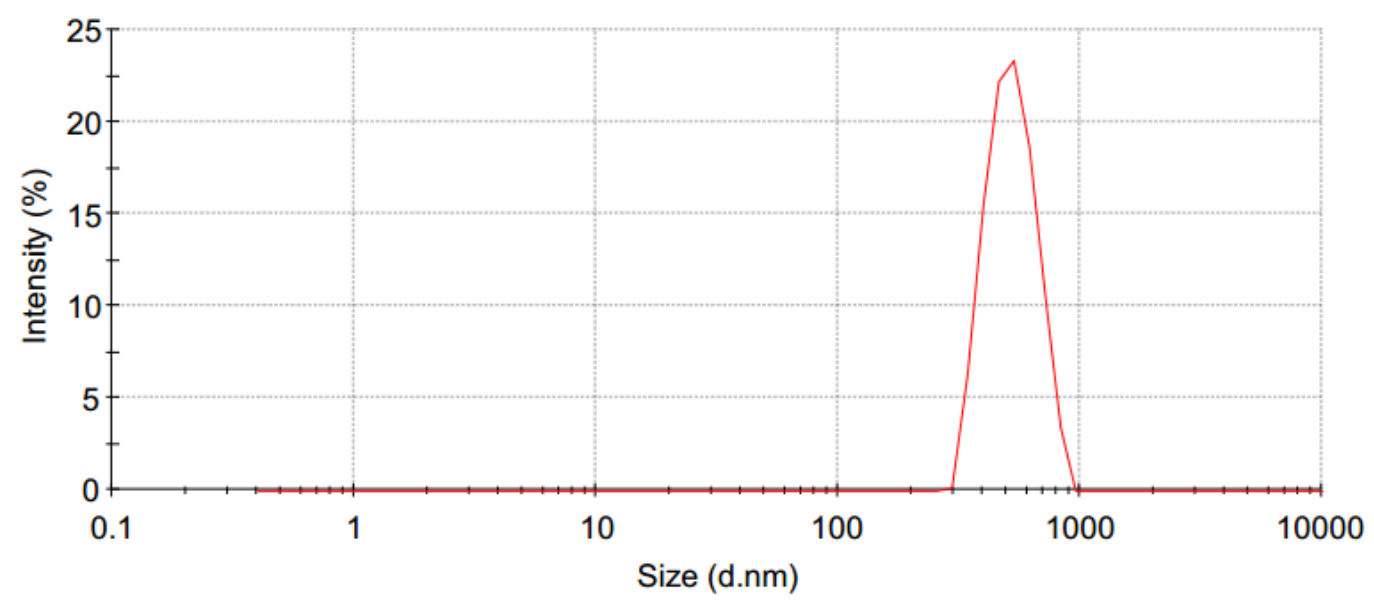


Results ( $15^{\circ} \mathrm{C}$ for fourth run)

$\begin{array}{rlllll} & & & \text { Size (d.n... } & \text { \% Intensity } & \text { Width (d.n... } \\ \text { Z-Average (d.nm): } & 434.6 & \text { Peak 1: } & 469.5 & 100.0 & 160.3 \\ \text { Pdl: } & 0.160 & \text { Peak 2: } & 0.000 & 0.0 & 0.000 \\ \text { Intercept: } & 0.899 & \text { Peak 3: } & 0.000 & 0.0 & 0.000\end{array}$

Result quality Good

Size Distribution by Intensity

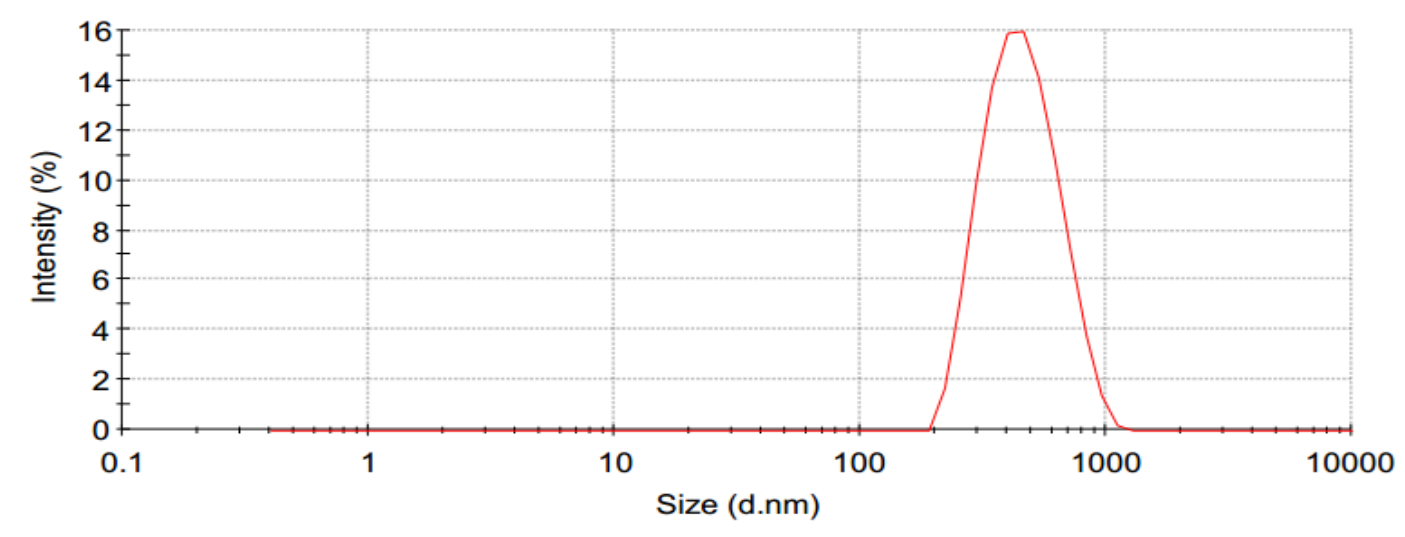

\section{Results ( $35{ }^{\circ} \mathrm{C}$ for fourth run)}

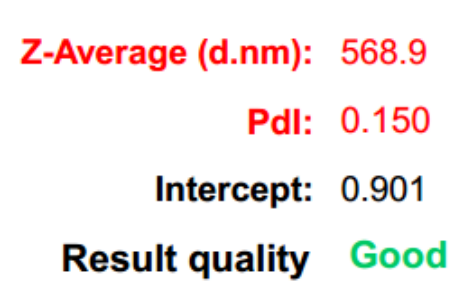

Size (d.n... \% Intensity Width (d.n...

$\begin{array}{lll}\text { Peak 1: } & 572.1 & 100.0\end{array}$

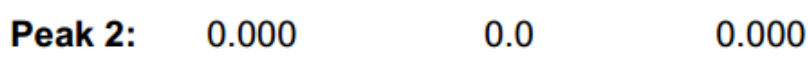

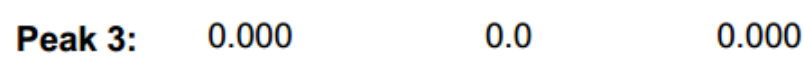

Result quality Good

Size Distribution by Intensity

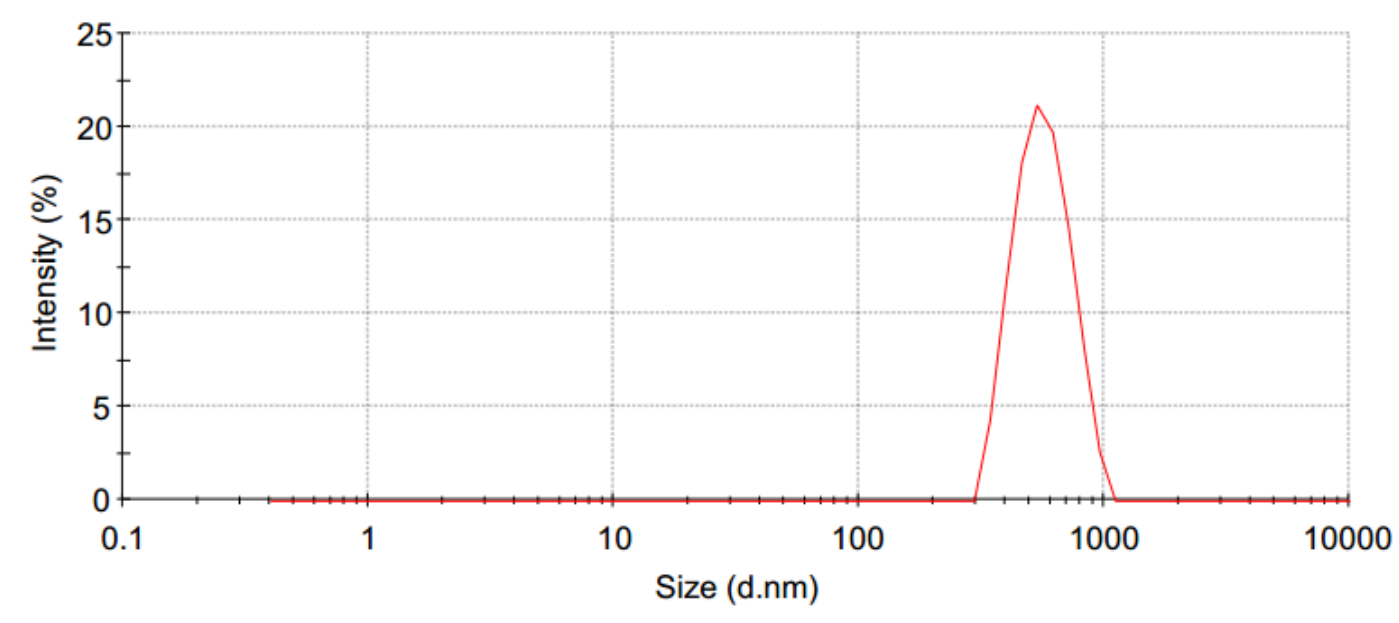


Results $\left(15^{\circ} \mathrm{C}\right.$ for fifth run)

$\begin{array}{rlllll} & & & \text { Size (d.n... } & \% \text { Intensity } & \text { Width (d.n... } \\ \text { Z-Averaqe (d.nm): } & 440.8 & \text { Peak 1: } & 517.6 & 100.0 & 210.6 \\ \text { Pdl: } & 0.139 & \text { Peak 2: } & 0.000 & 0.0 & 0.000 \\ \text { Intercept: } & 0.907 & \text { Peak 3: } & 0.000 & 0.0 & 0.000 \\ \text { Result quality } & \text { Good } & & & & \end{array}$

Size Distribution by Intensity

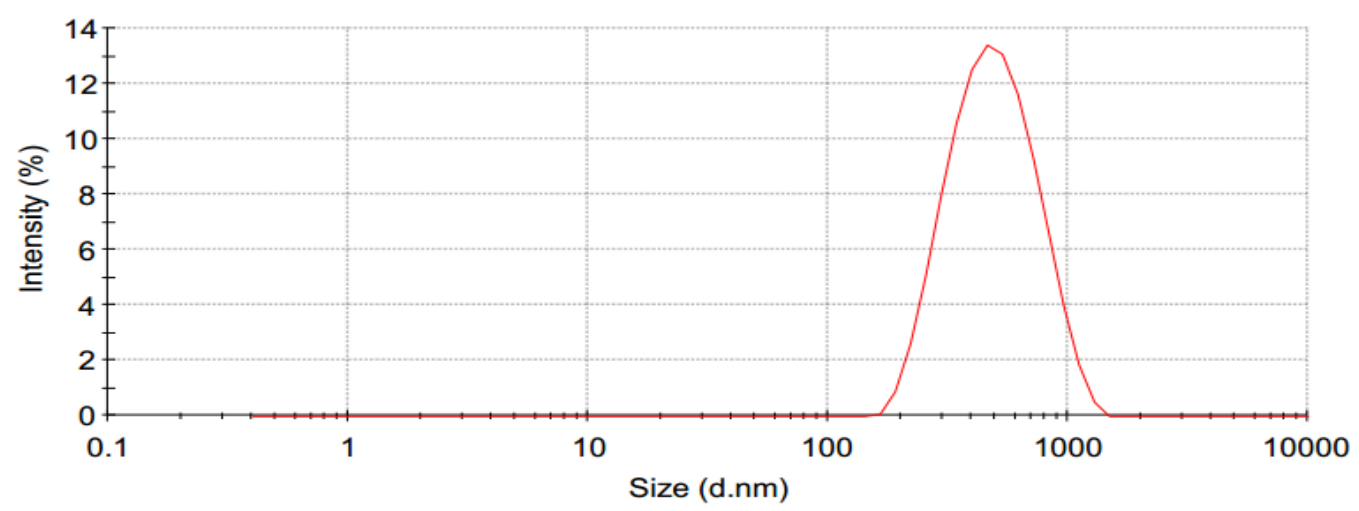

Results $\left(35^{\circ} \mathrm{C}\right.$ for fifth run)

$\begin{array}{rlllll} & & & \text { Size (d.n... } & \text { \% Intensity } & \text { Width (d.n... } \\ \text { Z-Average (d.nm): } & 571.3 & \text { Peak 1: } & 650.8 & 100.0 & 245.6 \\ \text { Pdl: } & 0.115 & \text { Peak 2: } & 0.000 & 0.0 & 0.000 \\ \text { Intercept: } & 0.903 & \text { Peak 3: } & 0.000 & 0.0 & 0.000 \\ \text { Result quality } & \text { Good } & & & & \end{array}$

Size Distribution by Intensity

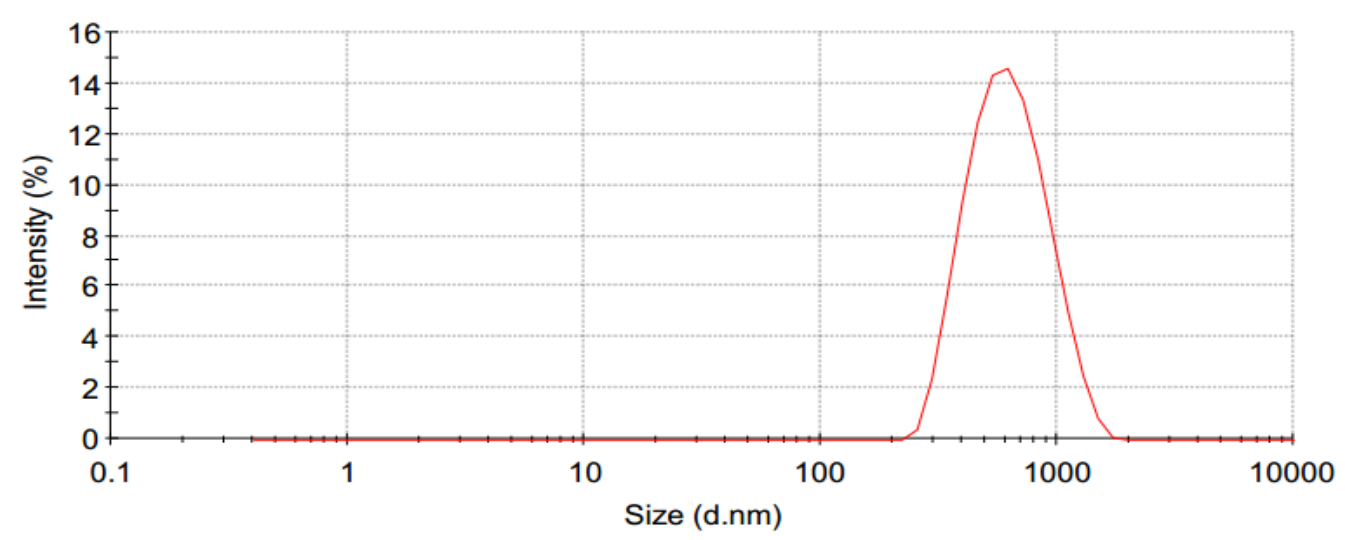

Figure S7. Average hydrodynamic diameters distribution measurement of the recycled catalyst 5. 


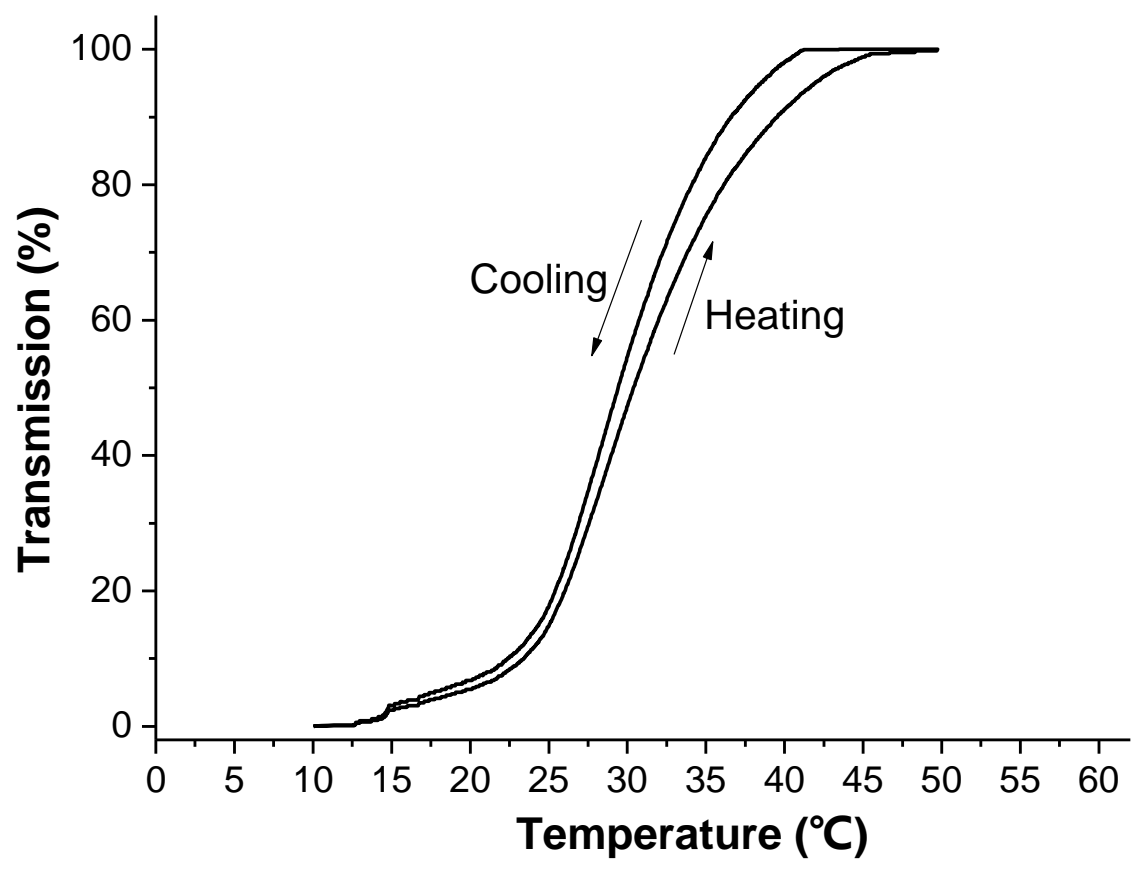

Figure S8. The turbidity curves of the polymer-coating 4' (This turbidity measurement was performed on a custom-modified Tepper turbidity photometer TP1-D at a wavelength of $670 \mathrm{~nm}$, a cell path length of $10 \mathrm{~mm}$, and magnetic stirring. The heating program started at high temperature, and it was cooled to $10{ }^{\circ} \mathrm{C}$ at a constant cooling rate of $1.0{ }^{\circ} \mathrm{C} / \mathrm{min}$ followed by heating back to the starting temperature at the same rate). 
Table S1. Optimizing reaction conditions for the Michael addition reaction. ${ }^{\text {a }}$

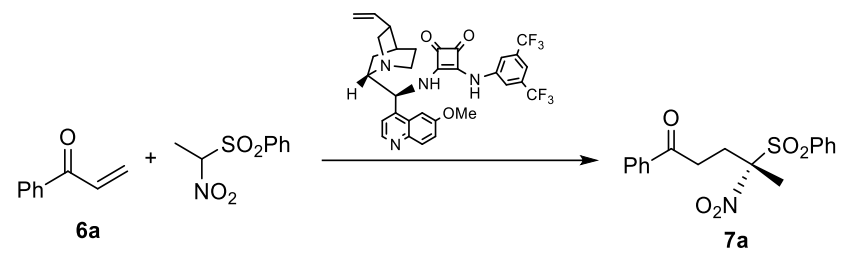

\begin{tabular}{lllllll}
\hline Entry & Solvent & Additive & ${ }^{\circ} \mathrm{C}$ & Time $(\mathrm{h})$ & $\begin{array}{l}\text { Yield } \\
(\%)\end{array}$ & ee(\%) \\
\hline 1 & Toluene & $/$ & -35 & 8 & 98 & 97 \\
1 & Toluene $/ \mathrm{H}_{2} \mathrm{O}(3: 1)$ & $/$ & 0 & 4 & 98 & 90 \\
2 & Toluene $/ \mathrm{H}_{2} \mathrm{O}(2: 1)$ & $/$ & 0 & 4 & 98 & 92 \\
3 & Toluene $/ \mathrm{H}_{2} \mathrm{O}(1: 1)$ & $/$ & 0 & 4 & 98 & 95 \\
4 & Toluene $/ \mathrm{H}_{2} \mathrm{O}(1: 1)$ & $/$ & -15 & 8 & 98 & 96 \\
5 & Toluene $/ \mathrm{H}_{2} \mathrm{O}(1: 1)$ & $/$ & -35 & 8 & 98 & 97 \\
6 & Toluene $/ \mathrm{H}_{2} \mathrm{O}(1: 1)$ & $/$ & -40 & 8 & 95 & 97 \\
$7^{b}$ & Toluene $/ \mathrm{H}_{2} \mathrm{O}(1: 1)$ & $\mathrm{HCOONa}$ & -35 & 6 & 98 & 97 \\
\hline
\end{tabular}

${ }^{a}$ Reaction conditions: Chiral squaramide (0.5 mol\%), 1-phenylprop-2-en-1-one (0.10 mmol), ((1nitroethyl)sulfonyl)benzene $(0.12 \mathrm{mmol})$, and $2.0 \mathrm{~mL}$ of solvent. ${ }^{\mathrm{b}}$ Data was obtained in the presence of 10 equivalent of HCOONa."

Table S2. Optimizing reaction conditions for the asymmetric hydrogenation. ${ }^{a}$

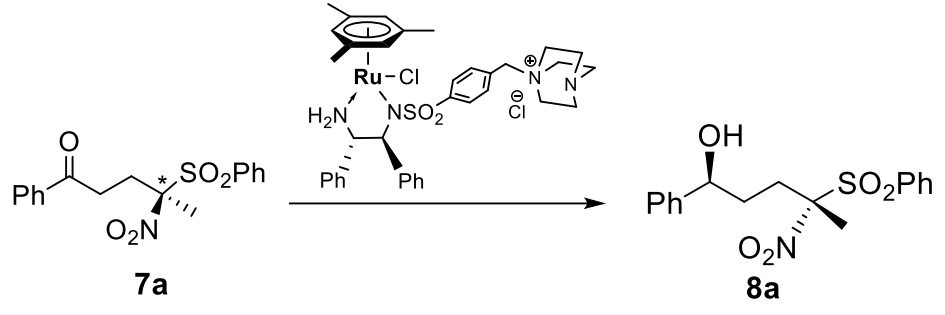

\begin{tabular}{lllllll}
\hline Entry & H-resource, Solvent & ${ }^{\mathbf{0}} \mathbf{C}$ & Time (h) & Yield (\%) & $\boldsymbol{e e ~ ( \% ) ~}$ & $\boldsymbol{d r}(\%)$ \\
\hline 1 & $\mathrm{HCO}_{2} \mathrm{Na}, \mathrm{H}_{2} \mathrm{O}(1: 1)$ & 35 & 18 & 83 & 99 & $98: 2$ \\
2 & $\mathrm{HCO}_{2} \mathrm{Na}$, Toluene/ $\mathrm{H}_{2} \mathrm{O}(3: 1)$ & 35 & 12 & 91 & 92 & $94: 6$ \\
3 & $\mathrm{HCO}_{2} \mathrm{Na}$, Toluene/ $\mathrm{H}_{2} \mathrm{O}(2: 1)$ & 35 & 12 & 93 & 96 & $96: 4$ \\
3 & $\mathrm{HCO}_{2} \mathrm{Na}$, Toluene/ $\mathrm{H}_{2} \mathrm{O}(1: 1)$ & 35 & 12 & 96 & 99 & $98: 2$ \\
4 & $\mathrm{HCO}_{2} \mathrm{Na}$, Toluene/ $\mathrm{H}_{2} \mathrm{O}(1: 1)$ & 30 & 12 & 89 & 90 & $98: 2$ \\
5 & $\mathrm{HCO}_{2} \mathrm{Na}$, Toluene/ $\mathrm{H}_{2} \mathrm{O}(1: 1)$ & 40 & 10 & 96 & 97 & $96: 4$ \\
\hline
\end{tabular}

${ }^{a}$ Reaction conditions: Chiral MestyleneRuArDPEN (1.0 mol\%), H-resource (1.0 mmol), (S)-4-nitro1-phenyl-4-(phenylsulfonyl)pentan-1-one (97\%ee) (0.10 mmol), and $2.0 \mathrm{~mL}$ solvent. 
8a: (1S,4S)-4-nitro-1-phenyl-4-(phenylsulfonyl)pentan-1-ol: (HPLC: Chiracel OZ-H, detected at $254 \mathrm{~nm}$, eluent: $\mathrm{n}$-hexane $/ 2$-propanol $=90 / 10$, flow rate $=1.0 \mathrm{~mL} / \mathrm{min}, 25^{\circ} \mathrm{C}$ ).
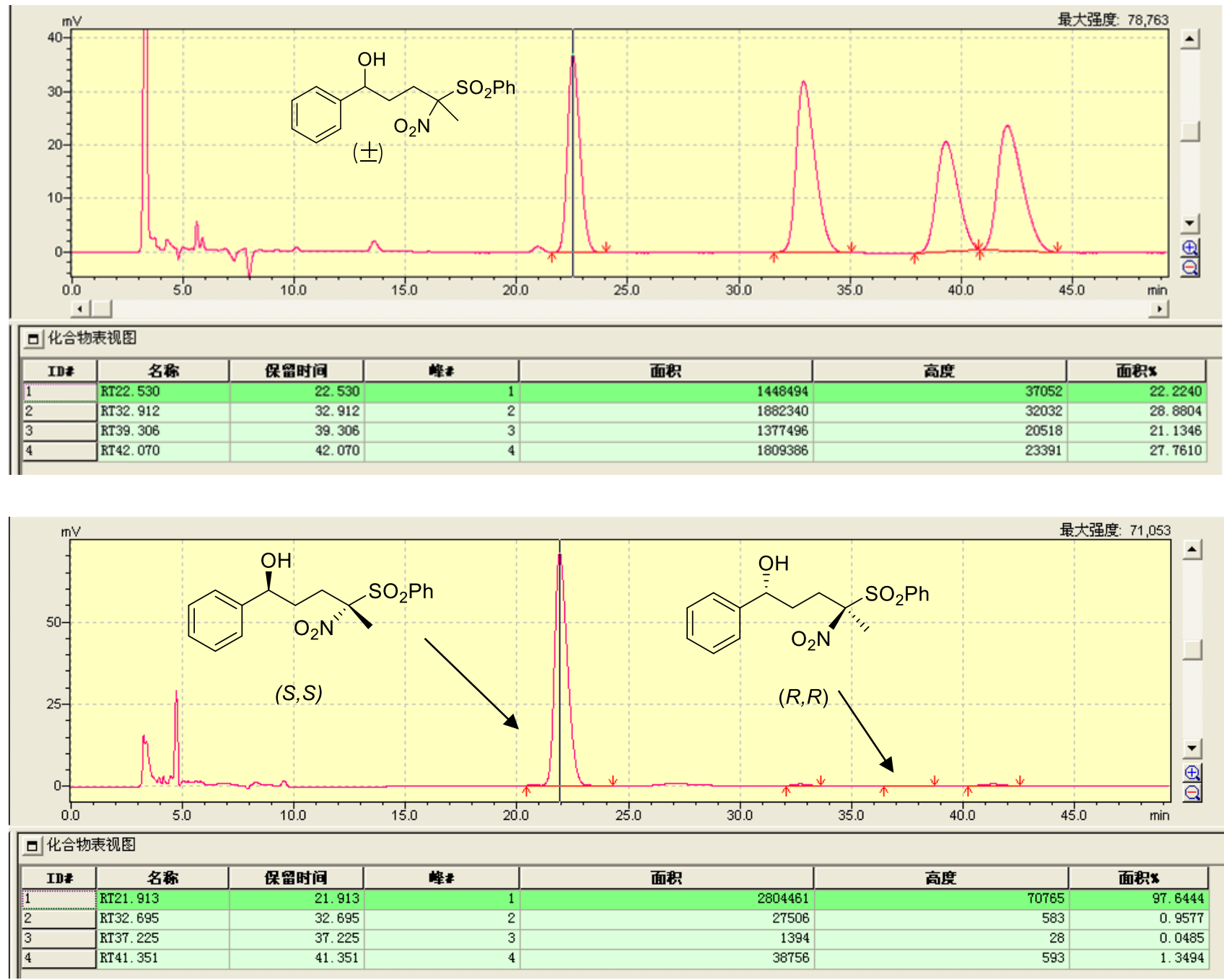

Translation of all characters (Chinese) in above two frameworks to English is as follows:

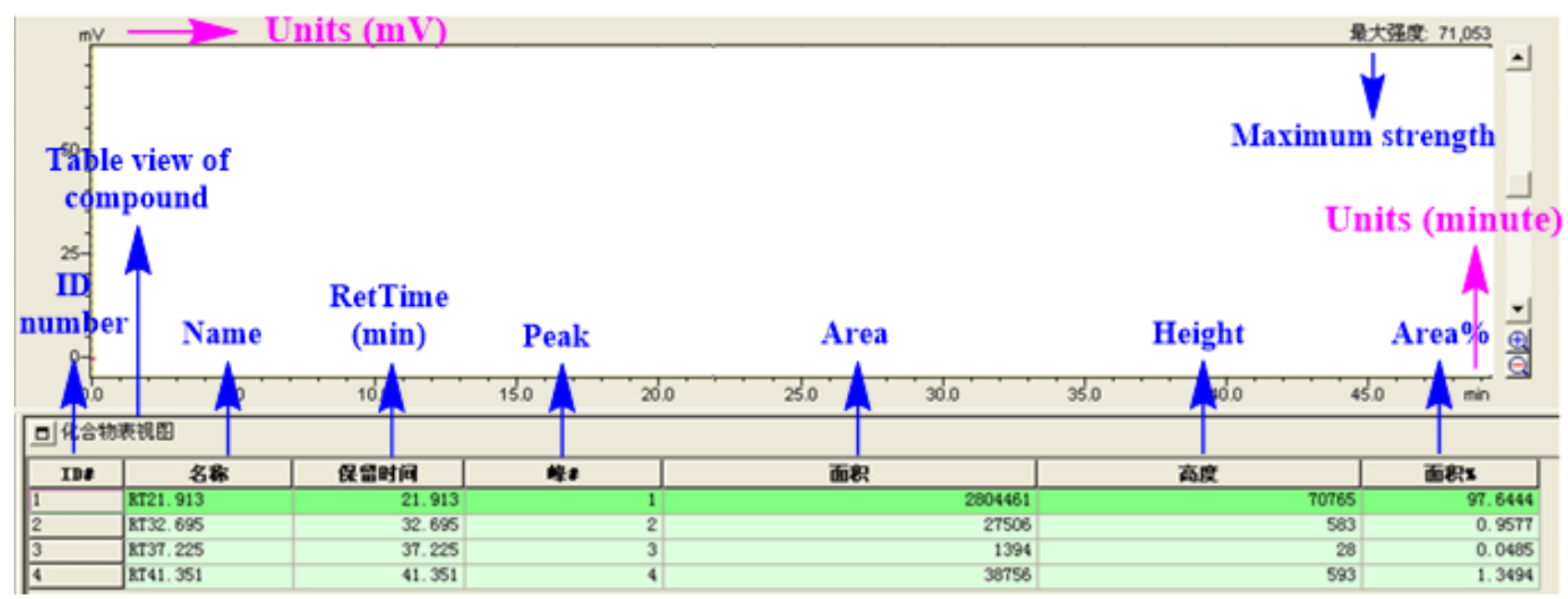


8b: (1S,4S)-1-(3-fluorophenyl)-4-nitro-4-(phenylsulfonyl)pentan-1-ol: (HPLC: Chiracel IC, detected at $254 \mathrm{~nm}$, eluent: $\mathrm{n}$-hexane $/ 2$-propanol $=85 / 15$, flow rate $=1.0 \mathrm{~mL} / \mathrm{min}, 25^{\circ} \mathrm{C}$ ).
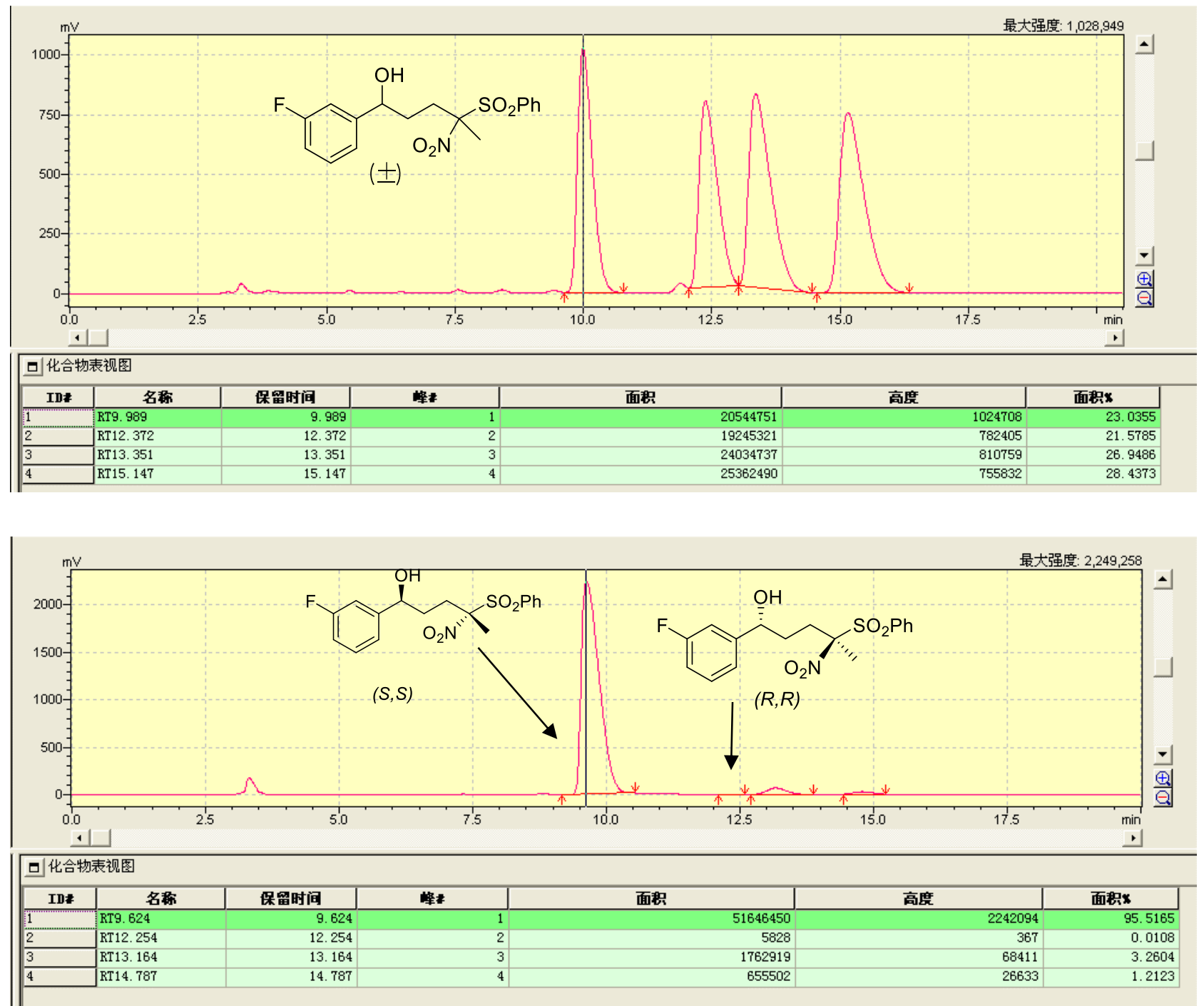

Translation of all characters (Chinese) in above two frameworks to English is as follows:

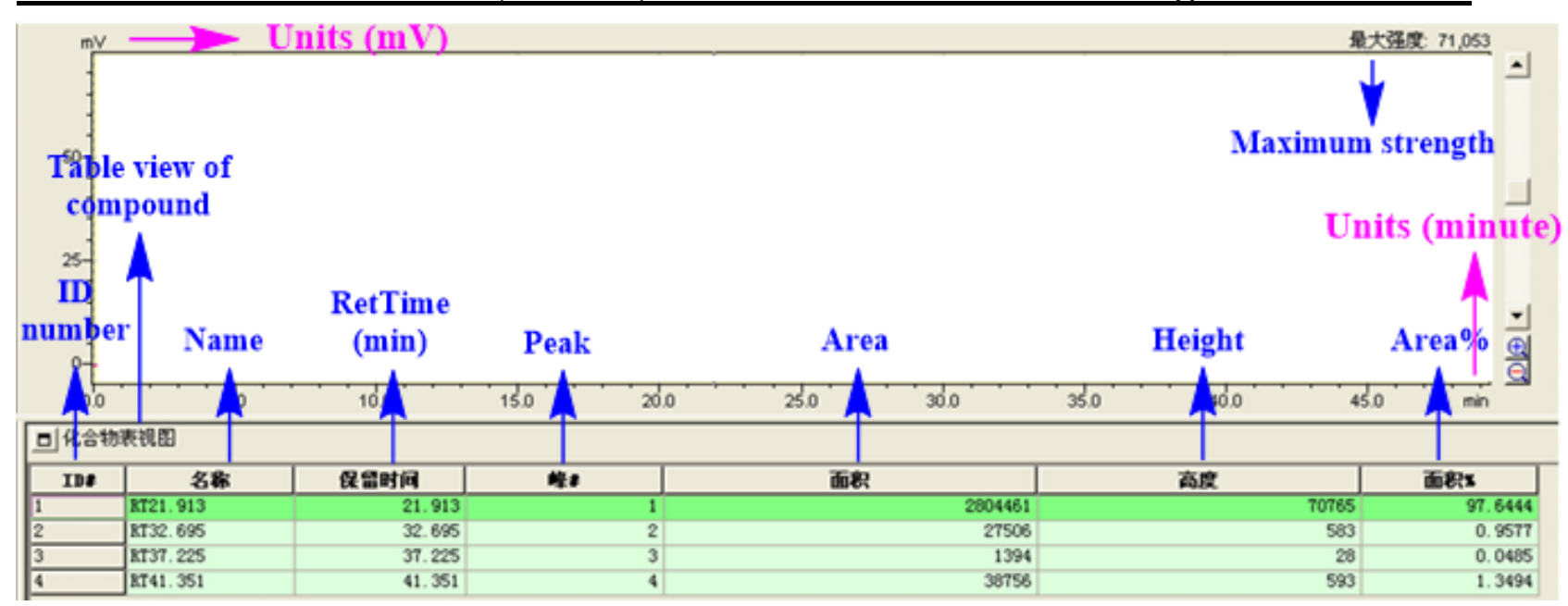


8c: (1S,4S)-1-(2-fluorophenyl)-4-nitro-4-(phenylsulfonyl)pentan-1-ol: (HPLC: Chiracel OZ-H, detected at $254 \mathrm{~nm}$, eluent: $\mathrm{n}$-hexane $/ 2$-propanol $=90 / 10$, flow rate $=0.8 \mathrm{~mL} / \mathrm{min}, 25^{\circ} \mathrm{C}$ ).
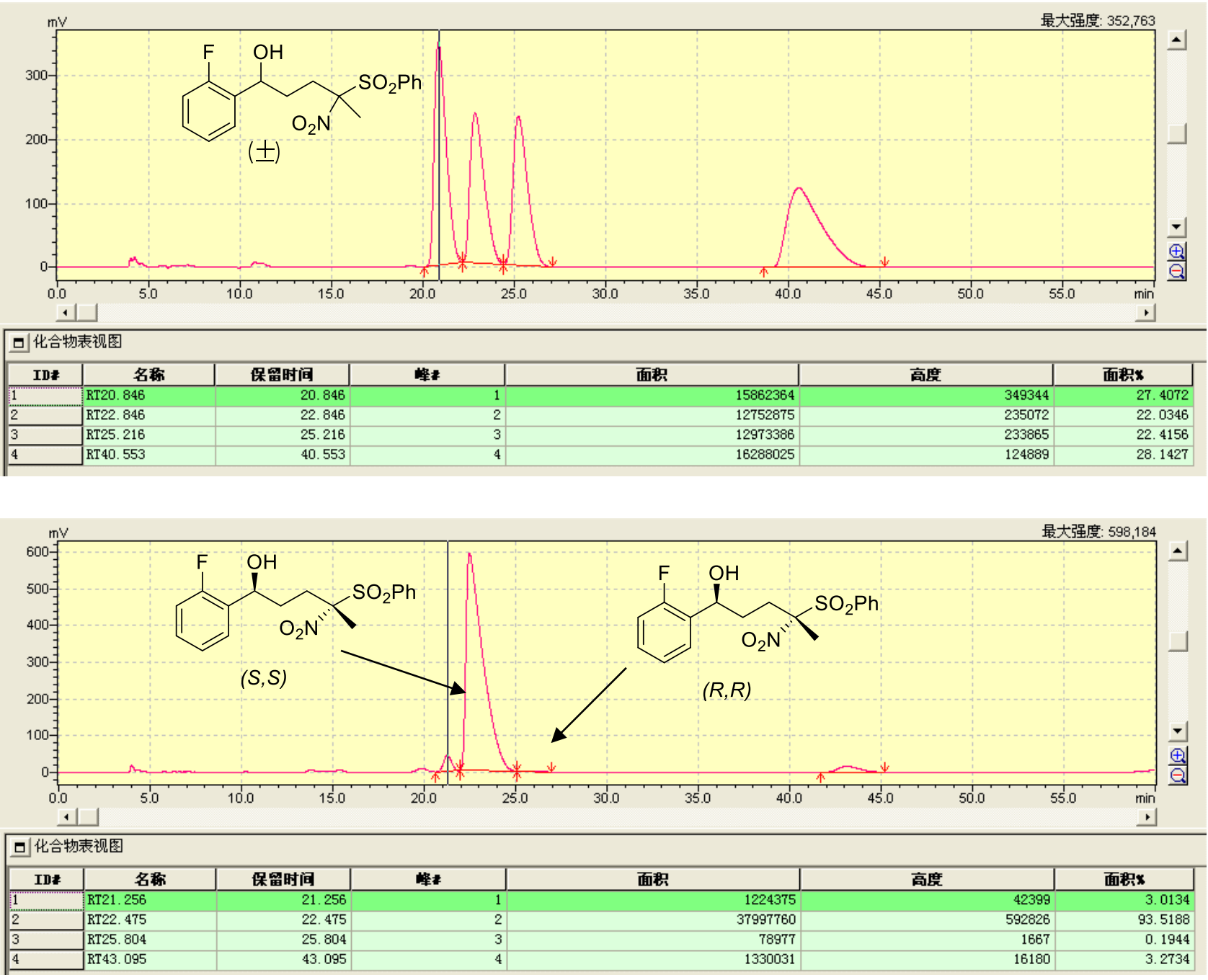

Translation of all characters (Chinese) in above two frameworks to English is as follows:

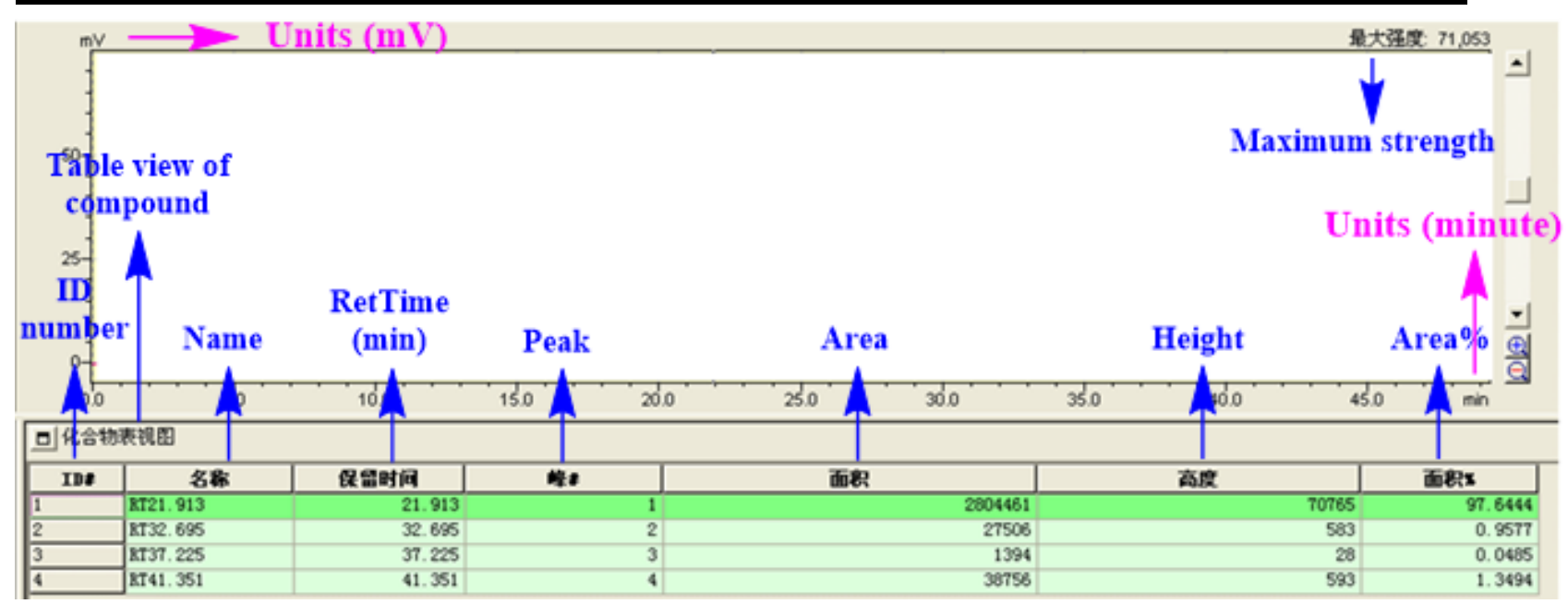


8d: (1S,4S)-1-(4-chlorophenyl)-4-nitro-4-(phenylsulfonyl)pentan-1-ol: (HPLC: Chiracel OZ-H, detected at $254 \mathrm{~nm}$, eluent: $\mathrm{n}$-hexane $/ 2$-propanol $=90 / 10$, flow rate $=1.0 \mathrm{~mL} / \mathrm{min}, 25^{\circ} \mathrm{C}$ ).
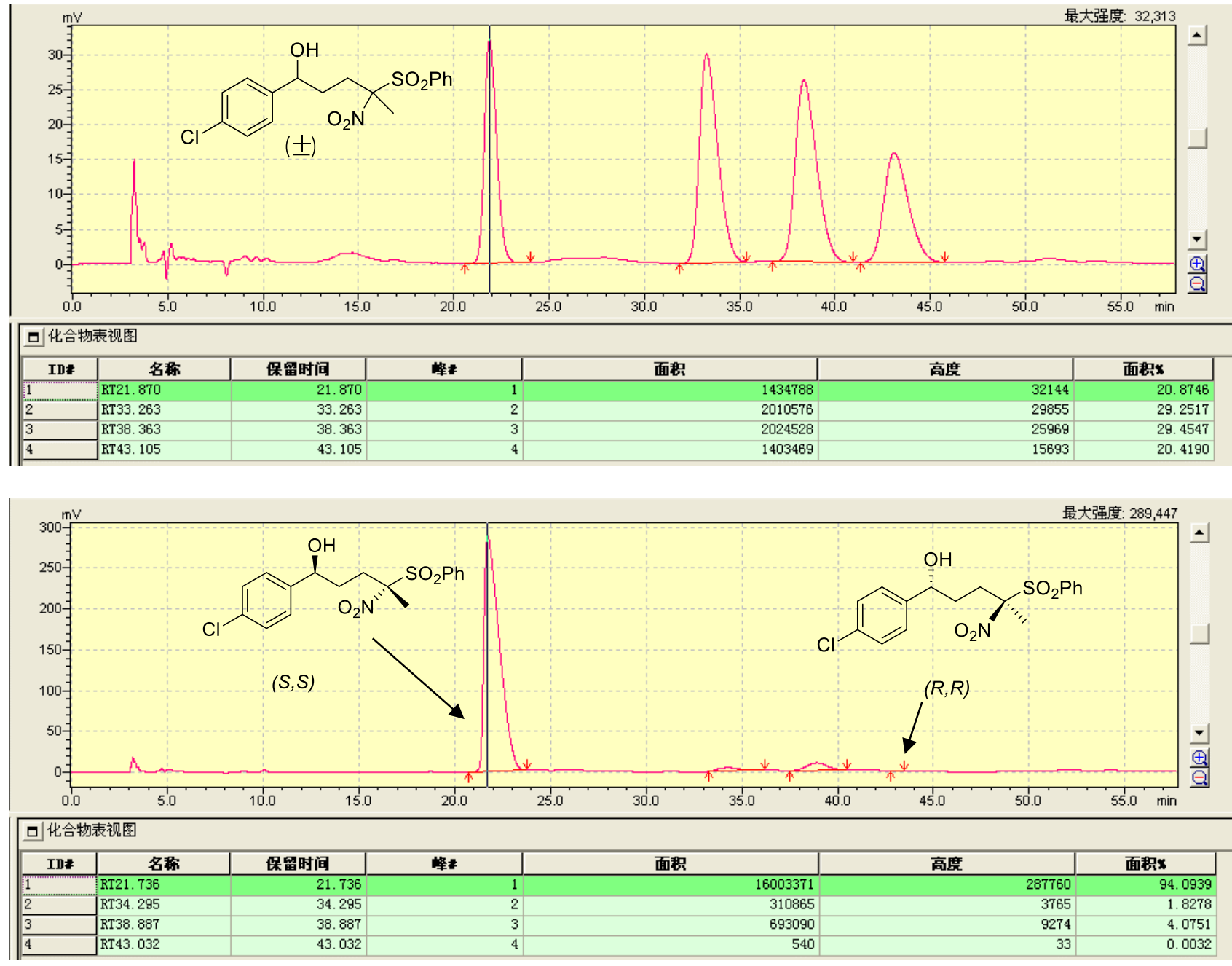

Translation of all characters (Chinese) in above two frameworks to English is as follows:

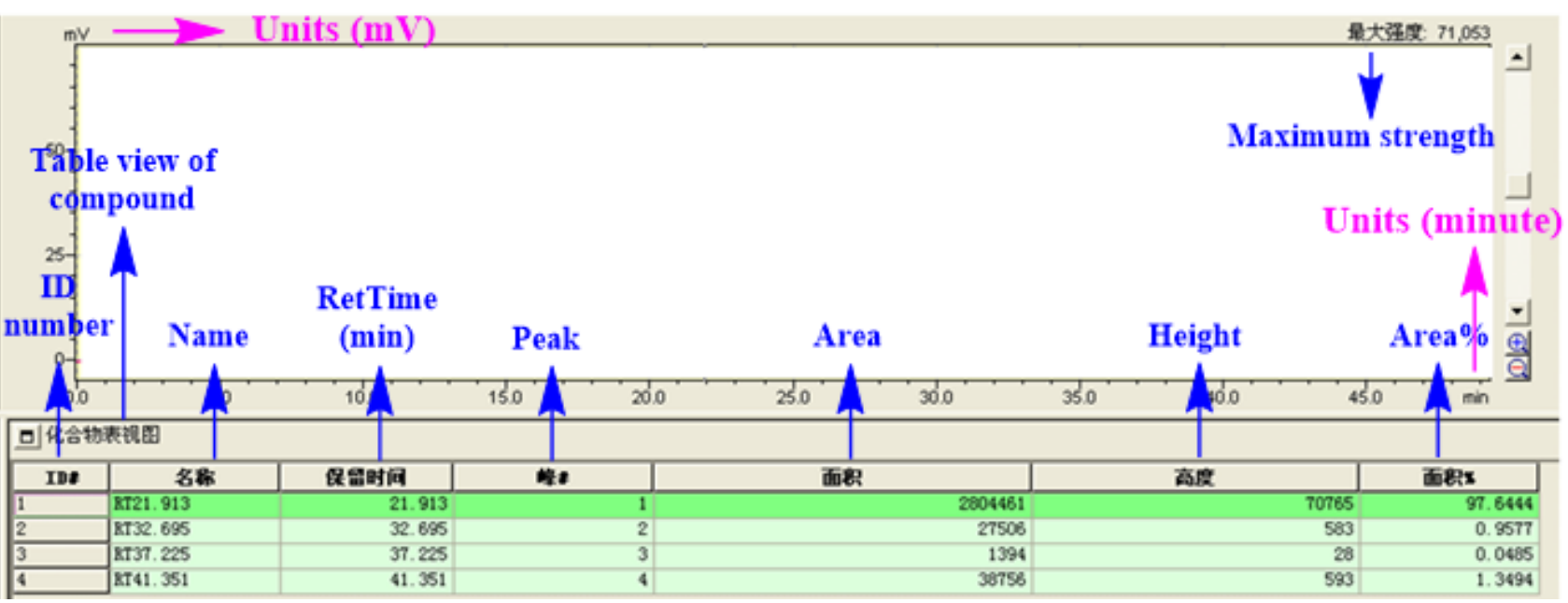


8e: (1S,4S)-1-(2-chlorophenyl)-4-nitro-4-(phenylsulfonyl)pentan-1-ol: (HPLC: Chiracel OZ-H, detected at $254 \mathrm{~nm}$, eluent: $\mathrm{n}$-hexane $/ 2$-propanol $=90 / 10$, flow rate $=0.5 \mathrm{~mL} / \mathrm{min}, 25^{\circ} \mathrm{C}$ ).
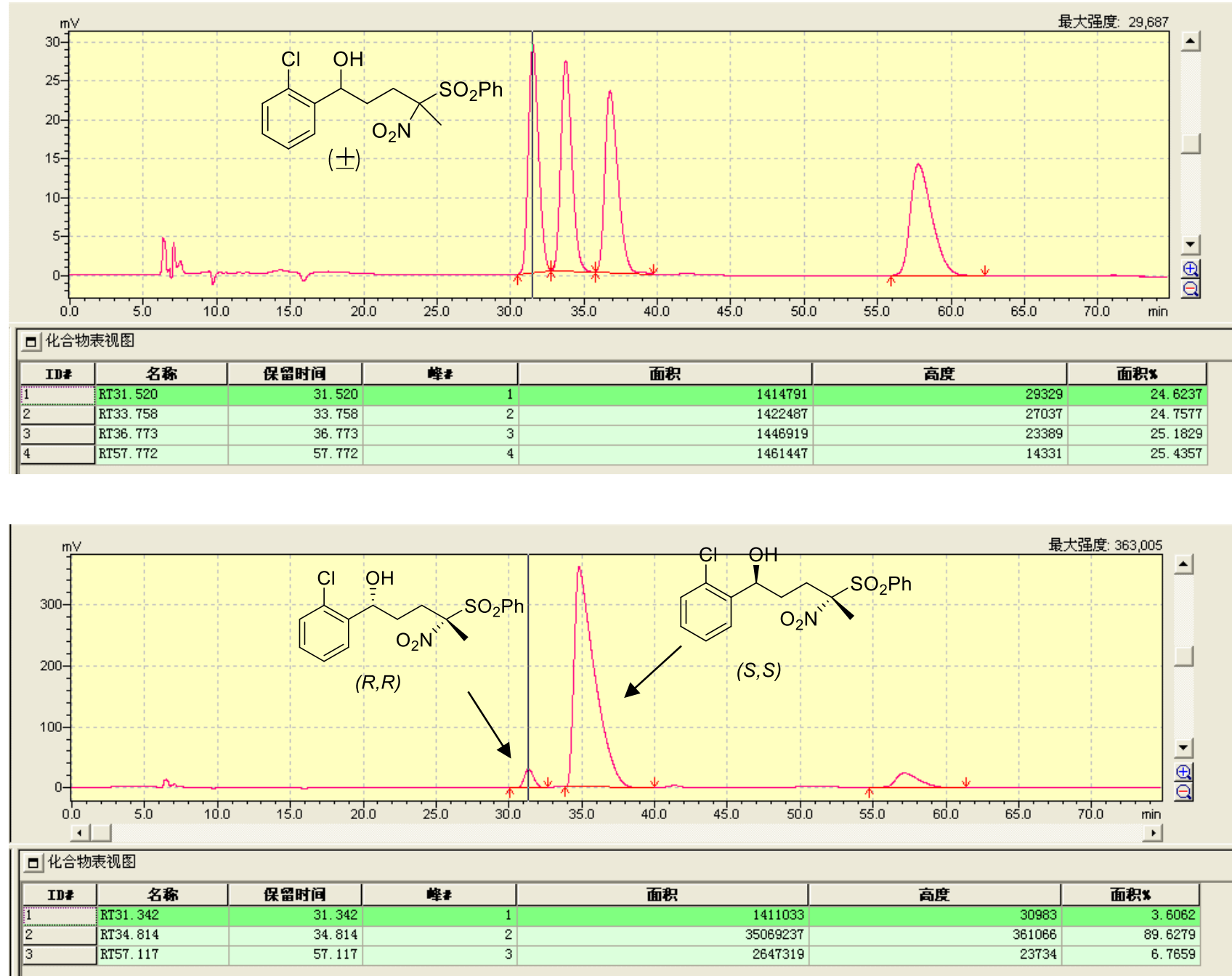

Translation of all characters (Chinese) in above two frameworks to English is as follows:

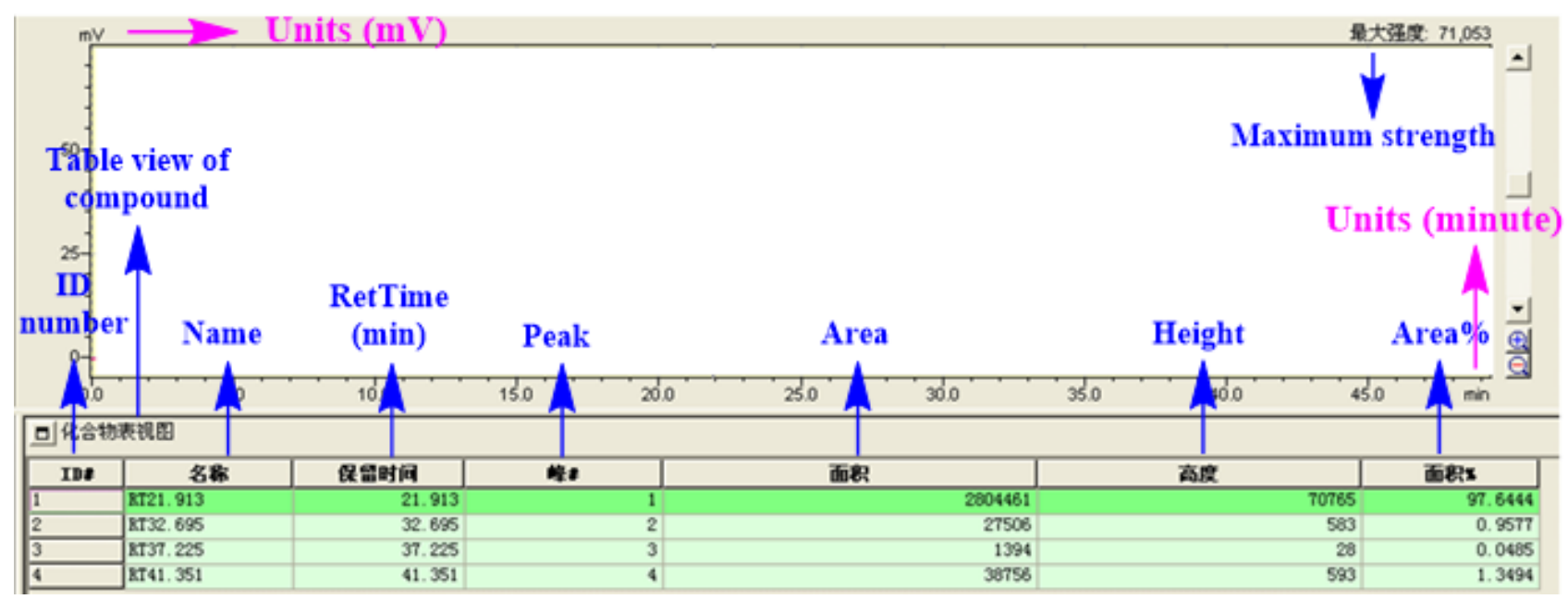


8f: (1S,4S)-1-(4-bromophenyl)-4-nitro-4-(phenylsulfonyl)pentan-1-ol: (HPLC: Chiracel OZ-H, detected at $254 \mathrm{~nm}$, eluent: $\mathrm{n}$-hexane $/ 2$-propanol $=90 / 10$, flow rate $=1.0 \mathrm{~mL} / \mathrm{min}, 25^{\circ} \mathrm{C}$ ).
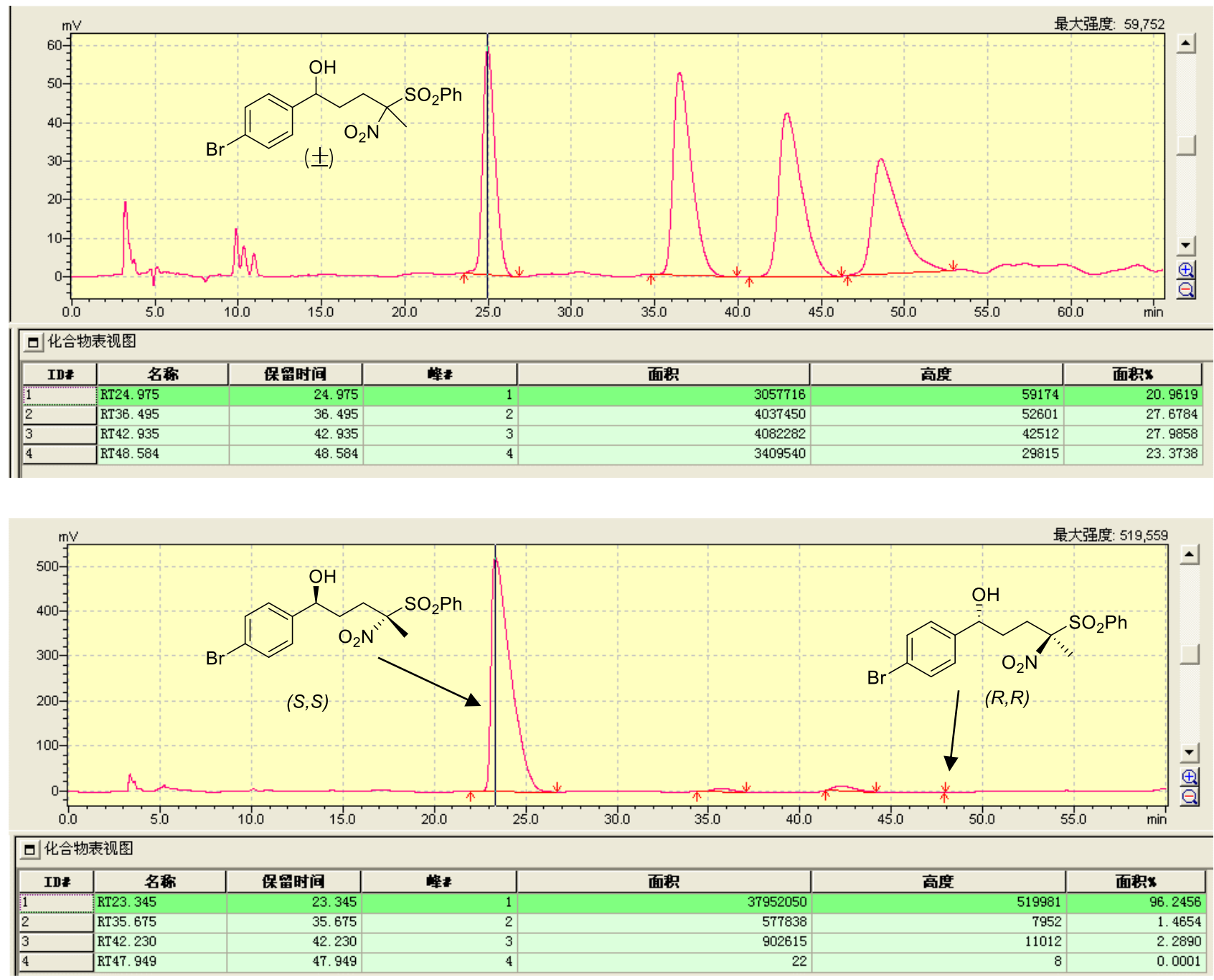

Translation of all characters (Chinese) in above two frameworks to English is as follows:

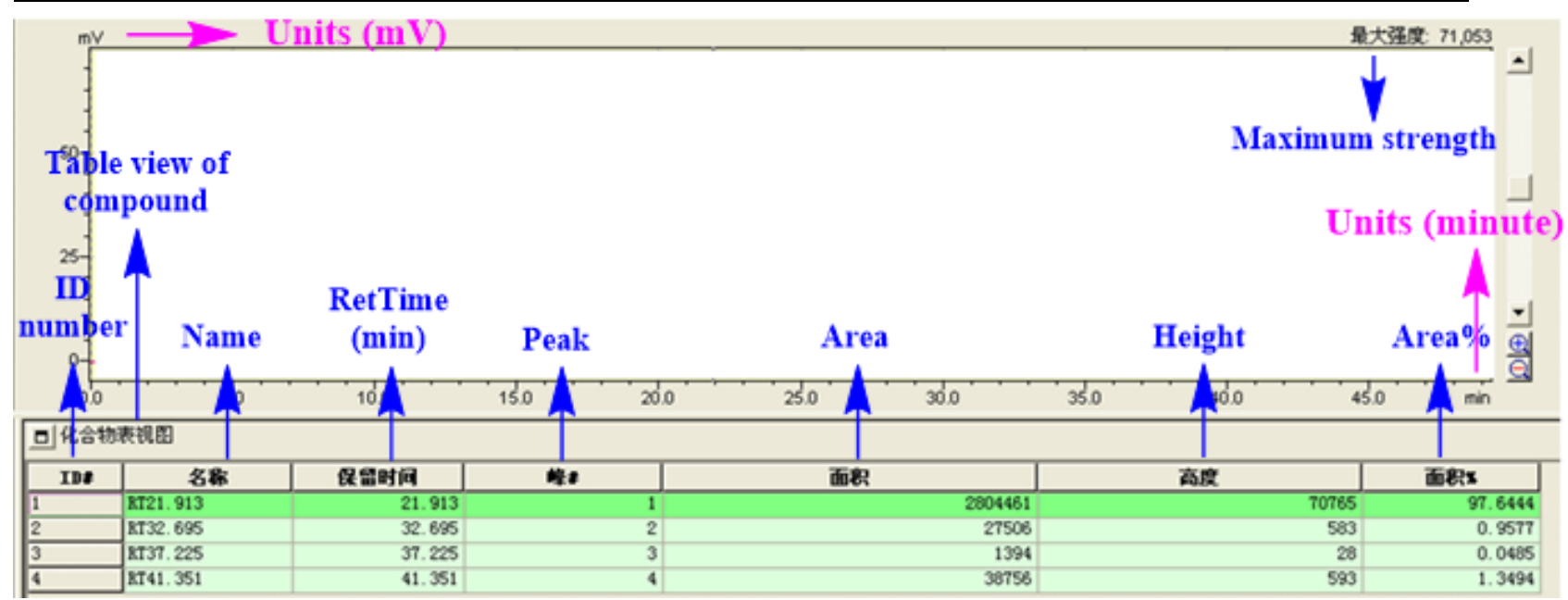


8g: (1S,4S)-1-(3-bromophenyl)-4-nitro-4-(phenylsulfonyl)pentan-1-ol: (HPLC: Chiracel OD-3, detected at $254 \mathrm{~nm}$, eluent: $\mathrm{n}$-hexane $/ 2$-propanol $=85 / 15$, flow rate $\left.=1.0 \mathrm{~mL} / \mathrm{min}, 25^{\circ} \mathrm{C}\right)$.
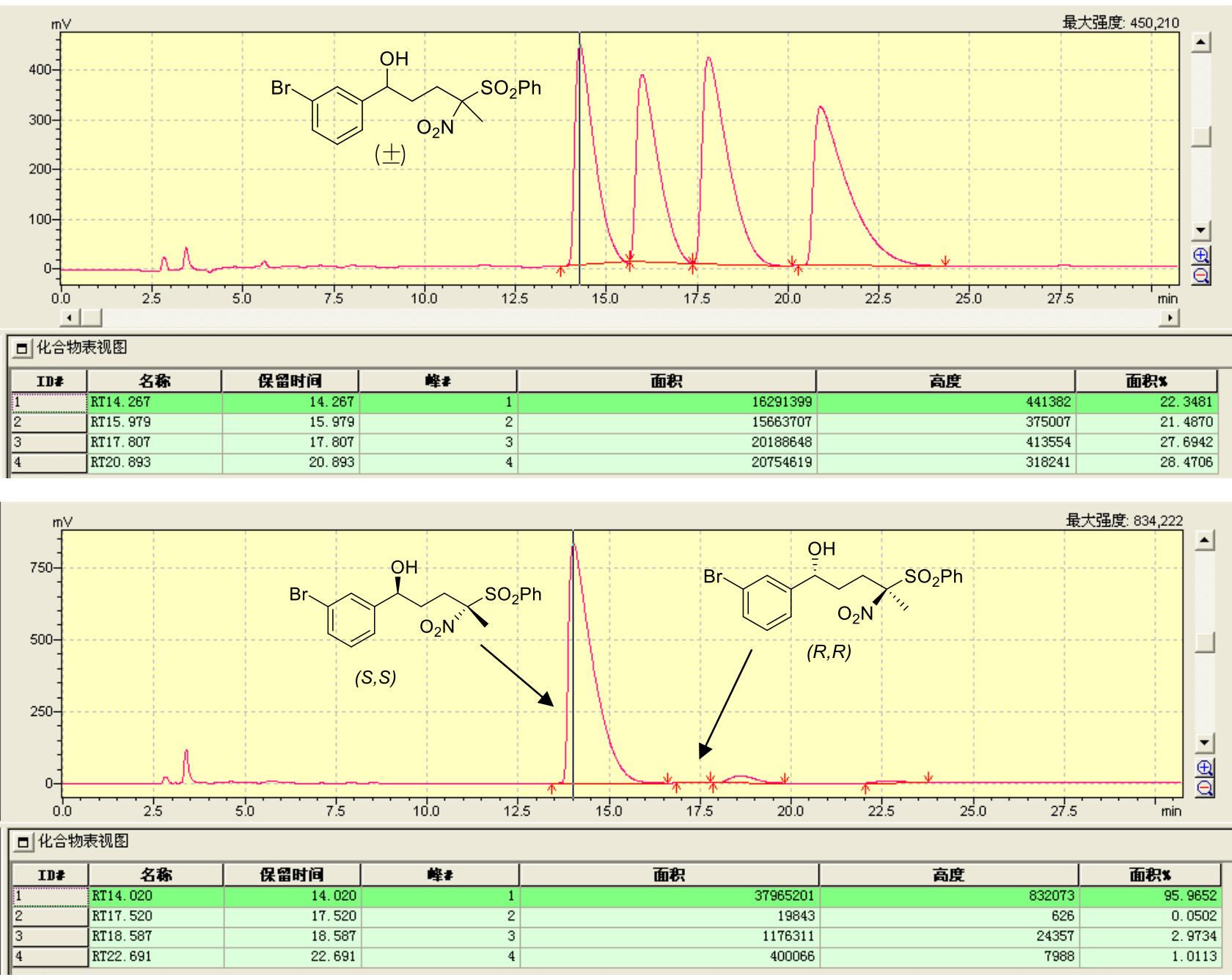

Translation of all characters (Chinese) in above two frameworks to English is as follows:

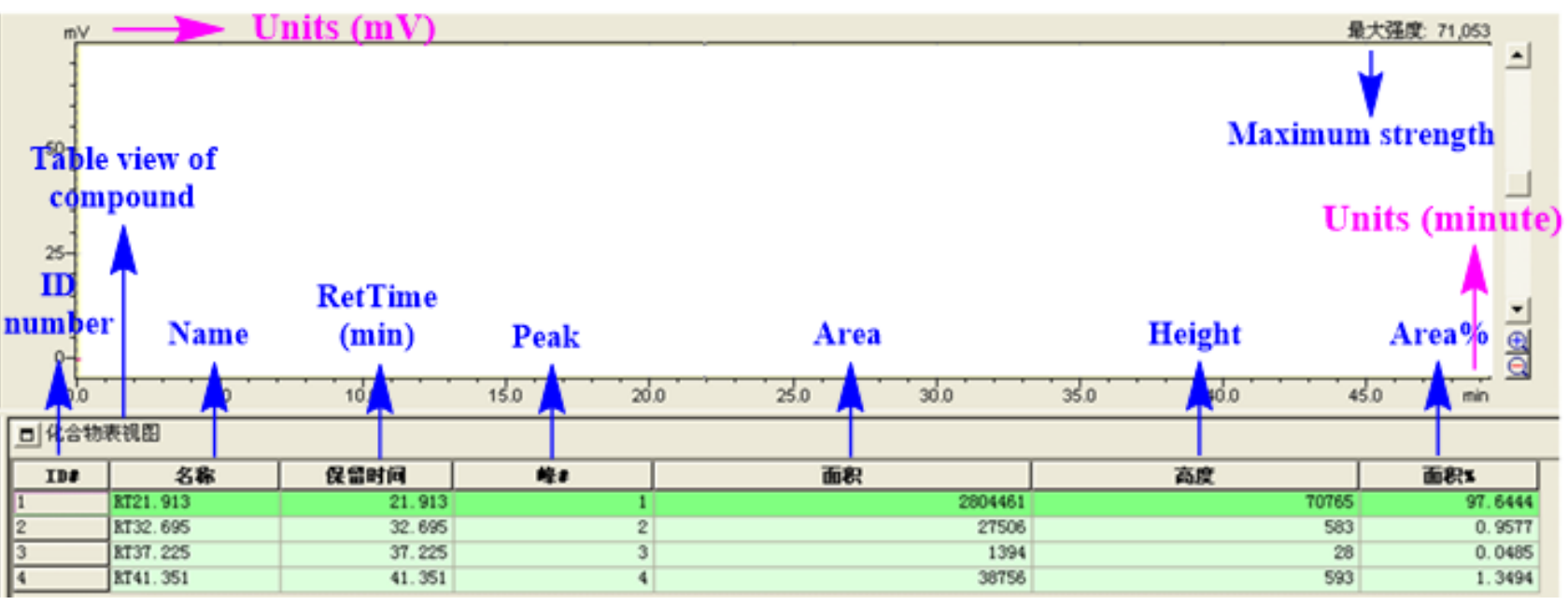


8h: (1S,4S)-1-(4-iodophenyl)-4-nitro-4-(phenylsulfonyl)pentan-1-ol: (HPLC: Chiracel OZ-H, detected at $254 \mathrm{~nm}$, eluent: $\mathrm{n}$-hexane $/ 2$-propanol $=90 / 10$, flow rate $=1.0 \mathrm{~mL} / \mathrm{min}, 25^{\circ} \mathrm{C}$ ).
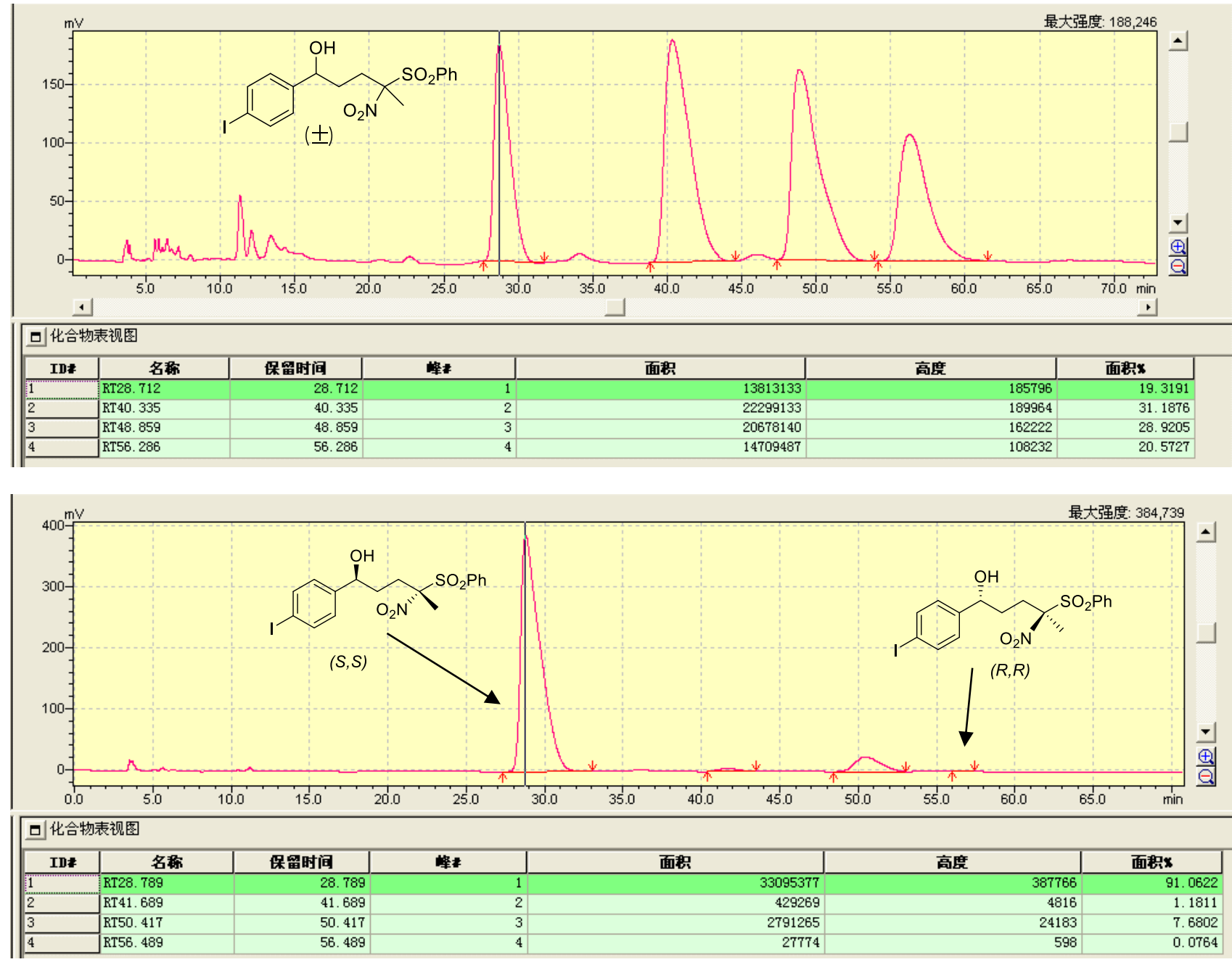

Translation of all characters (Chinese) in above two frameworks to English is as follows:

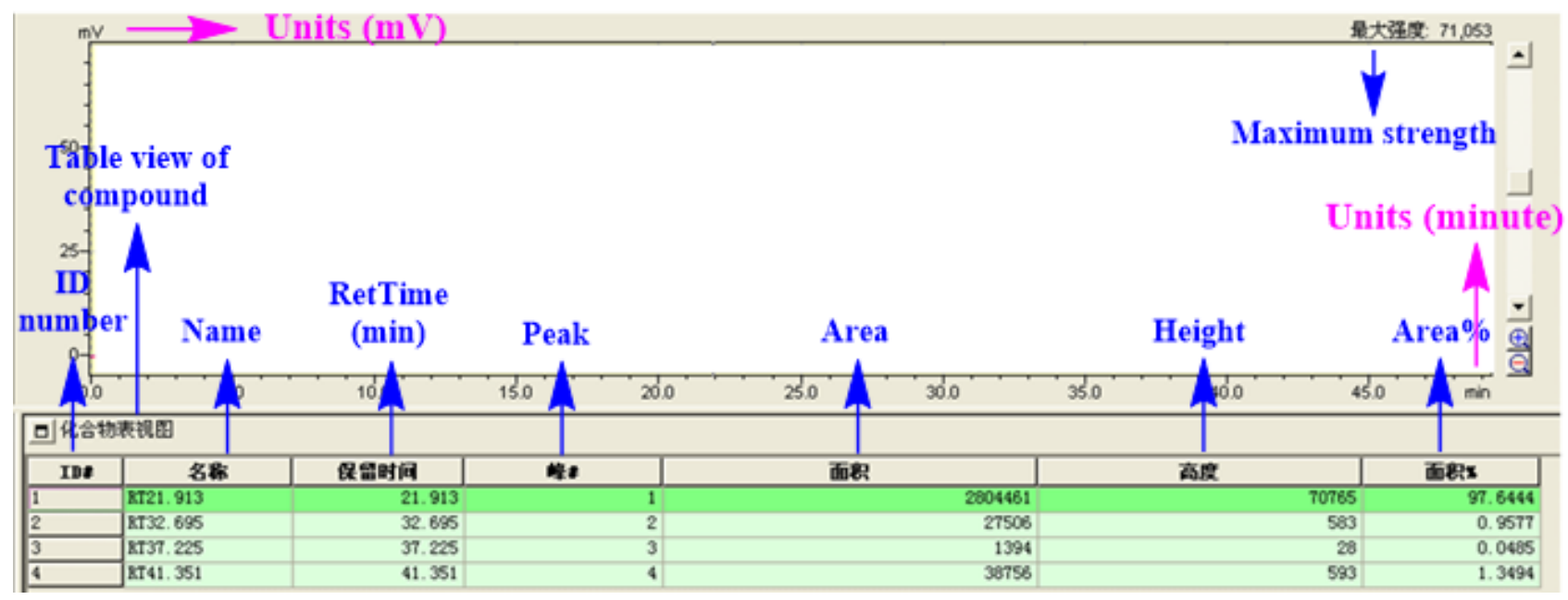


8i: (1S,4S)-4-nitro-1-(4-nitrophenyl)-4-(phenylsulfonyl)pentan-1-ol: (HPLC: Chiracel OZ-H, detected at $254 \mathrm{~nm}$, eluent: $\mathrm{n}$-hexane $/ 2$-propanol $=85 / 15$, flow rate $=1.0 \mathrm{~mL} / \mathrm{min}, 25^{\circ} \mathrm{C}$ ).
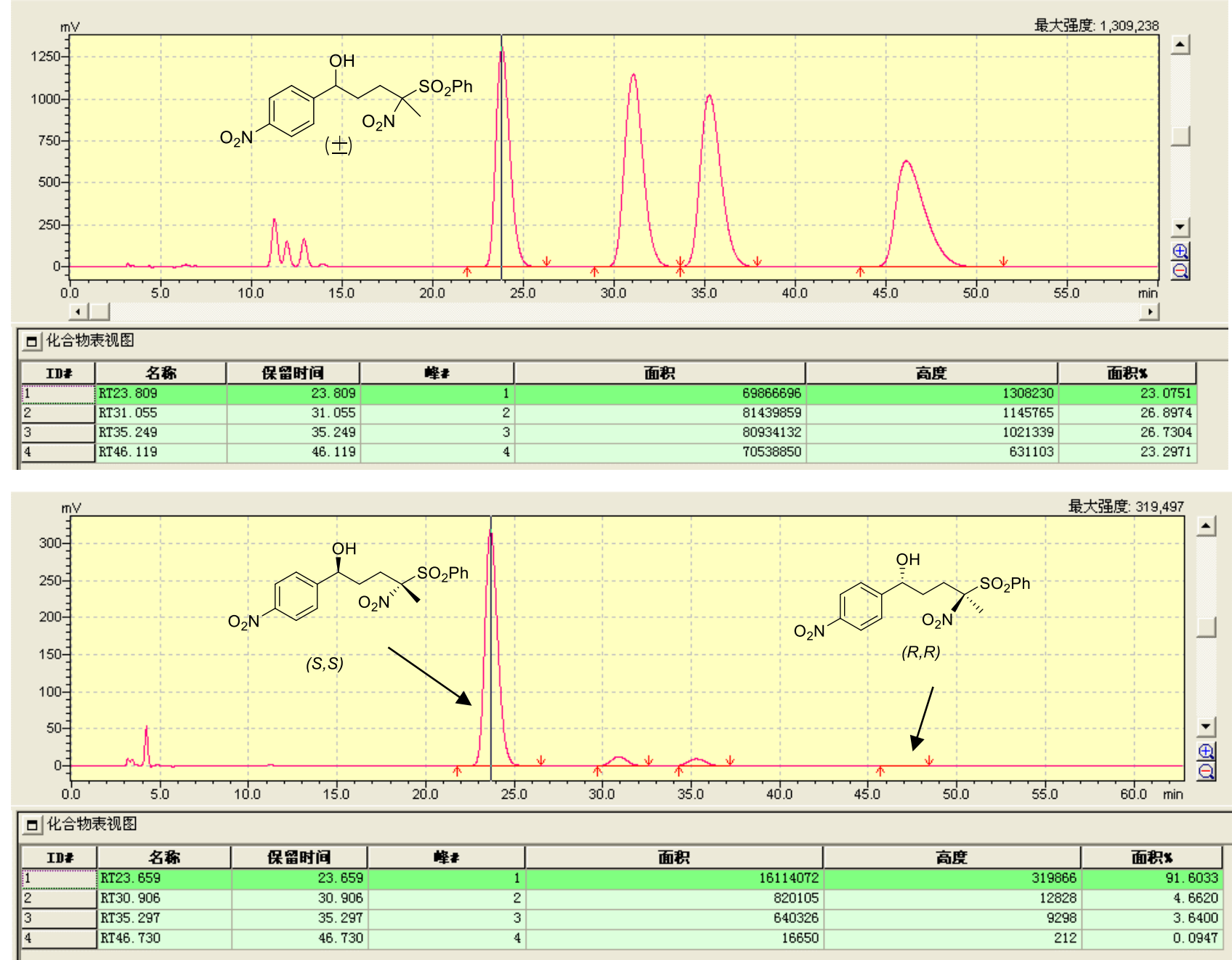

Translation of all characters (Chinese) in above two frameworks to English is as follows:

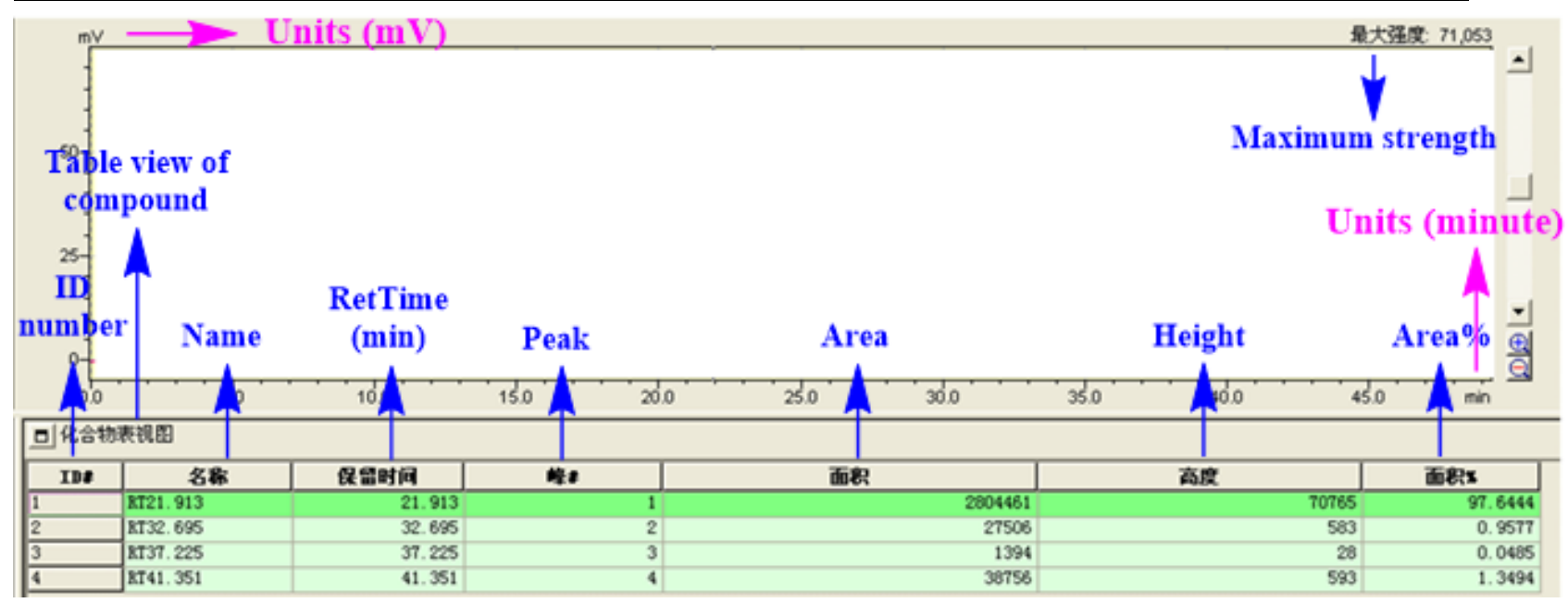


8j: 4-((1S,4S)-1-hydroxy-4-nitro-4-(phenylsulfonyl)pentyl)benzonitrile: (HPLC: Chiracel OZ-H, detected at $254 \mathrm{~nm}$, eluent: $\mathrm{n}$-hexane $/ 2$-propanol $=90 / 10$, flow rate $\left.=1.0 \mathrm{~mL} / \mathrm{min}, 25^{\circ} \mathrm{C}\right)$.
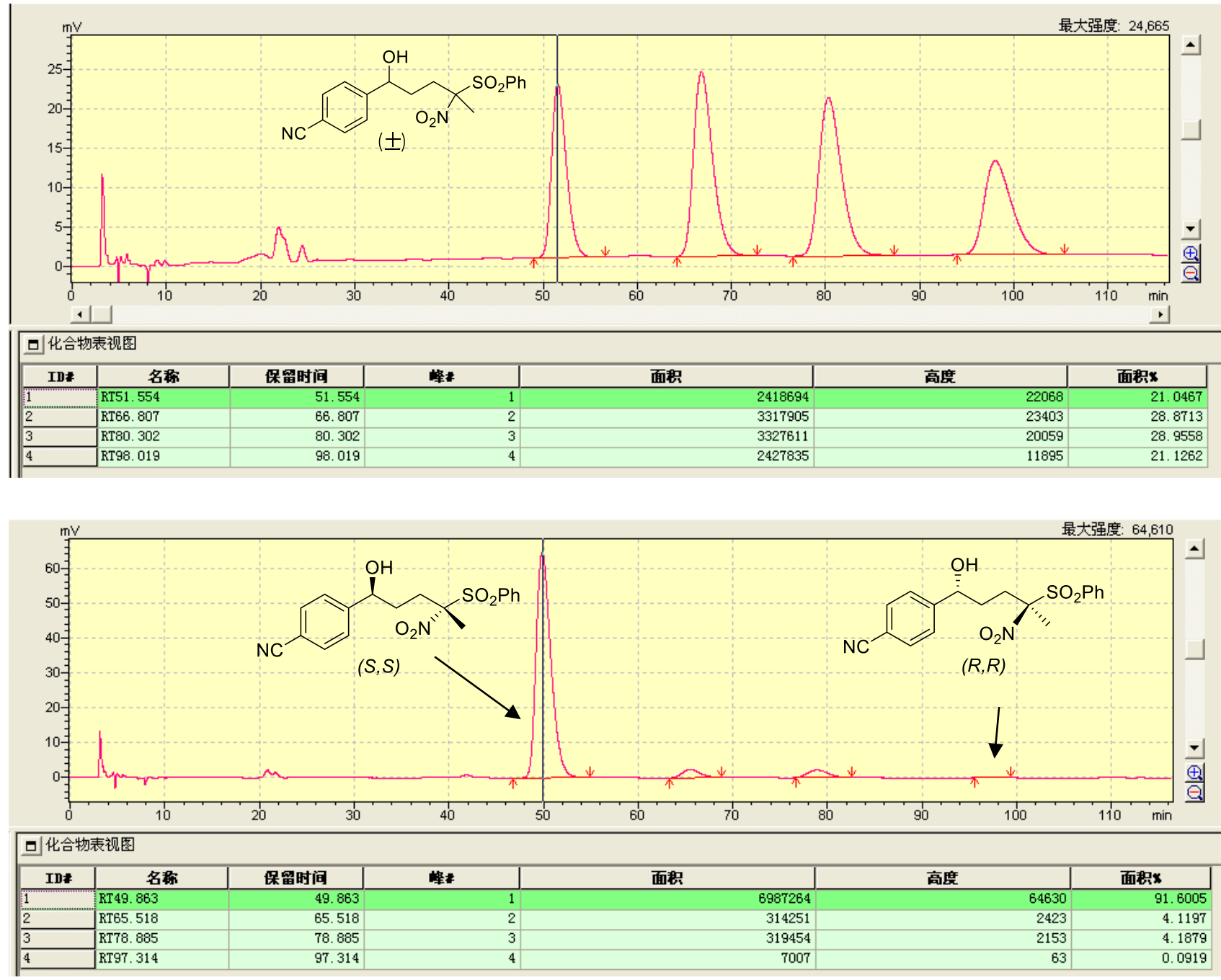

Translation of all characters (Chinese) in above two frameworks to English is as follows:

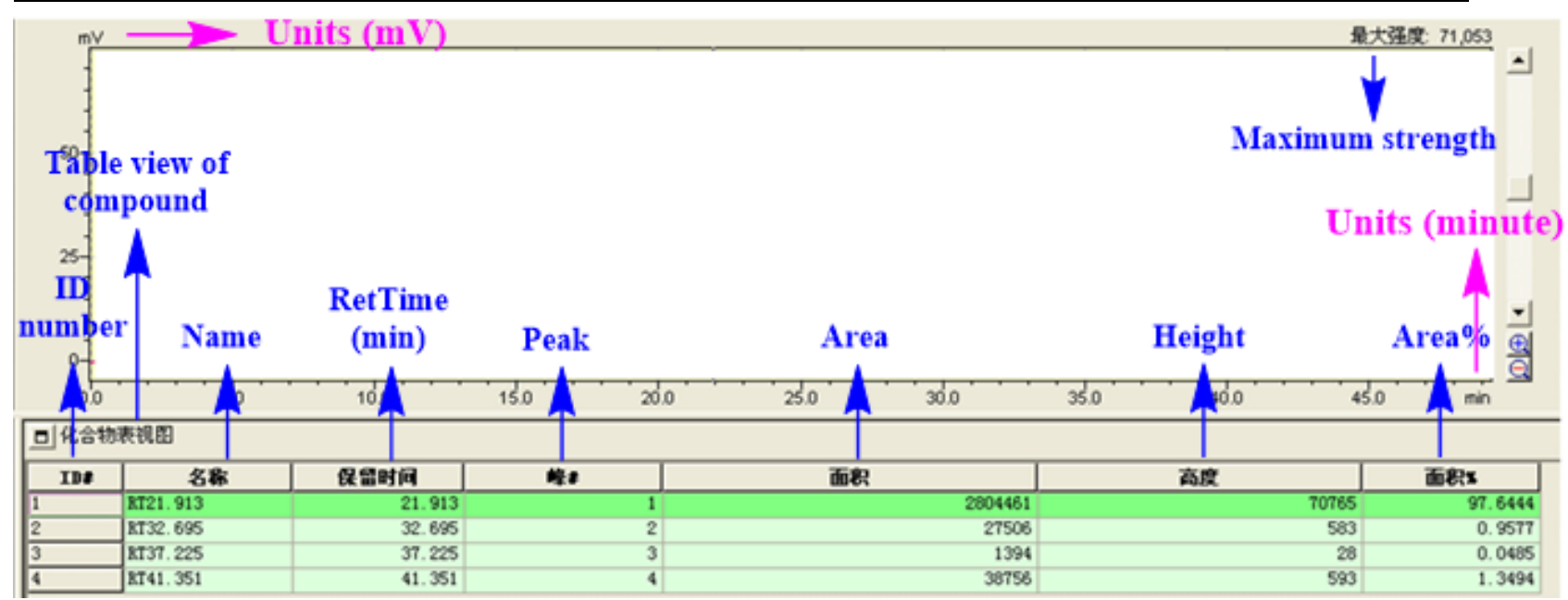


8k: (1S,4S)-1-(3-methoxyphenyl)-4-nitro-4-(phenylsulfonyl)pentan-1-ol: (HPLC: Chiracel OZ-H, detected at $254 \mathrm{~nm}$, eluent: $\mathrm{n}$-hexane $/ 2$-propanol $=85 / 15$, flow rate $=1.0 \mathrm{~mL} / \mathrm{min}, 25^{\circ} \mathrm{C}$ ).
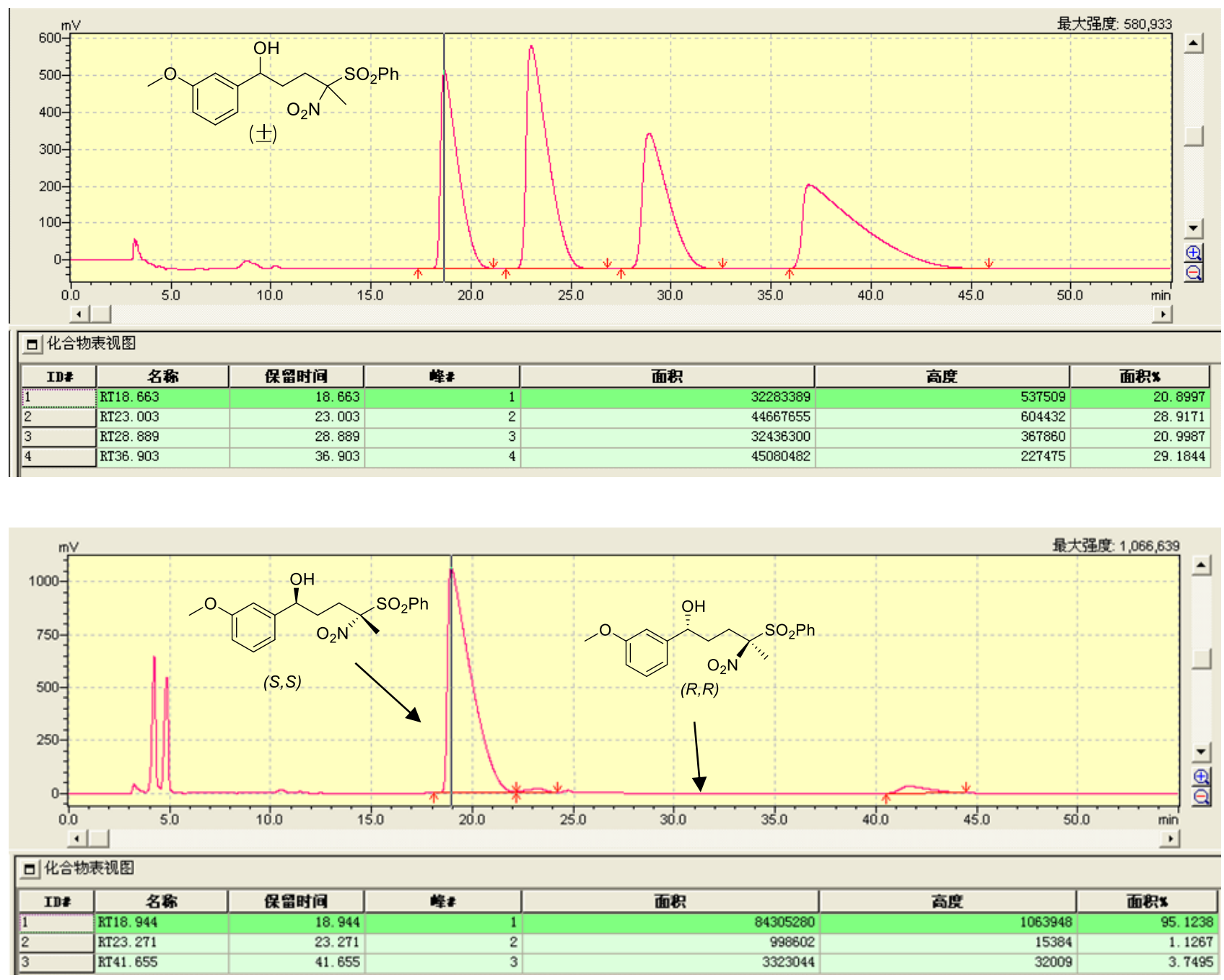

Translation of all characters (Chinese) in above two frameworks to English is as follows:

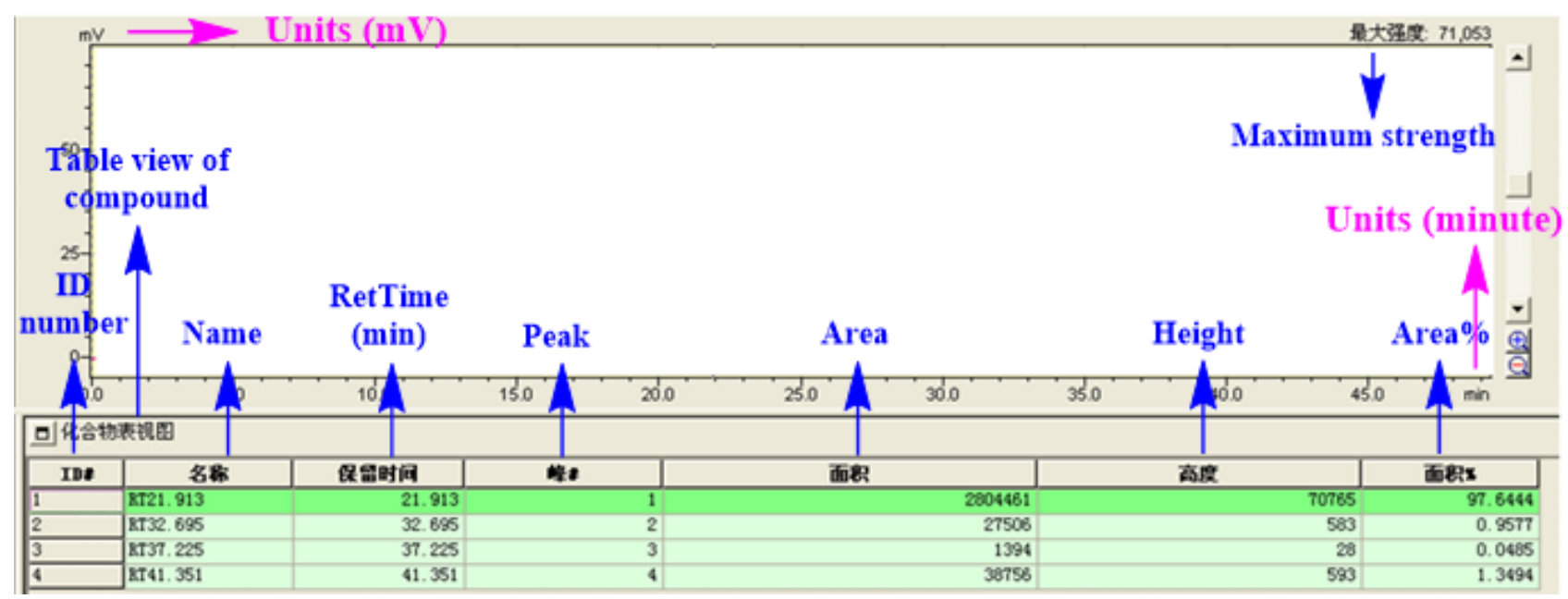




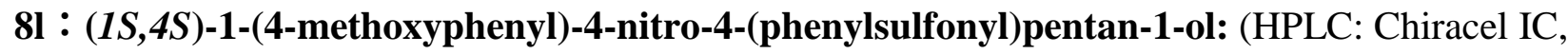
detected at $254 \mathrm{~nm}$, eluent: $\mathrm{n}$-hexane $/ 2$-propanol $=85 / 15$, flow rate $=1.0 \mathrm{~mL} / \mathrm{min}, 25^{\circ} \mathrm{C}$ ).
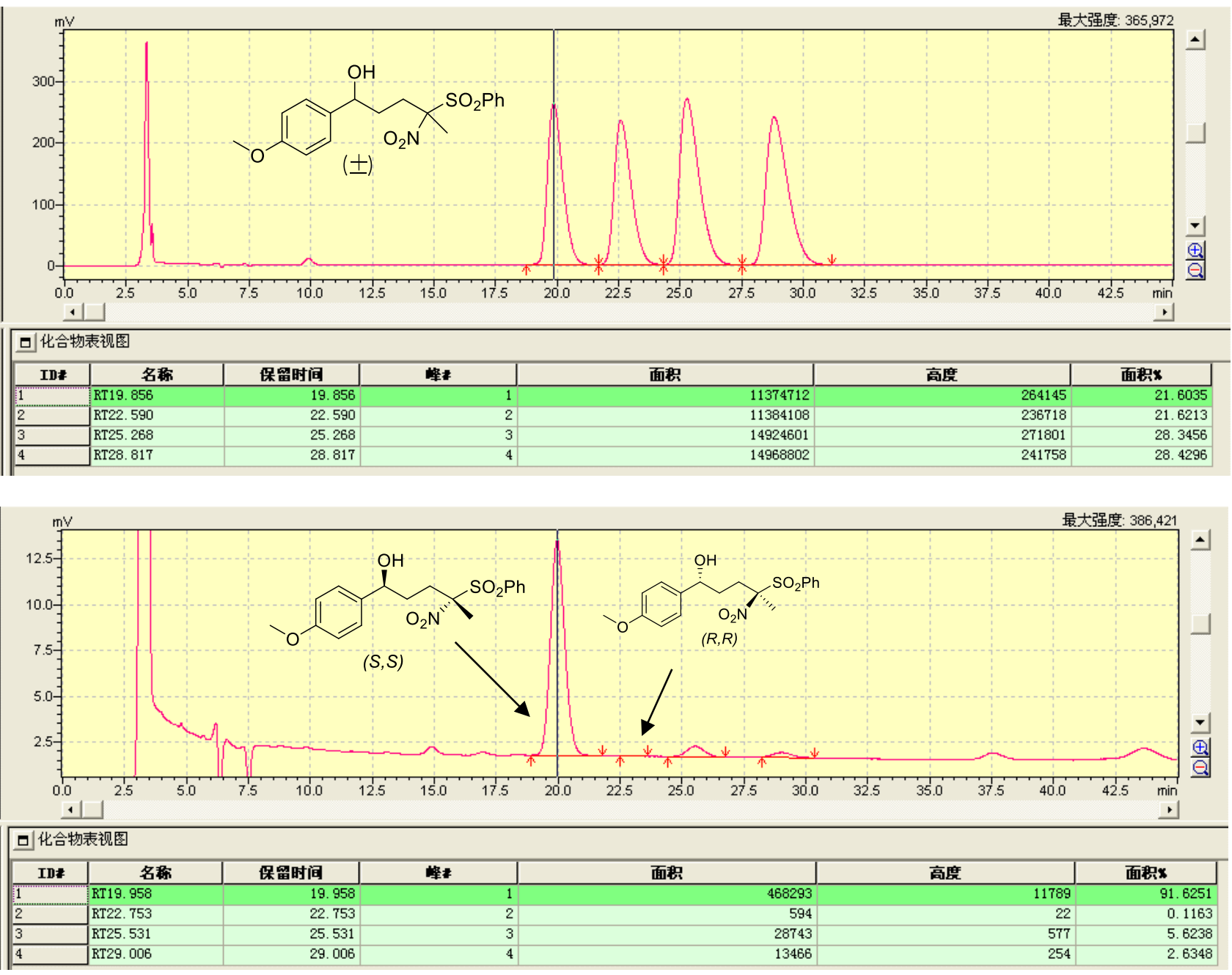

Translation of all characters (Chinese) in above two frameworks to English is as follows:

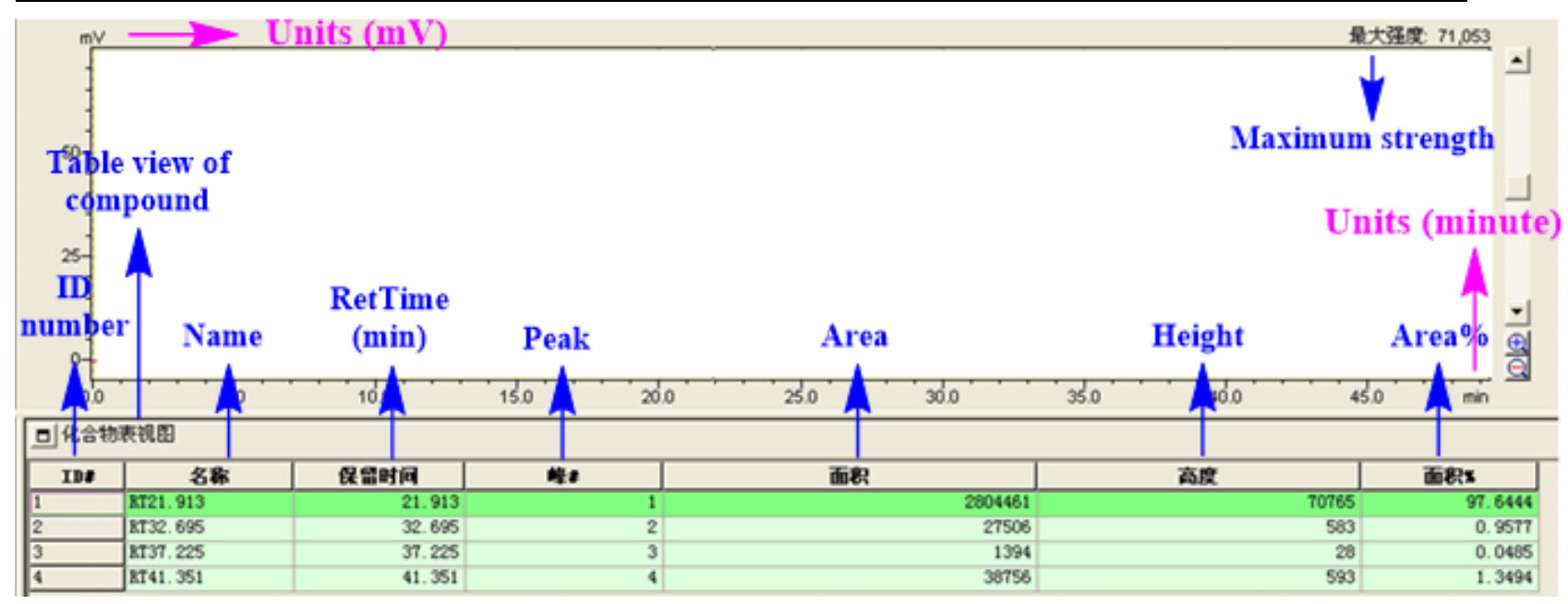


8m: (1S,4S)-4-nitro-4-(phenylsulfonyl)-1-(p-tolyl)pentan-1-ol: (HPLC: Chiracel OD-3, detected at $254 \mathrm{~nm}$, eluent: $\mathrm{n}$-hexane $/ 2$-propanol $=85 / 15$, flow rate $=1.0 \mathrm{~mL} / \mathrm{min}, 25^{\circ} \mathrm{C}$ ).
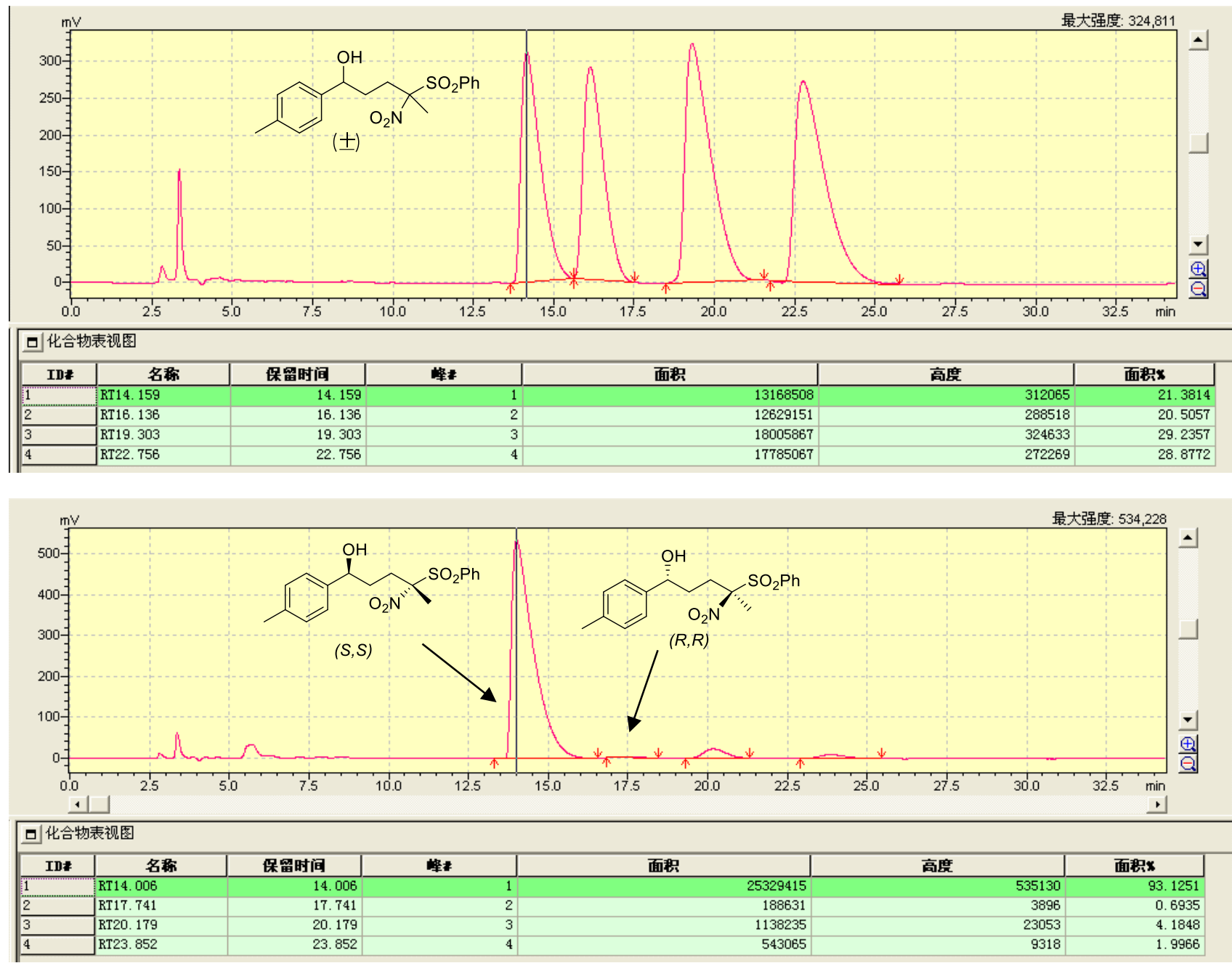

Translation of all characters (Chinese) in above two frameworks to English is as follows:

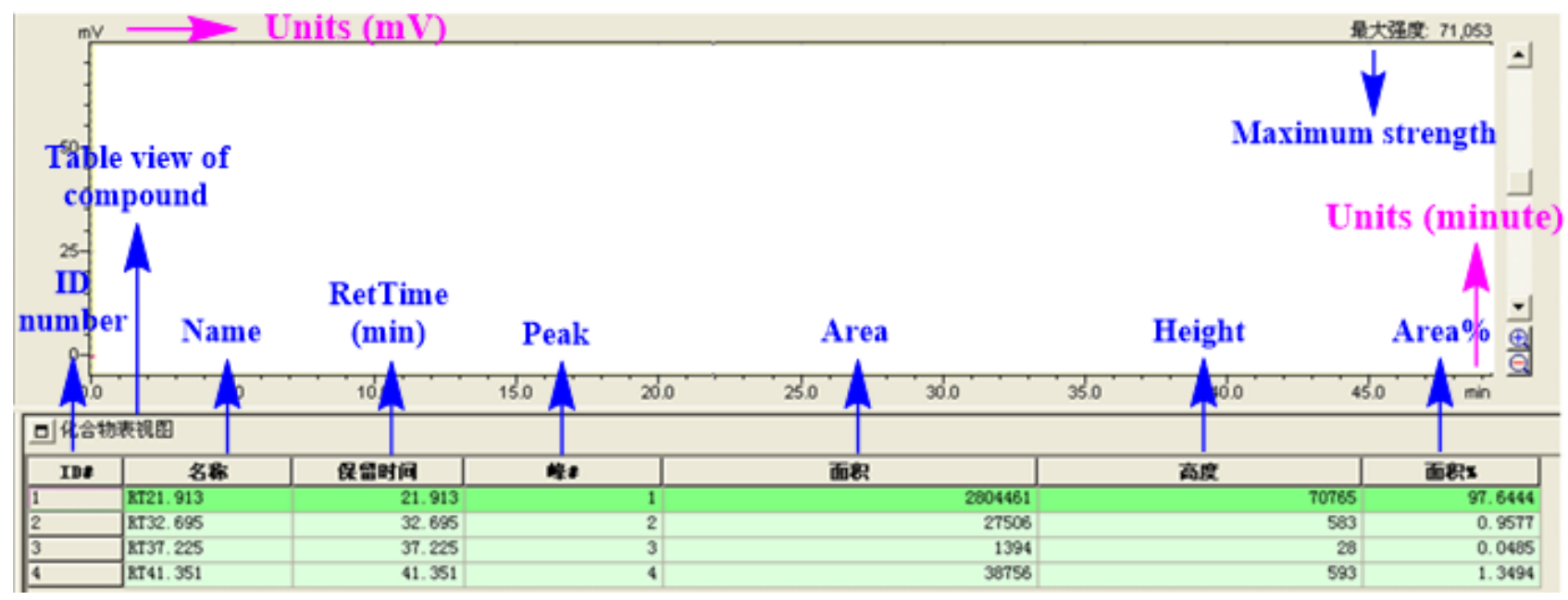


8n: (1S,4S)-4-nitro-4-(phenylsulfonyl)-1-(m-tolyl)pentan-1-ol: (HPLC: Chiracel OZ-H, detected at $254 \mathrm{~nm}$, eluent: $\mathrm{n}$-hexane $/ 2$-propanol $=75 / 25$, flow rate $=1.0 \mathrm{~mL} / \mathrm{min}, 25^{\circ} \mathrm{C}$ ).
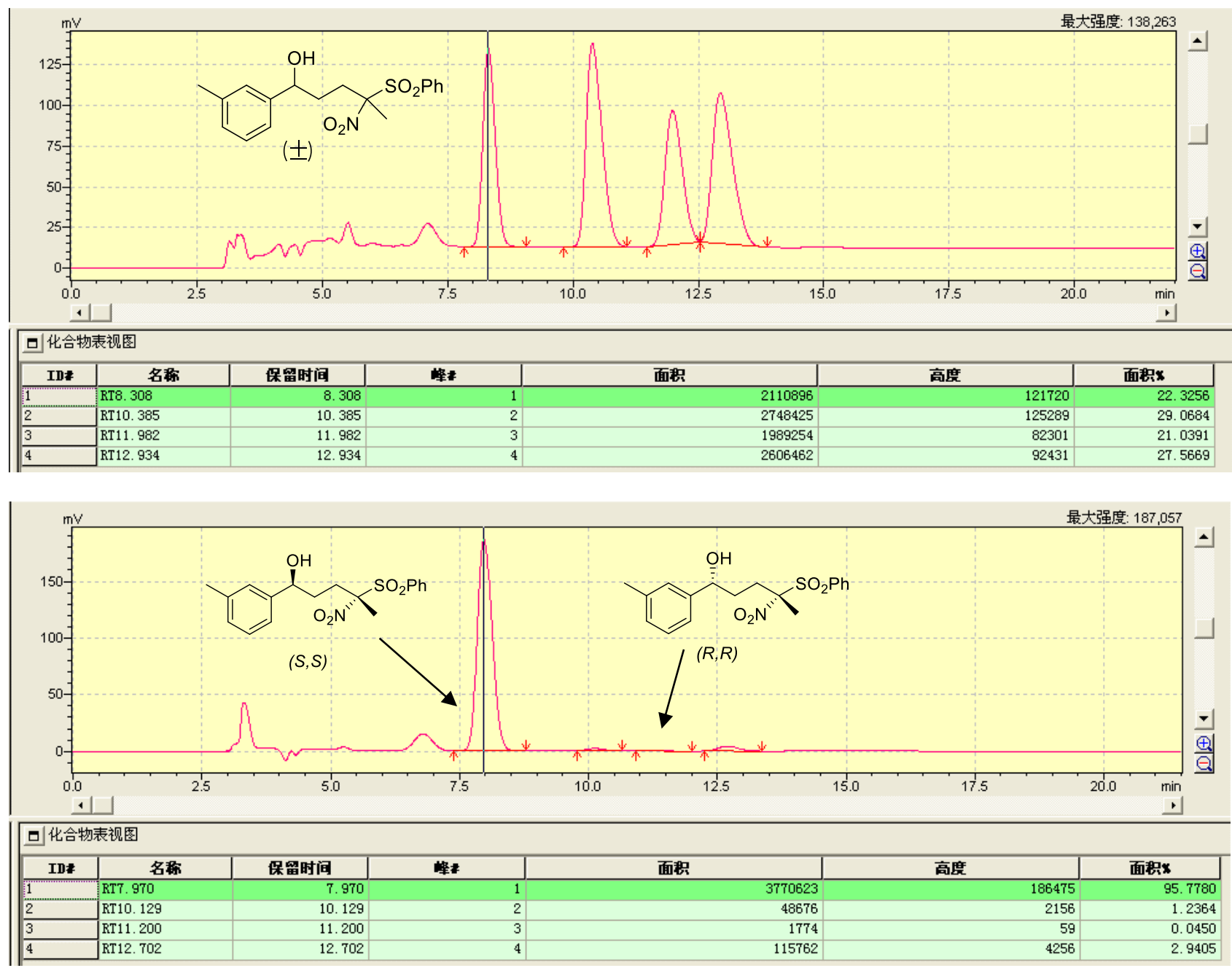

Translation of all characters (Chinese) in above two frameworks to English is as follows:

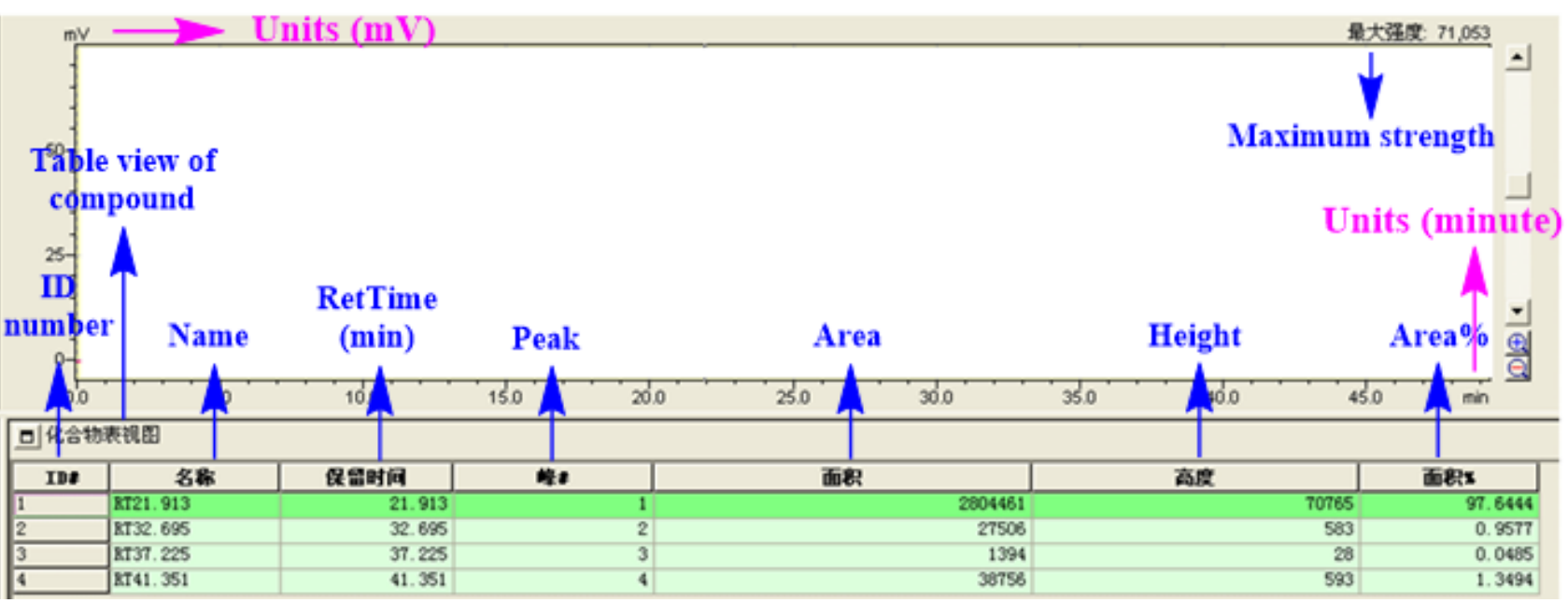


8o: (1S,4S)-1-(naphthalen-2-yl)-4-nitro-4-(phenylsulfonyl)pentan-1-ol: (HPLC: Chiracel IC, detected at $254 \mathrm{~nm}$, eluent: $\mathrm{n}$-hexane $/ 2$-propanol $=90 / 10$, flow rate $=1.0 \mathrm{~mL} / \mathrm{min}, 25^{\circ} \mathrm{C}$ ).
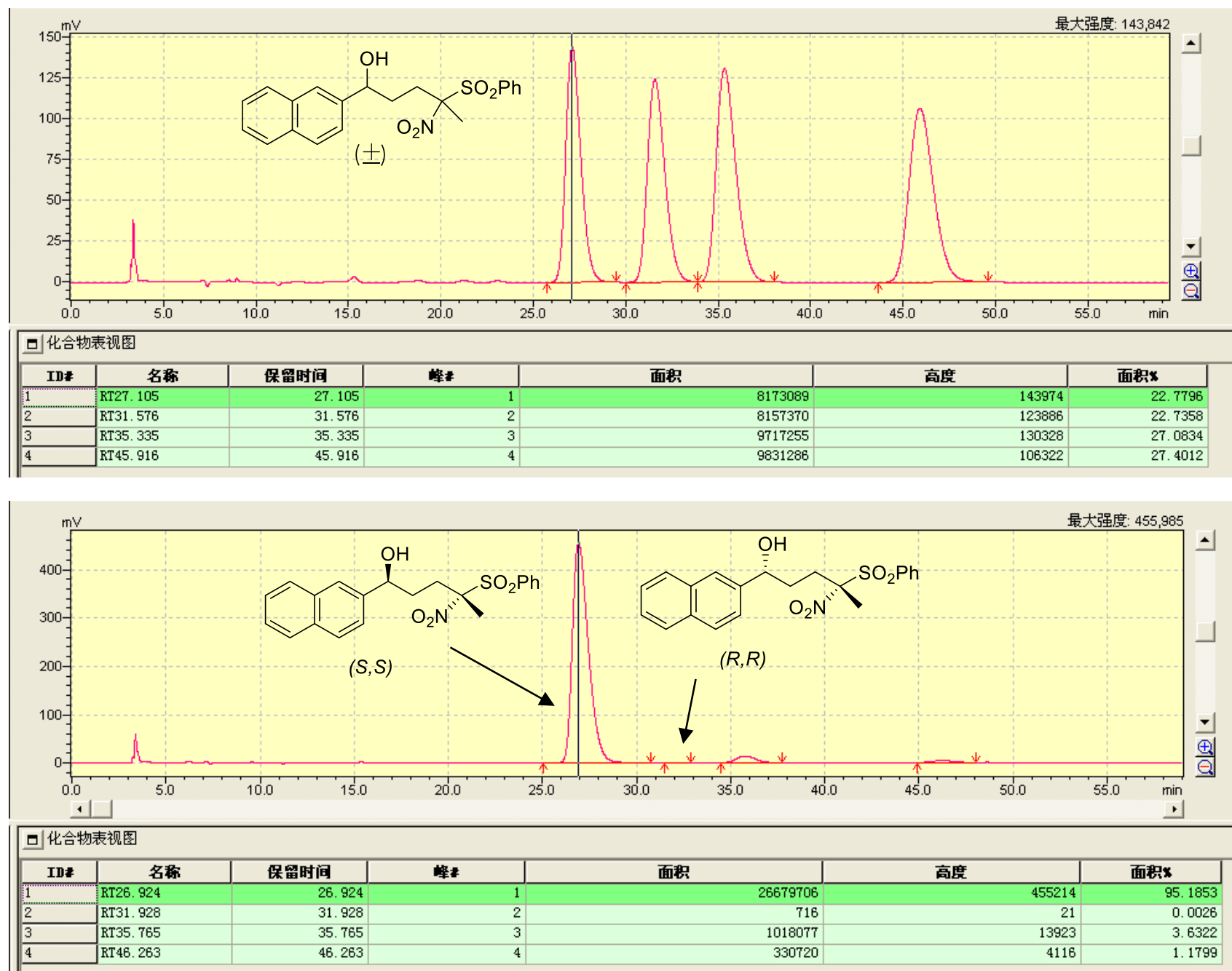

Translation of all characters (Chinese) in above two frameworks to English is as follows:

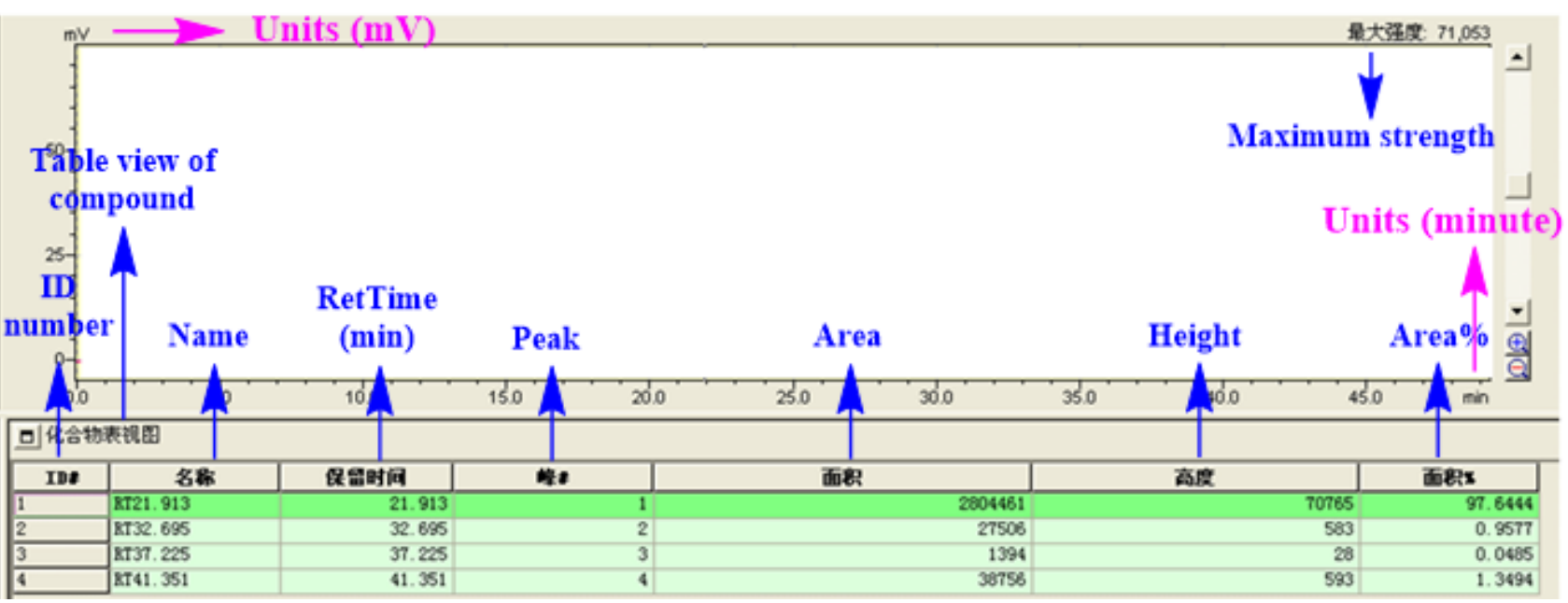


8p: (1S,4S)-1-(furan-2-yl)-4-nitro-4-(phenylsulfonyl)pentan-1-ol: (HPLC: Chiracel IC, detected at $254 \mathrm{~nm}$, eluent: $\mathrm{n}$-hexane $/ 2$-propanol $=95 / 5$, flow rate $=1.0 \mathrm{~mL} / \mathrm{min}, 25^{\circ} \mathrm{C}$ ).
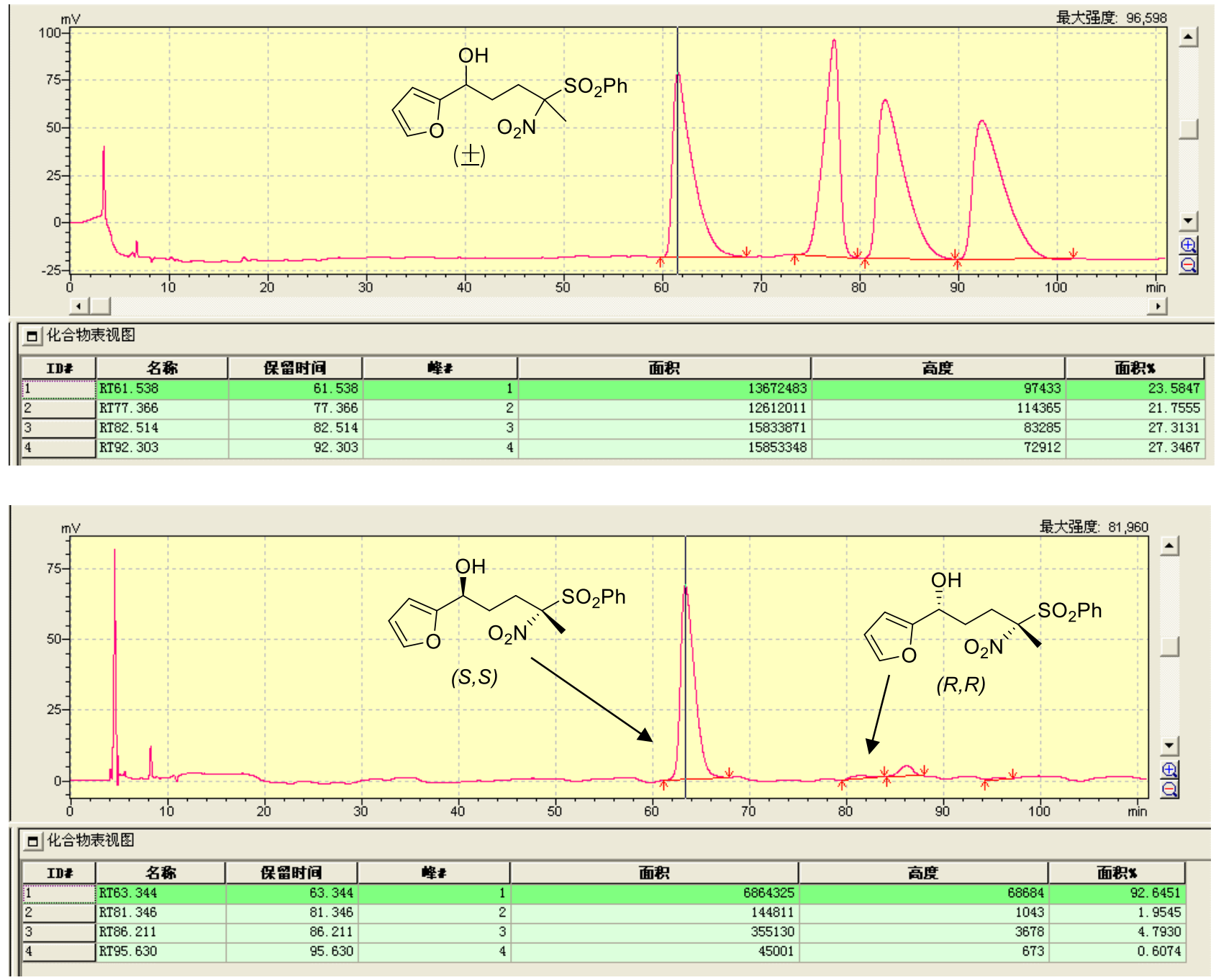

Translation of all characters (Chinese) in above two frameworks to English is as follows:

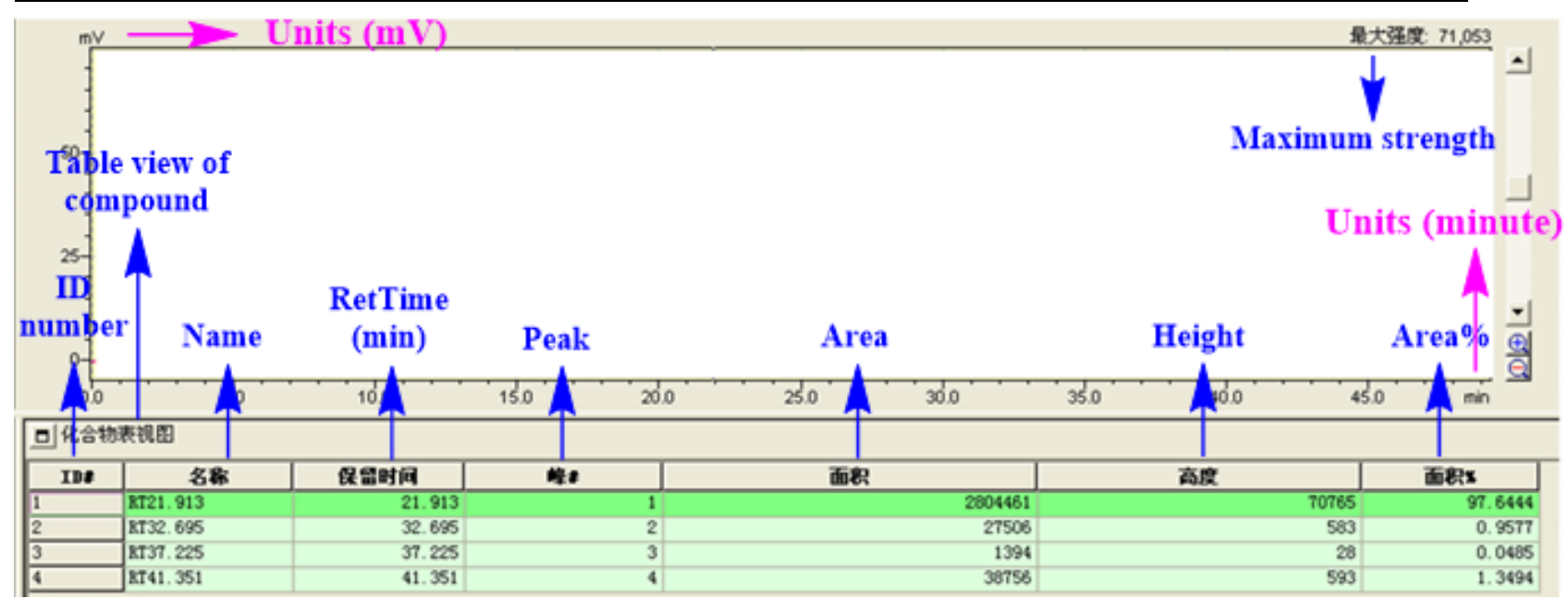


8q: (1S,4S)-4-nitro-4-(phenylsulfonyl)-1-(thiophen-2-yl)pentan-1-ol: (HPLC: Chiracel OD-3, detected at $254 \mathrm{~nm}$, eluent: $\mathrm{n}$-hexane $/ 2$-propanol $=85 / 15$, flow rate $=1.0 \mathrm{~mL} / \mathrm{min}, 25^{\circ} \mathrm{C}$ ).

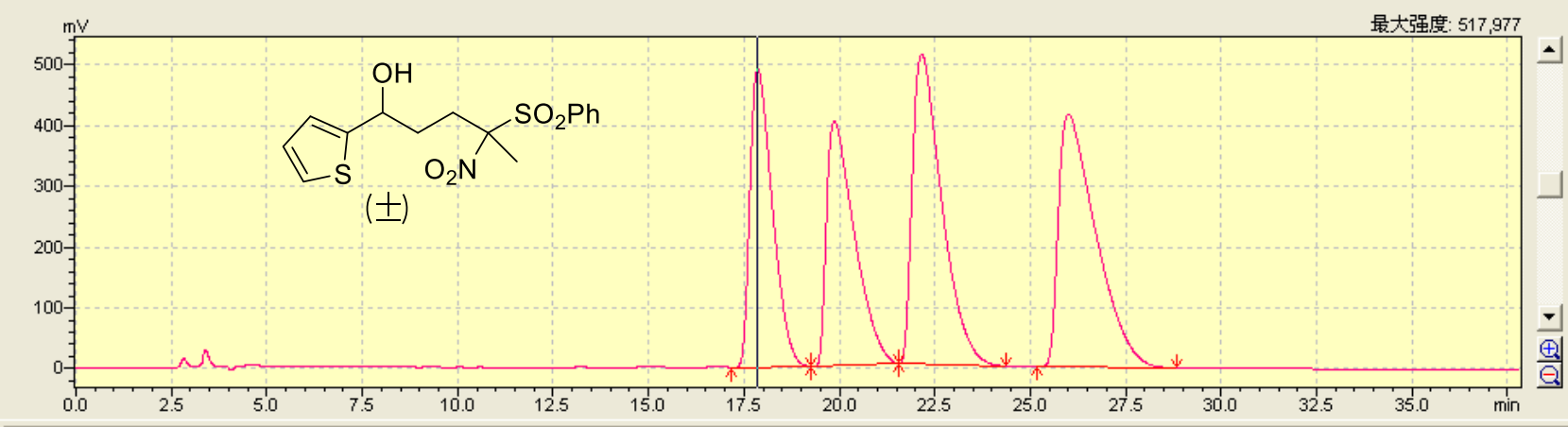

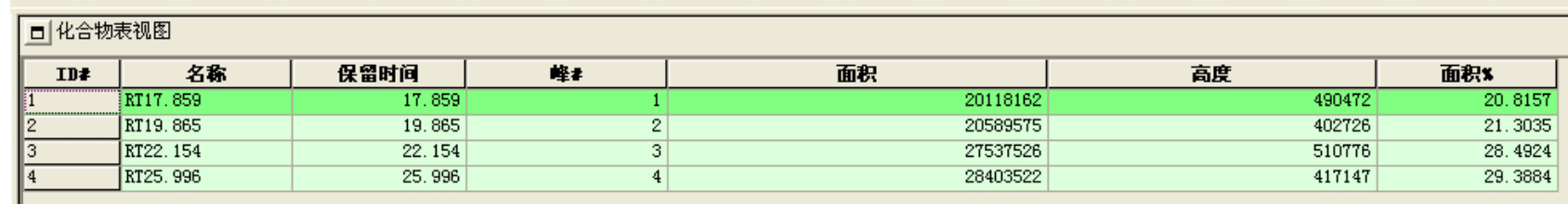

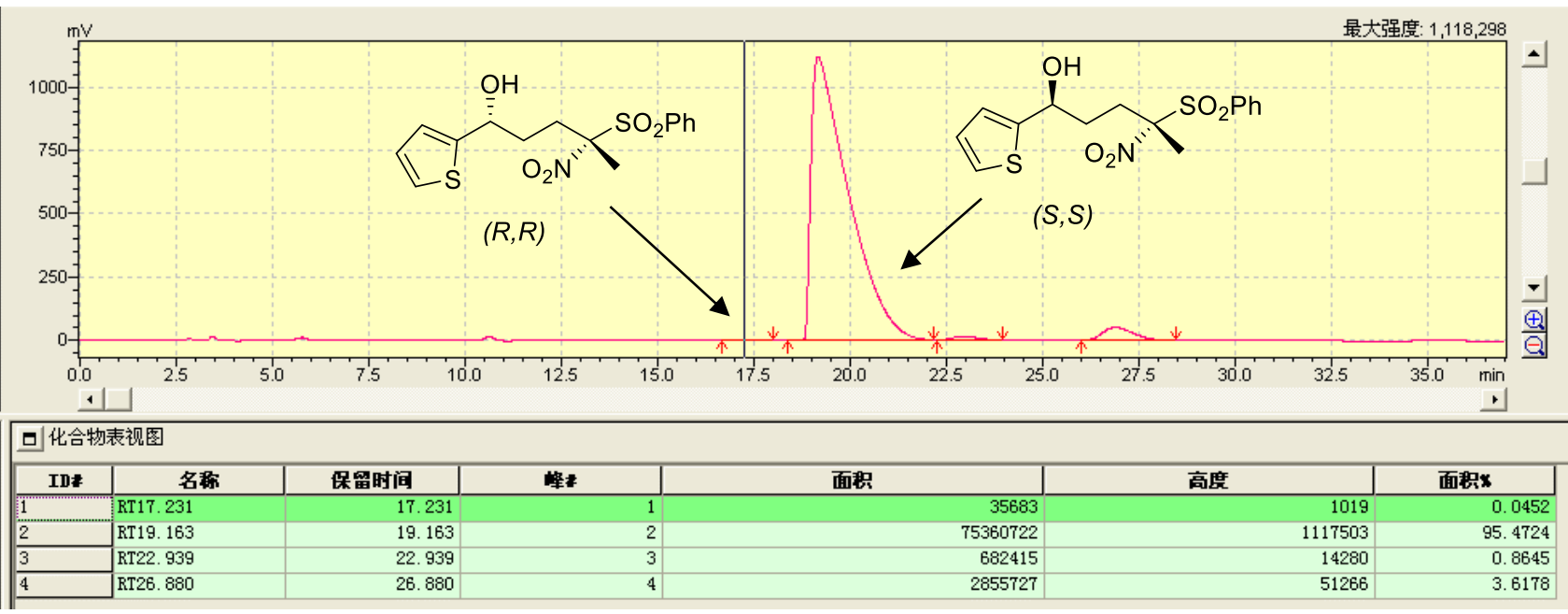

Translation of all characters (Chinese) in above two frameworks to English is as follows:

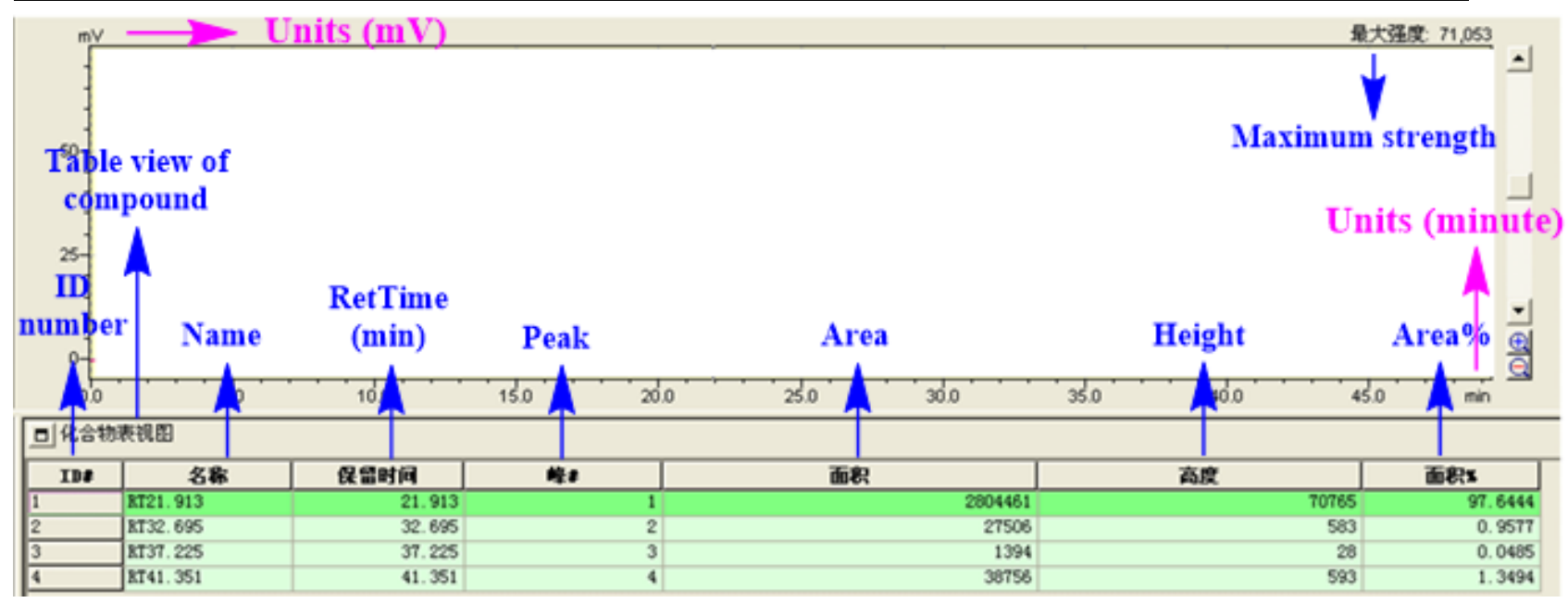


8r: (1S,4S)-1-(3,9-dihydropyren-4-yl)-4-nitro-4-(phenylsulfonyl)pentan-1-ol: (HPLC: Chiracel IC, detected at $254 \mathrm{~nm}$, eluent: $\mathrm{n}$-hexane $/ 2$-propanol $=90 / 10$, flow rate $=0.7 \mathrm{~mL} / \mathrm{min}, 25^{\circ} \mathrm{C}$ ).
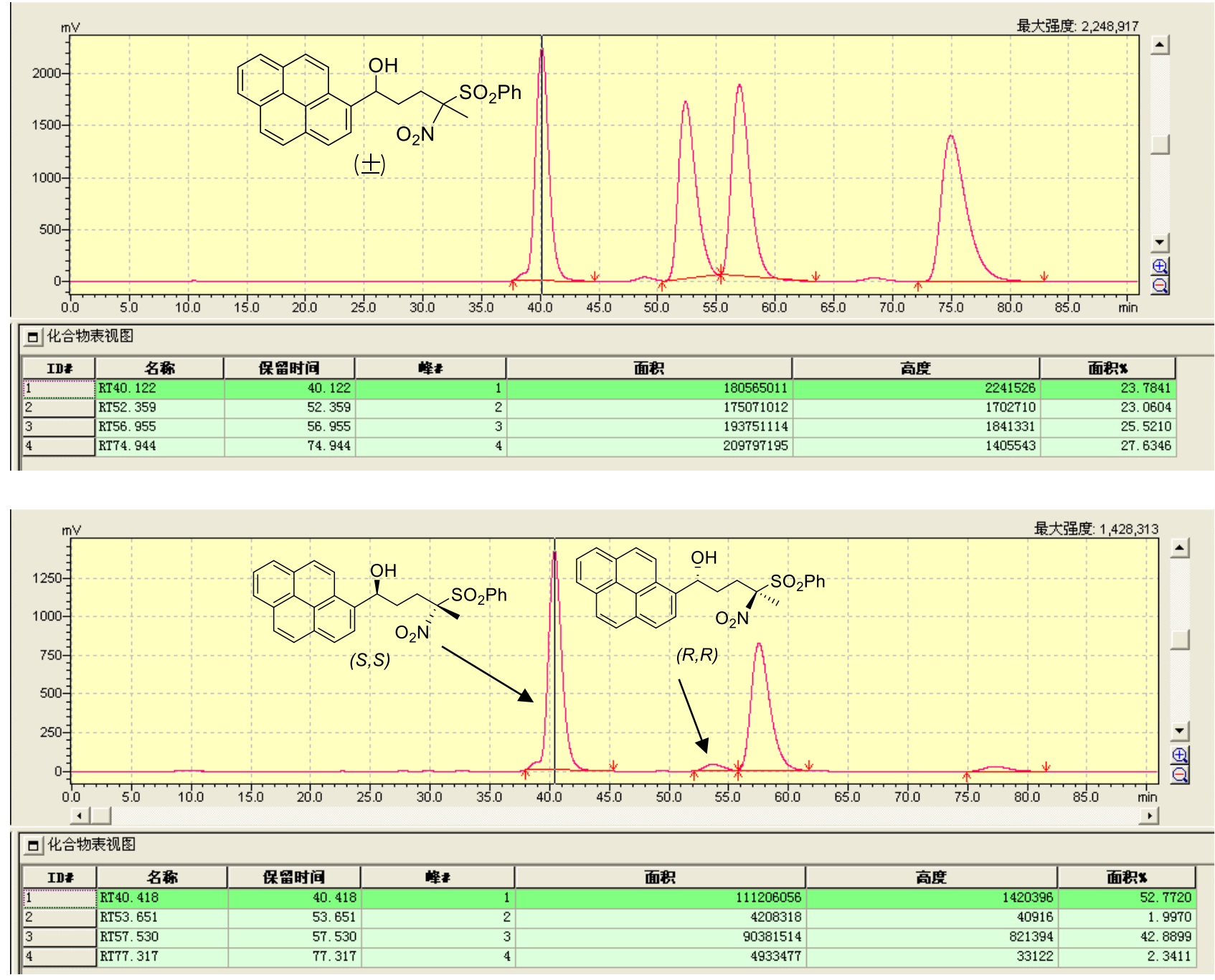

Translation of all characters (Chinese) in above two frameworks to English is as follows:

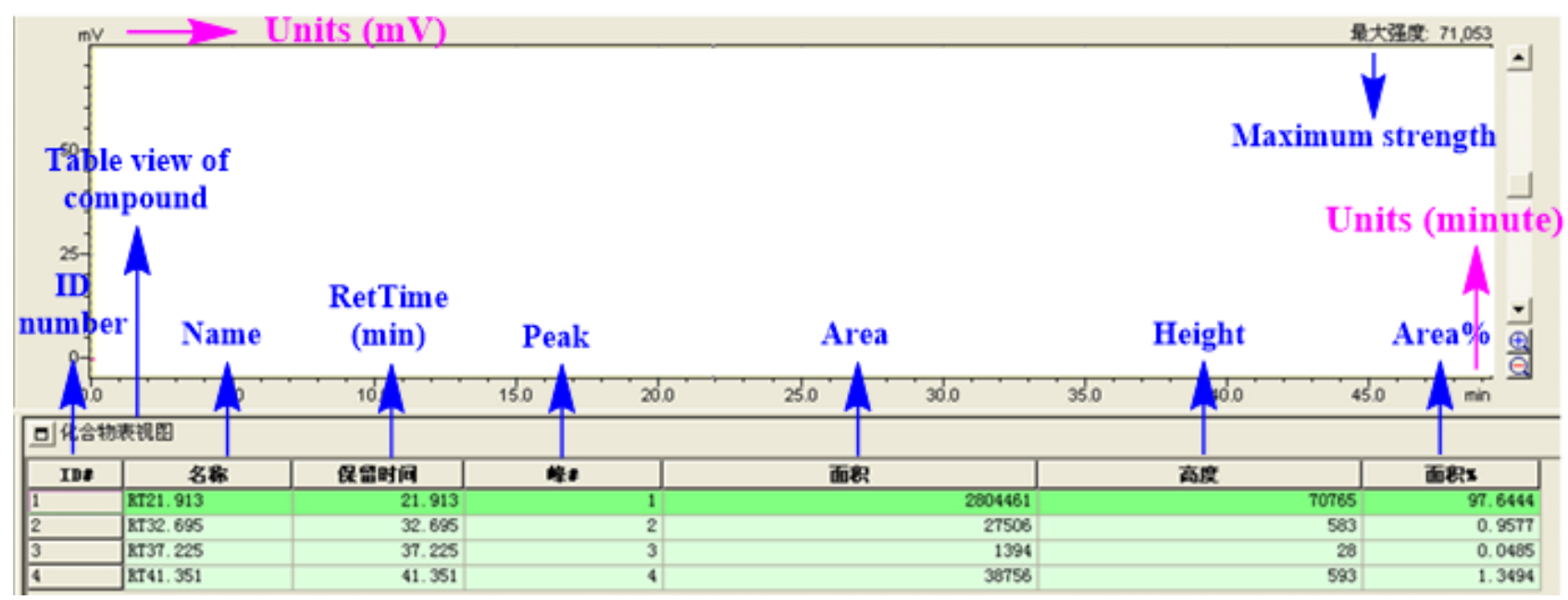

Figure S9. HPLC analyses for chiral products. 
Table S3. Reusability of the co-catalyst system. ${ }^{\mathrm{a}}$

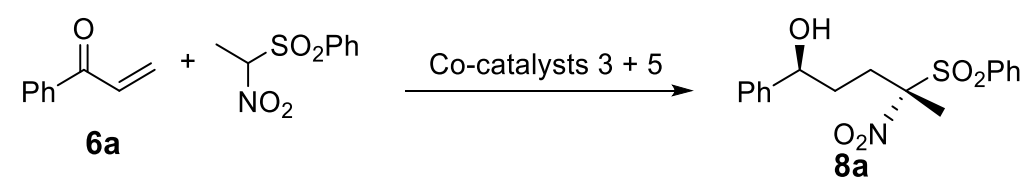

\begin{tabular}{lllllll}
\hline Entry & 1 & 2 & 3 & 4 & 5 & 6 \\
\hline \%Yield & 93 & 93 & 91 & 91 & 90 & 89 \\
\%ee & 99 & 99 & 99 & 99 & 99 & 99 \\
$d r$ & $98: 2$ & $98: 2$ & $98: 2$ & $98: 2$ & $97: 3$ & $94: 6$ \\
\hline
\end{tabular}

${ }^{a}$ Reaction conditions: Co-catalysts ( $92.60 \mathrm{mg}$ of $\mathbf{3}$ and $137.80 \mathrm{mg}$ of 5), 1-phenylprop-2-en-1-one (1.0 mmol), ((1-nitroethyl)sulfonyl)benzene (1.20 mmol), $\mathrm{HCO}_{2} \mathrm{Na}(10.0 \mathrm{mmol})$, and $20.0 \mathrm{~mL}$ of toluene $/ \mathrm{H}_{2} \mathrm{O}(\mathrm{v} / \mathrm{v}=1: 1)$ were added sequentially to a $100.0 \mathrm{~mL}$ round-bottom flask. The mixture was then stirred at $-35{ }^{\circ} \mathrm{C}$ for the first $8 \mathrm{~h}$ followed by at $35^{\circ} \mathrm{C}$ for the second $10 \mathrm{~h}$. Yields were determined by ${ }^{1} \mathrm{H}-\mathrm{NMR}$ analysis, and $e e$ and $d r$ values were determined by chiral HPLC analysis.

\section{Recycling experiment part:}

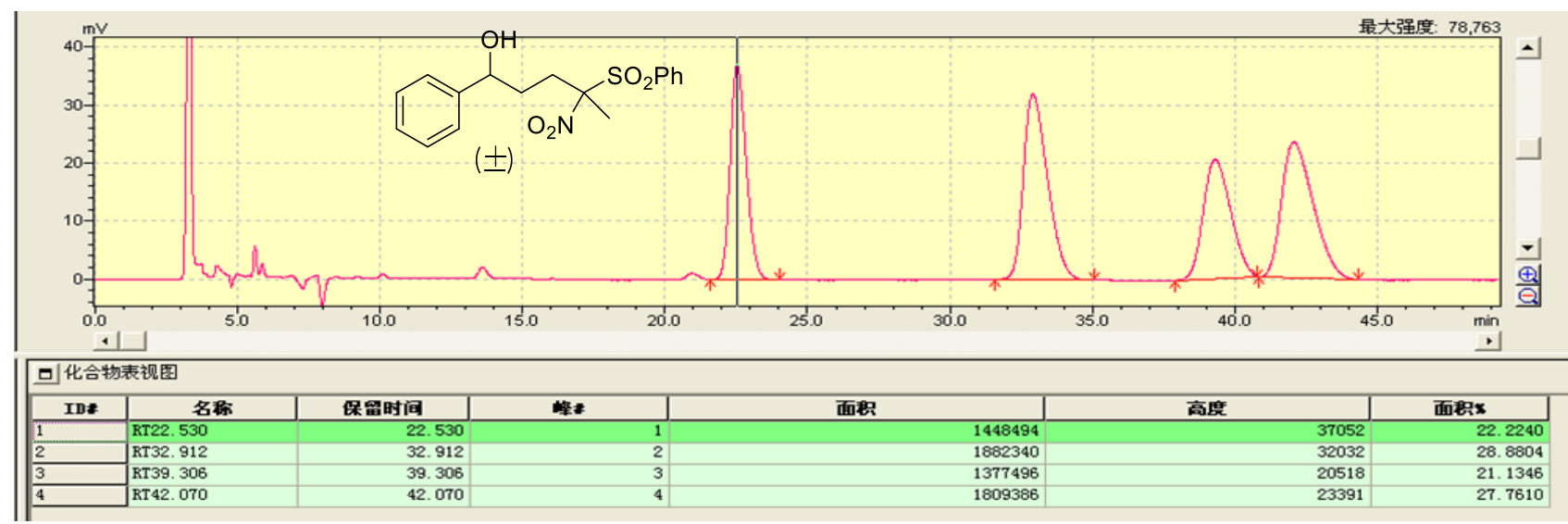

Recycle 1 (98\% yield, 99\% ee, and 98:2 dr)

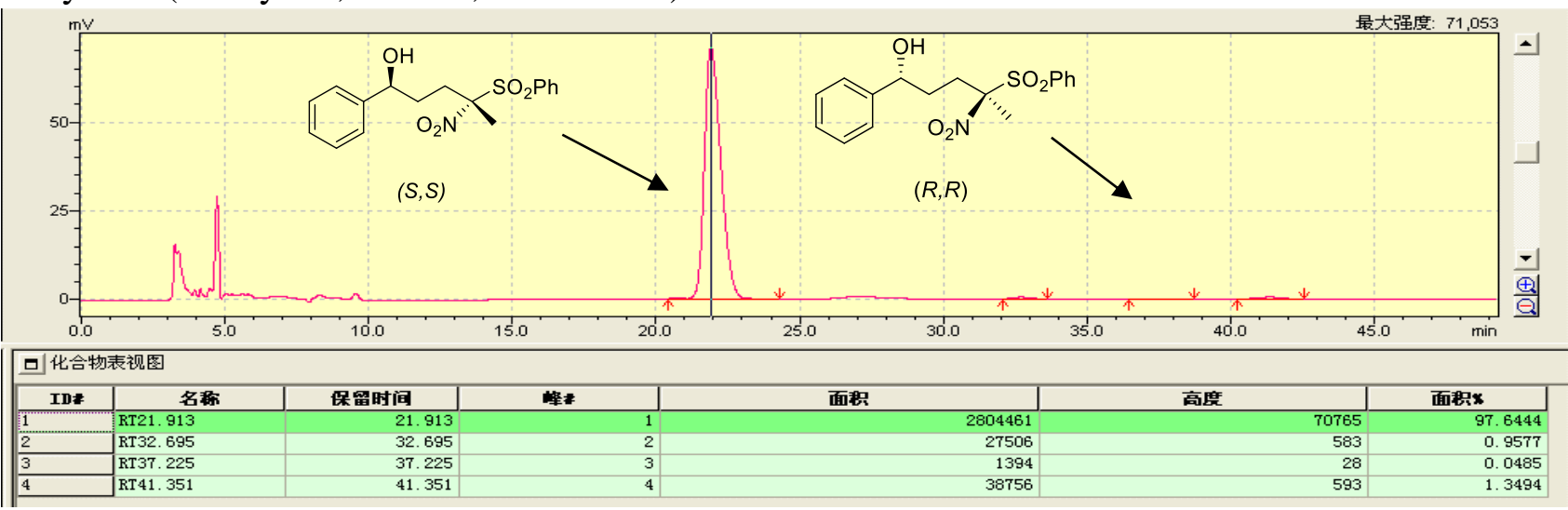


Recycle 2. (97\% yield, 99\% ee, and 98:2 $d r$ )

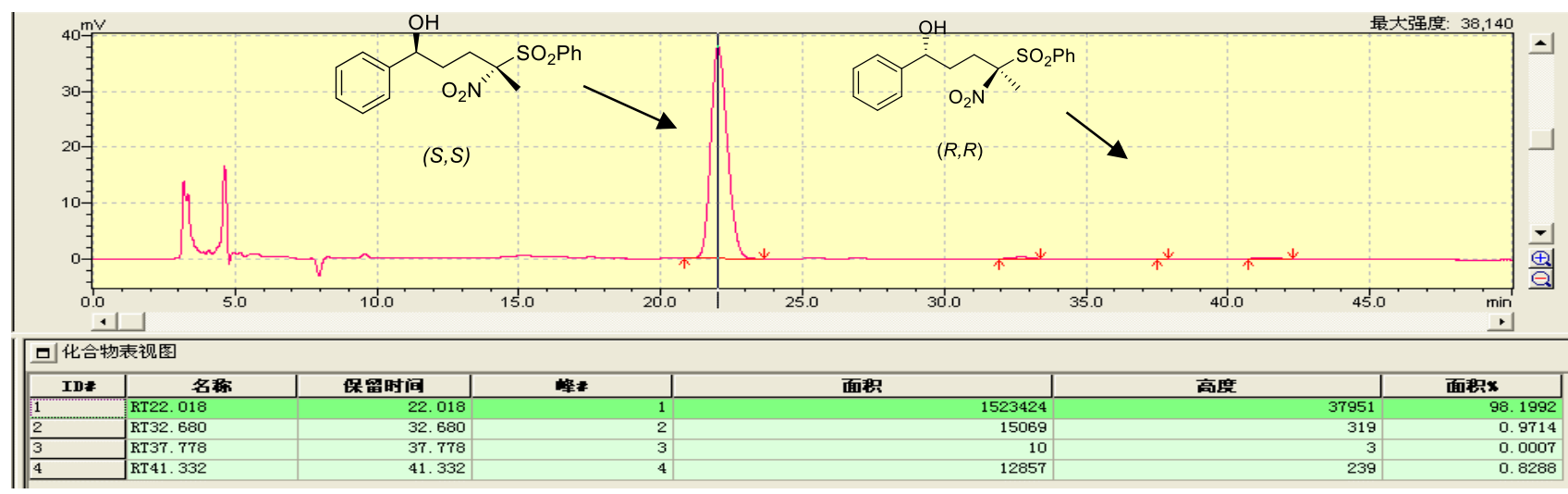

Recycle 3 (97\% yield, 99\% ee, and 98:2 dr)

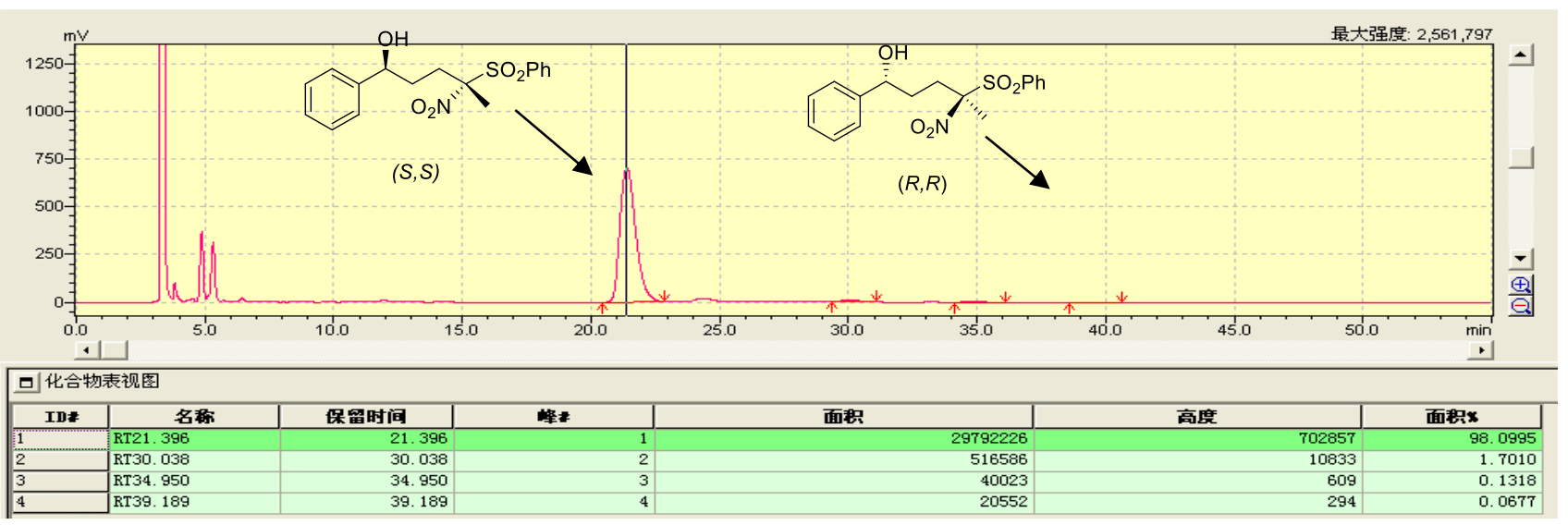

Translation of all characters (Chinese) in above all frameworks to English is as follows:

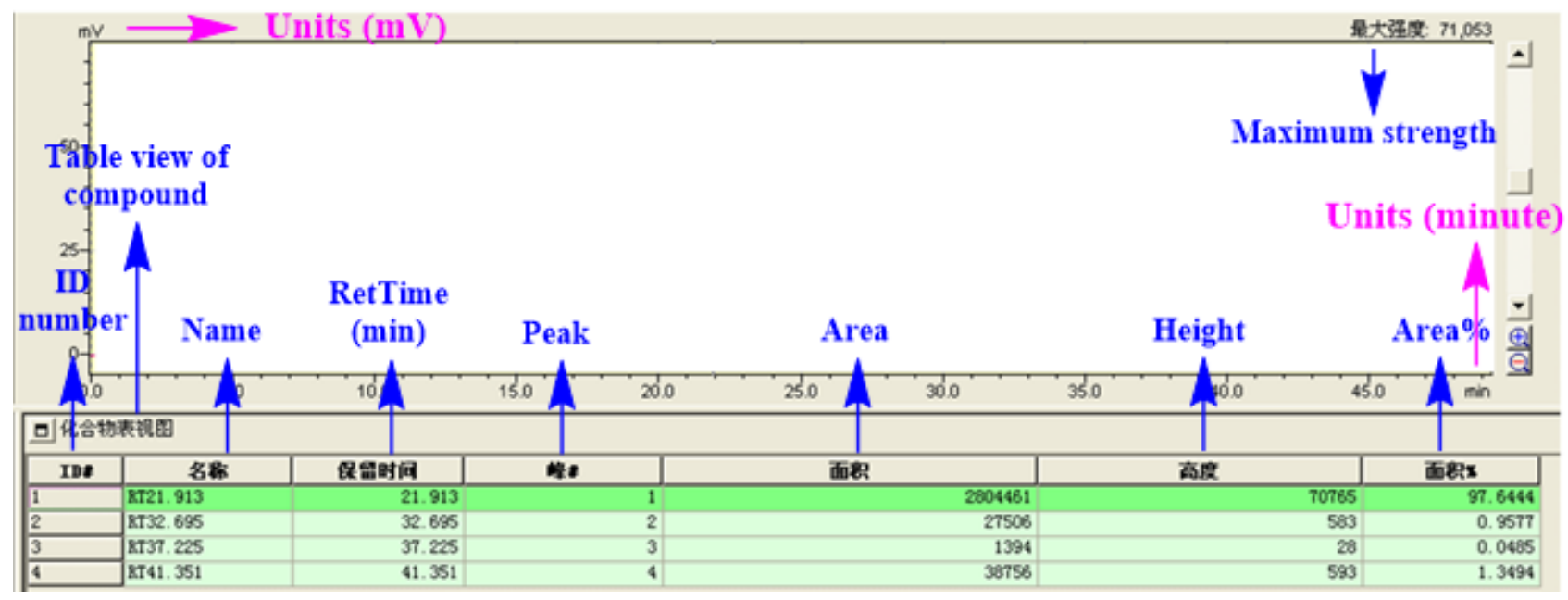


Recycle 4 (96\% yield, 99\% ee, and 98:2 dr)

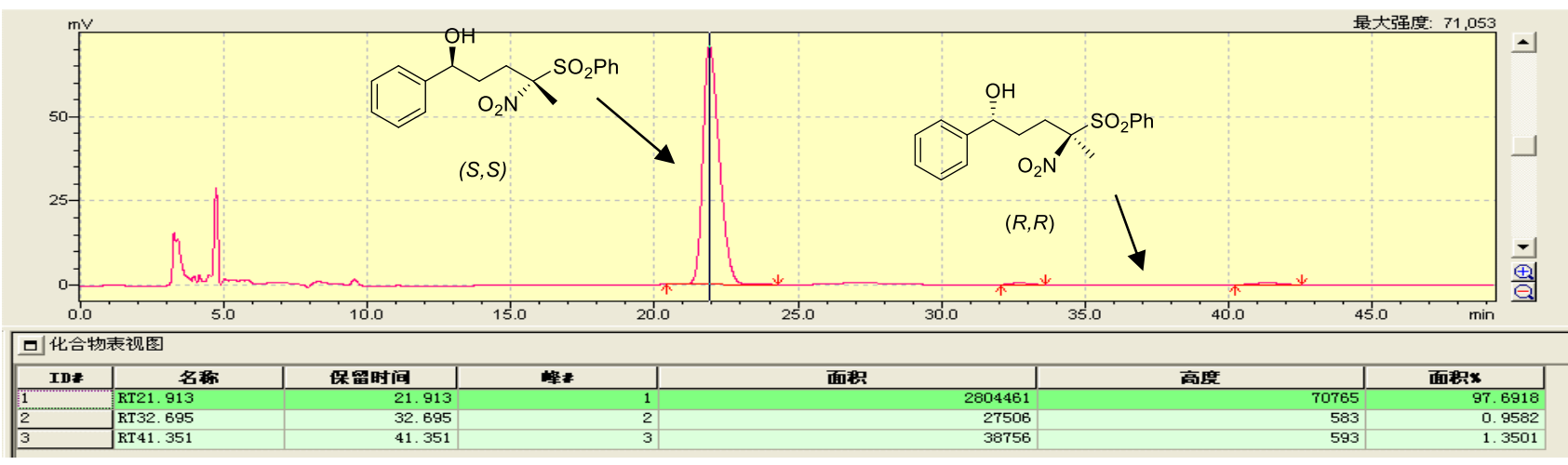

Recycle 5 (96\% yield, 99\% ee, and 97:3 dr)

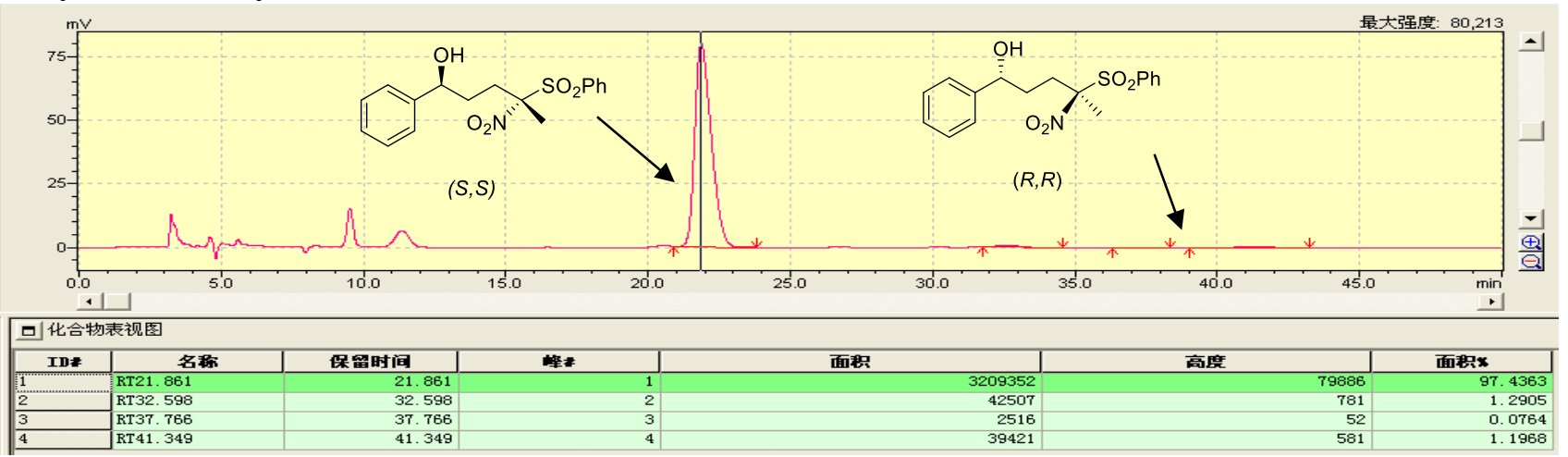

Recycle 6 (95\% yield, 99\% ee, and 94:6 dr)

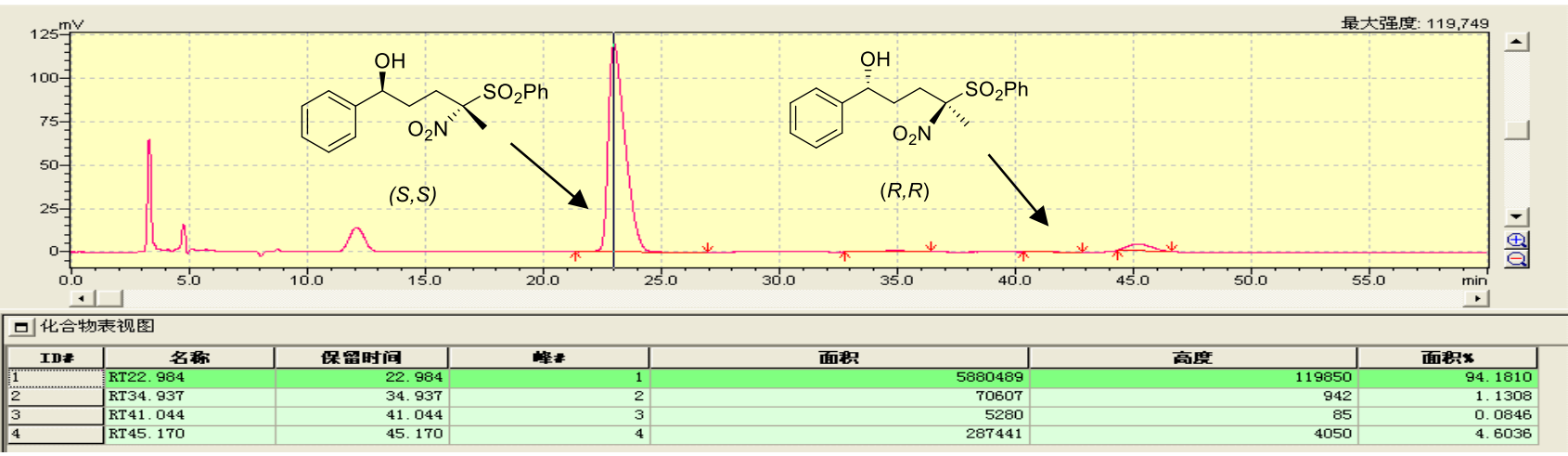

Translation of all characters (Chinese) in above two frameworks to English is as follows:

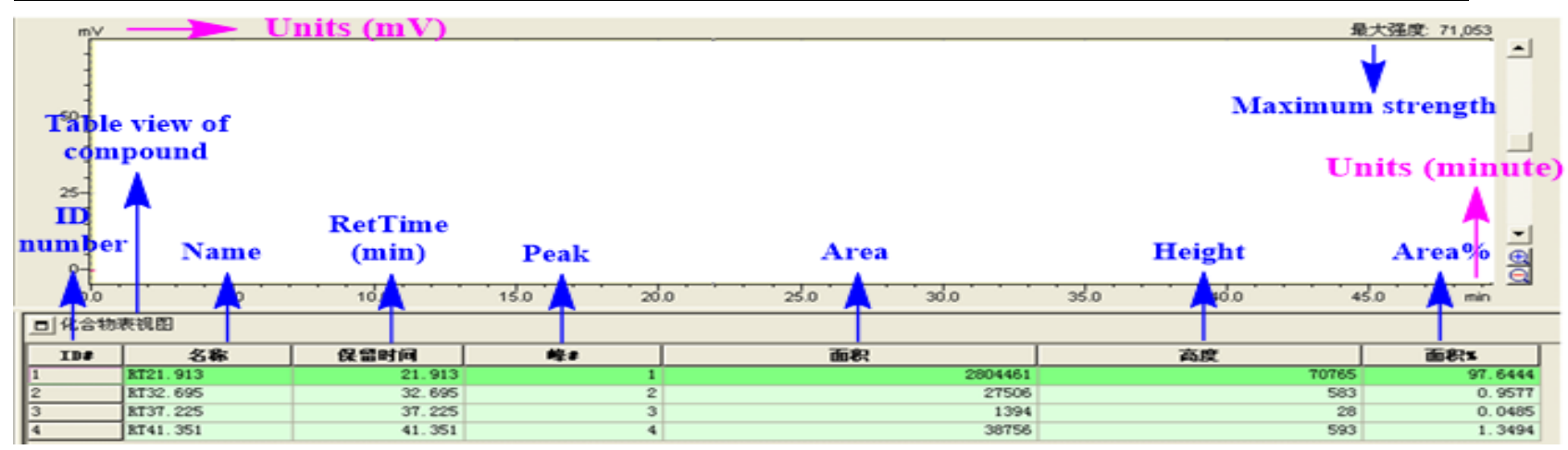

Figure S10. Reusability of co-catalysts in the Michael addition/ATH dual-catalysis process of ((1nitroethyl)sulfonyl)benzene and 1-phenylprop-2-en-1-one. 
8a: (1S,4S)-4-nitro-1-phenyl-4-(phenylsulfonyl)pentan-1-ol.

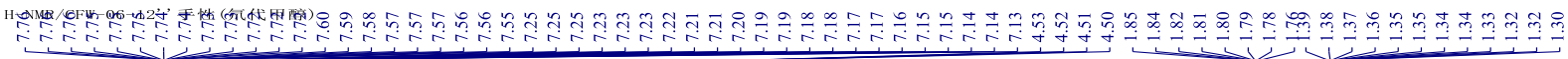
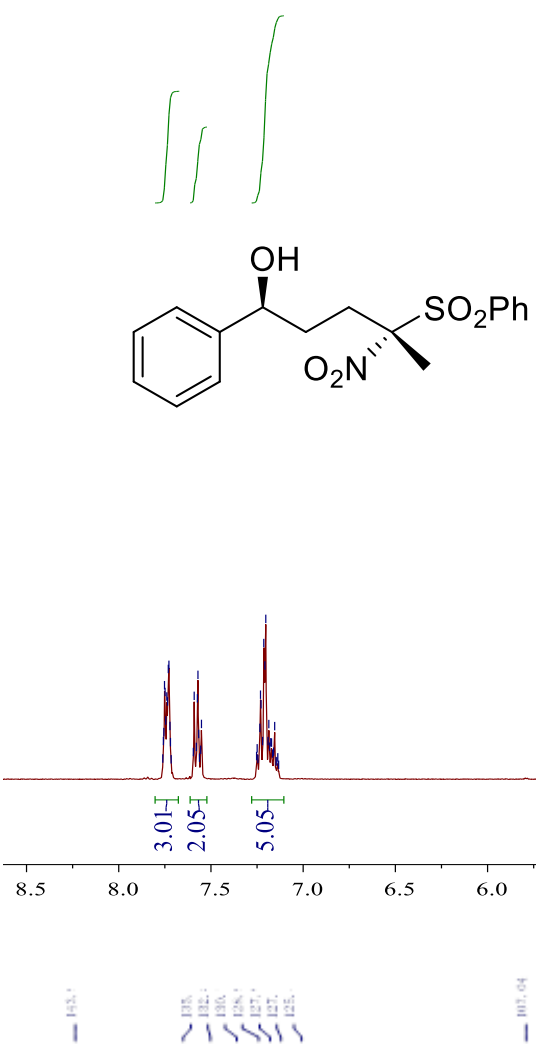

$\int$

坚
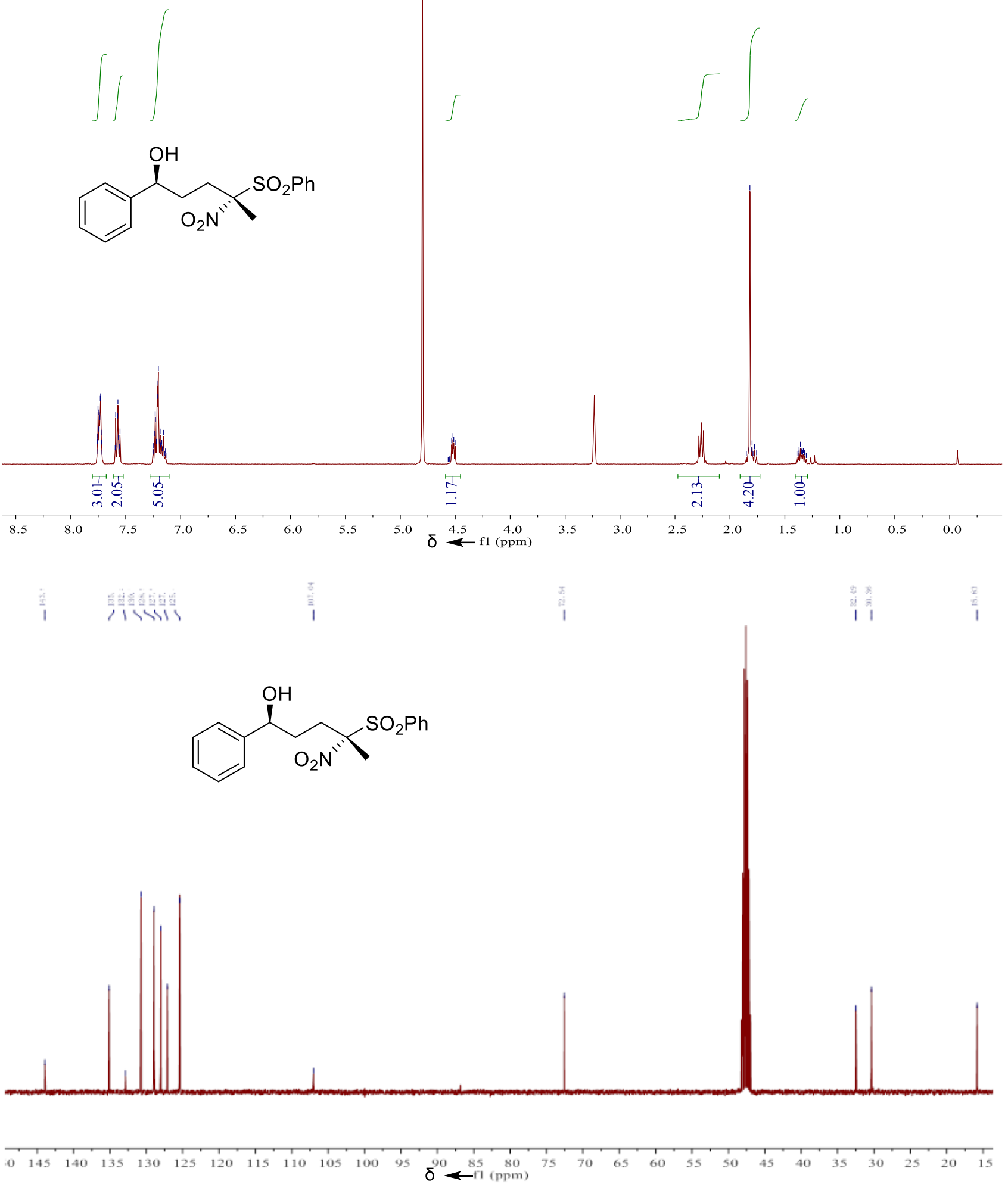

S40 
8b: (1S,4S)-1-(3-fluorophenyl)-4-nitro-4-(phenylsulfonyl)pentan-1-ol.
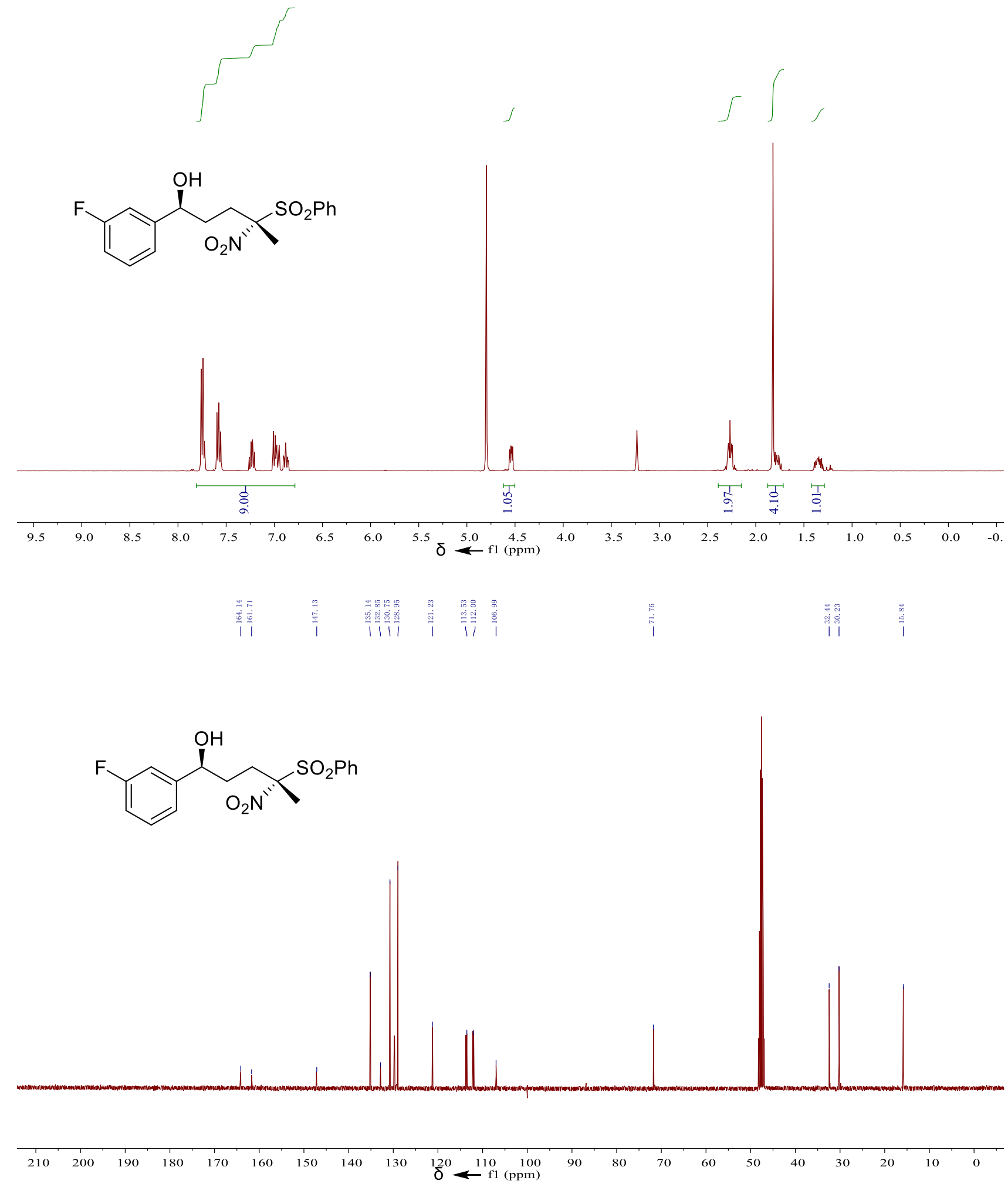

S41 
8c: (1S,4S)-1-(2-fluorophenyl)-4-nitro-4-(phenylsulfonyl)pentan-1-ol.
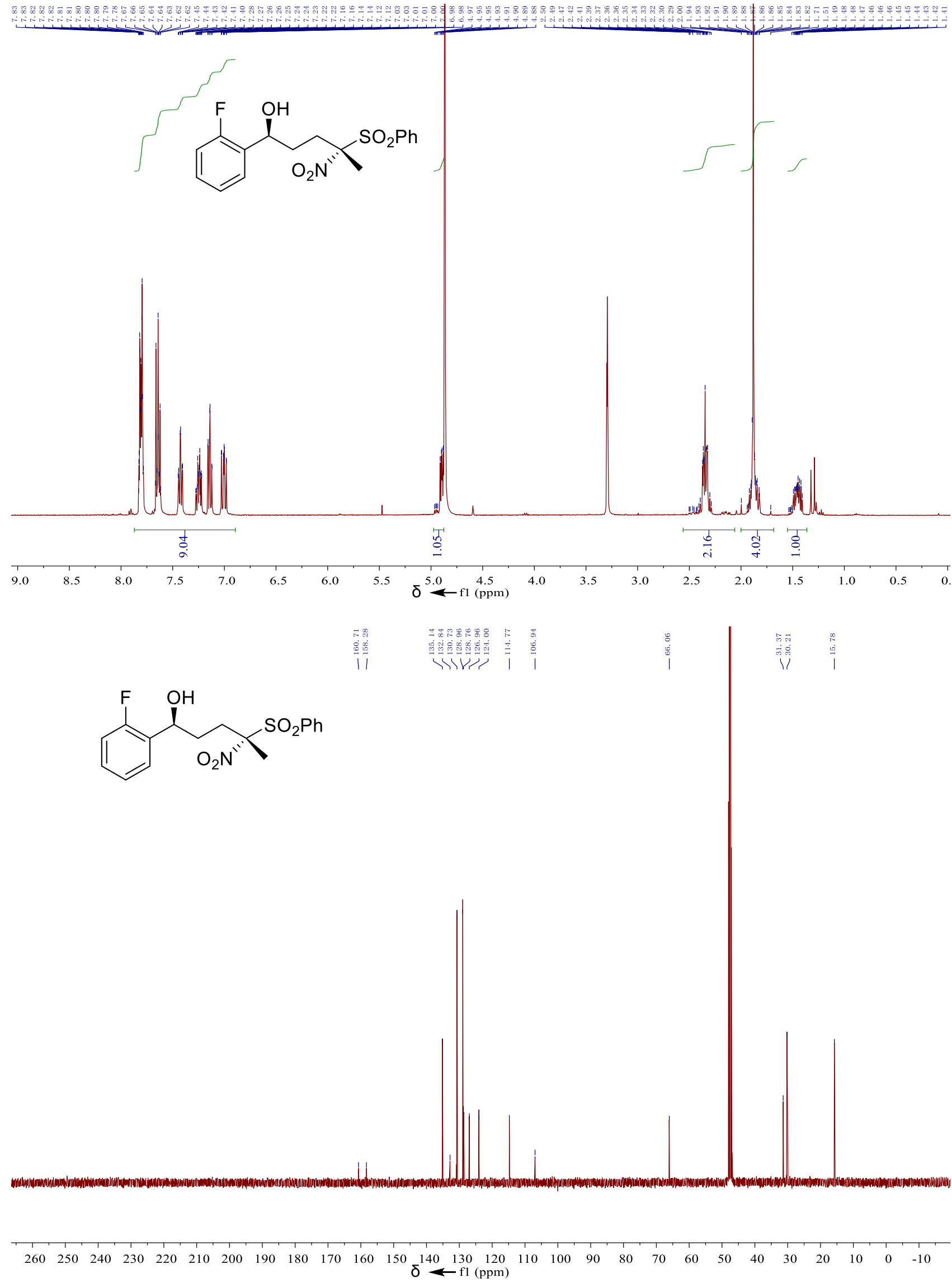
8d:(1S,4S)-1-(4-chlorophenyl)-4-nitro-4-(phenylsulfonyl)pentan-1-ol.

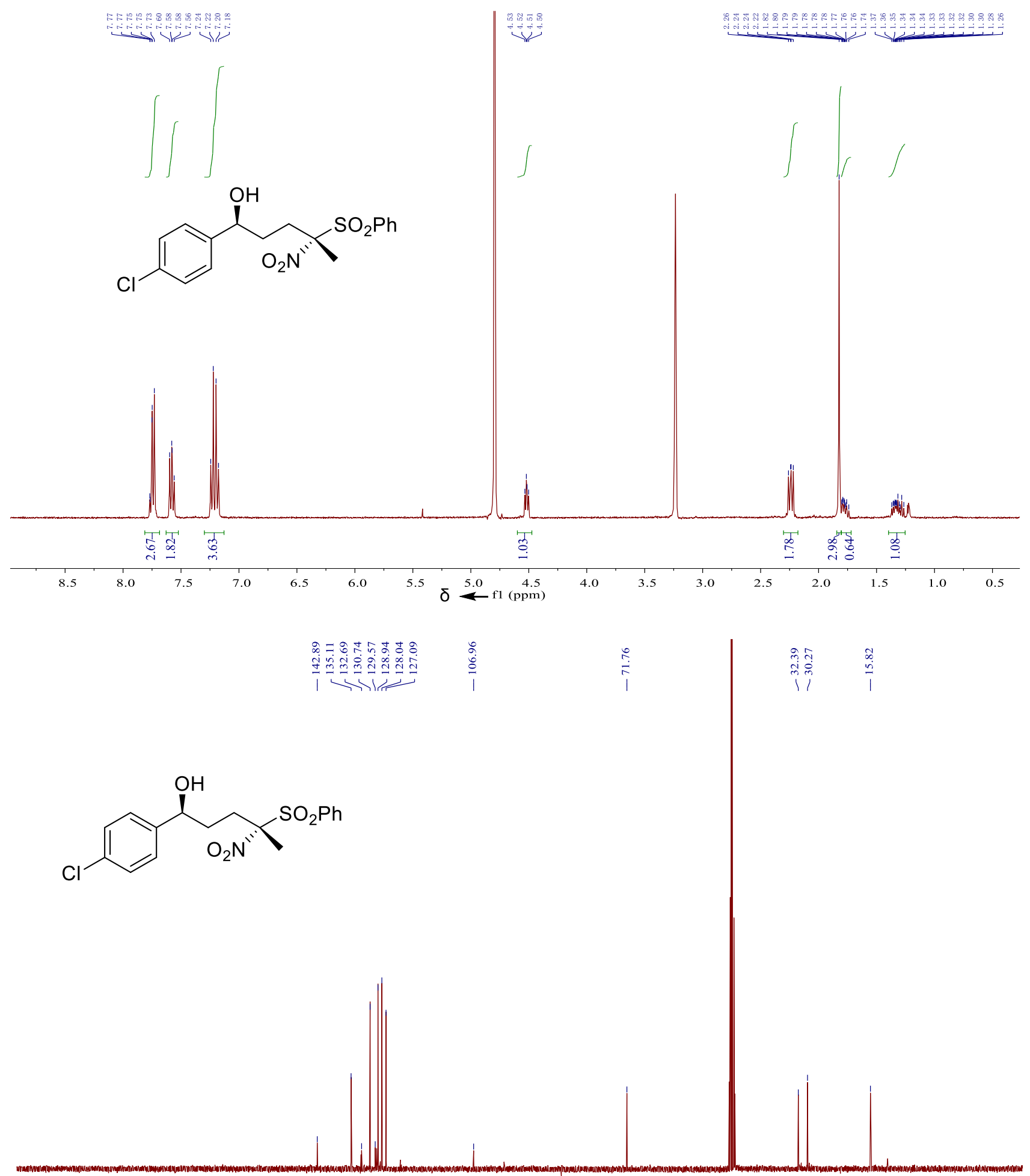

$\begin{array}{llllllllllllllllllllllllll}210 & 200 & 190 & 180 & 170 & 160 & 150 & 140 & 130 & 120 & 110 & 100 & 90 & 80 & 70 & 60 & 50 & 40 & 30 & 20 & 10 & 0 & -10\end{array}$ 
8e: (1S,4S)-1-(2-chlorophenyl)-4-nitro-4-(phenylsulfonyl)pentan-1-ol.

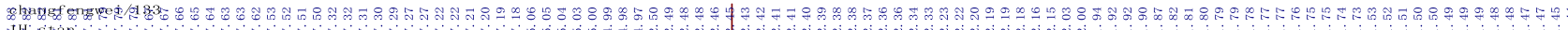

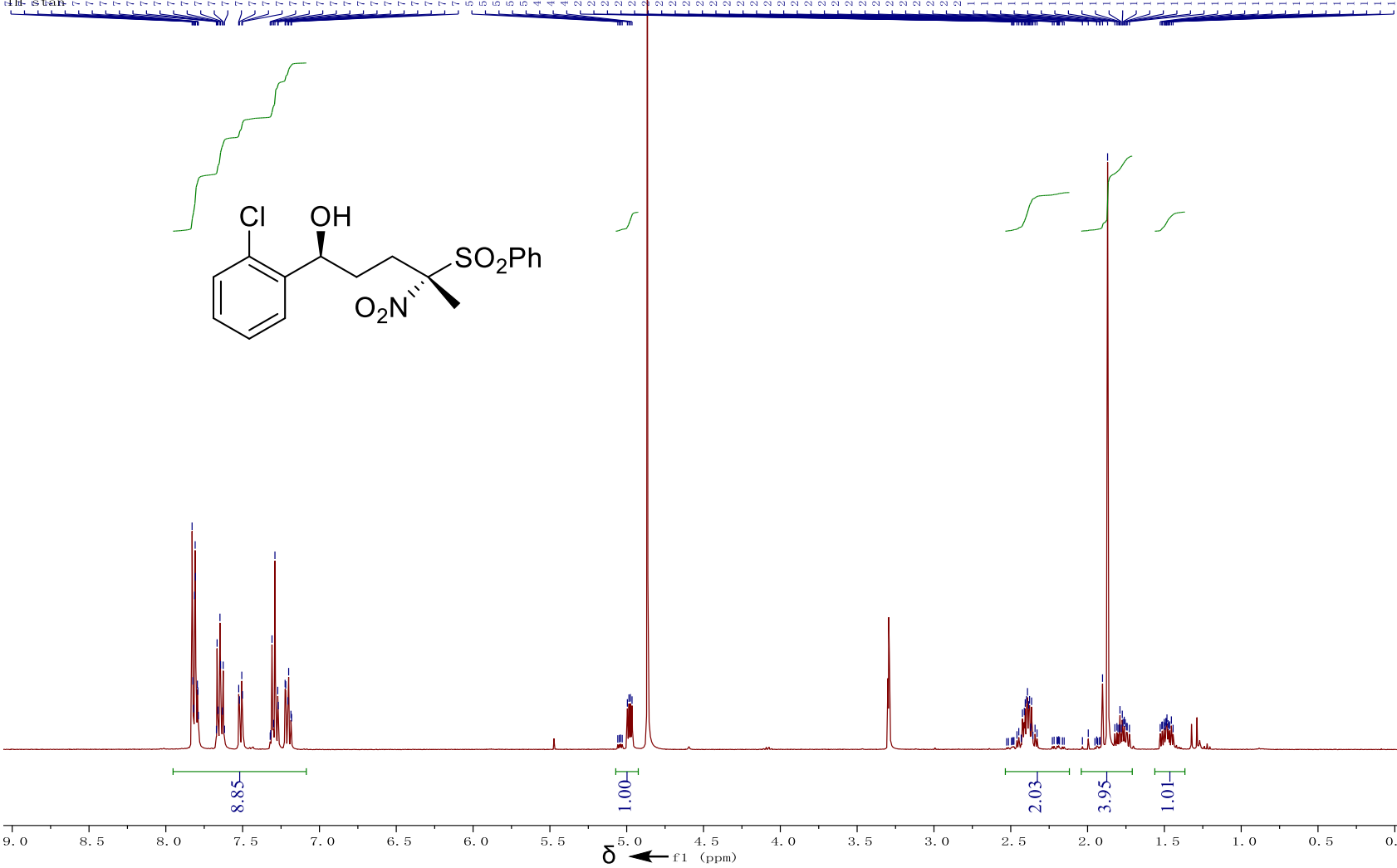

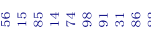

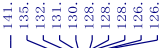

\begin{tabular}{l}
8 \\
8 \\
$\stackrel{8}{\circ}$ \\
\hline
\end{tabular}

$\underbrace{\mathrm{Cl}}_{\mathrm{O}_{2} \mathrm{~N}} \mathrm{SO}_{2}^{\mathrm{PH}}$

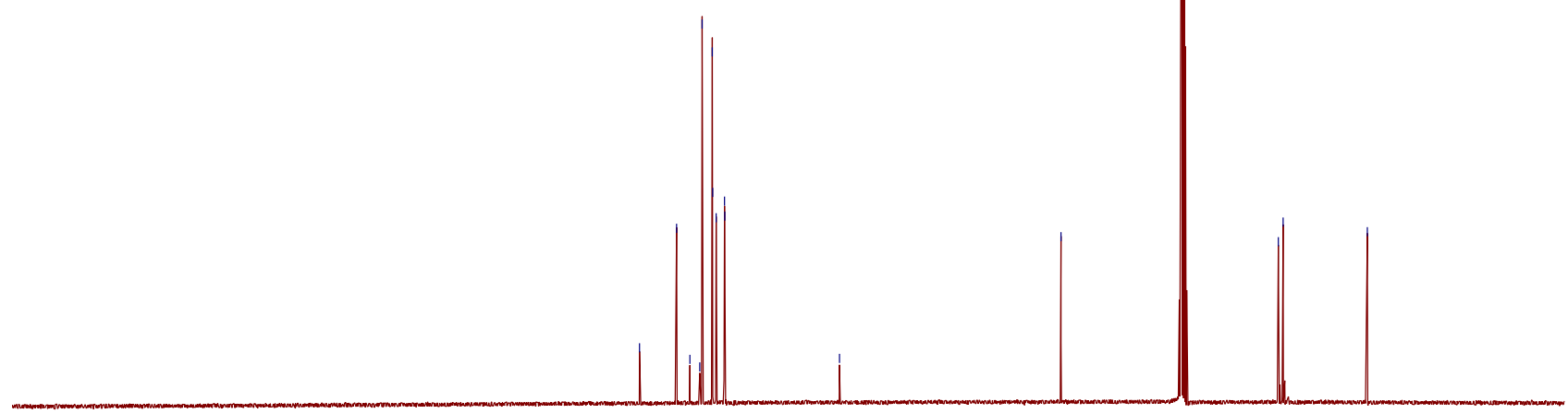

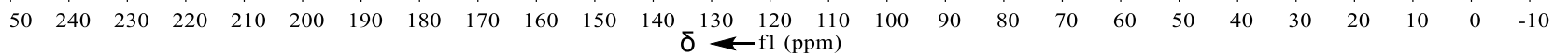


8f: (1S,4S)-1-(4-bromophenyl)-4-nitro-4-(phenylsulfonyl)pentan-1-ol.

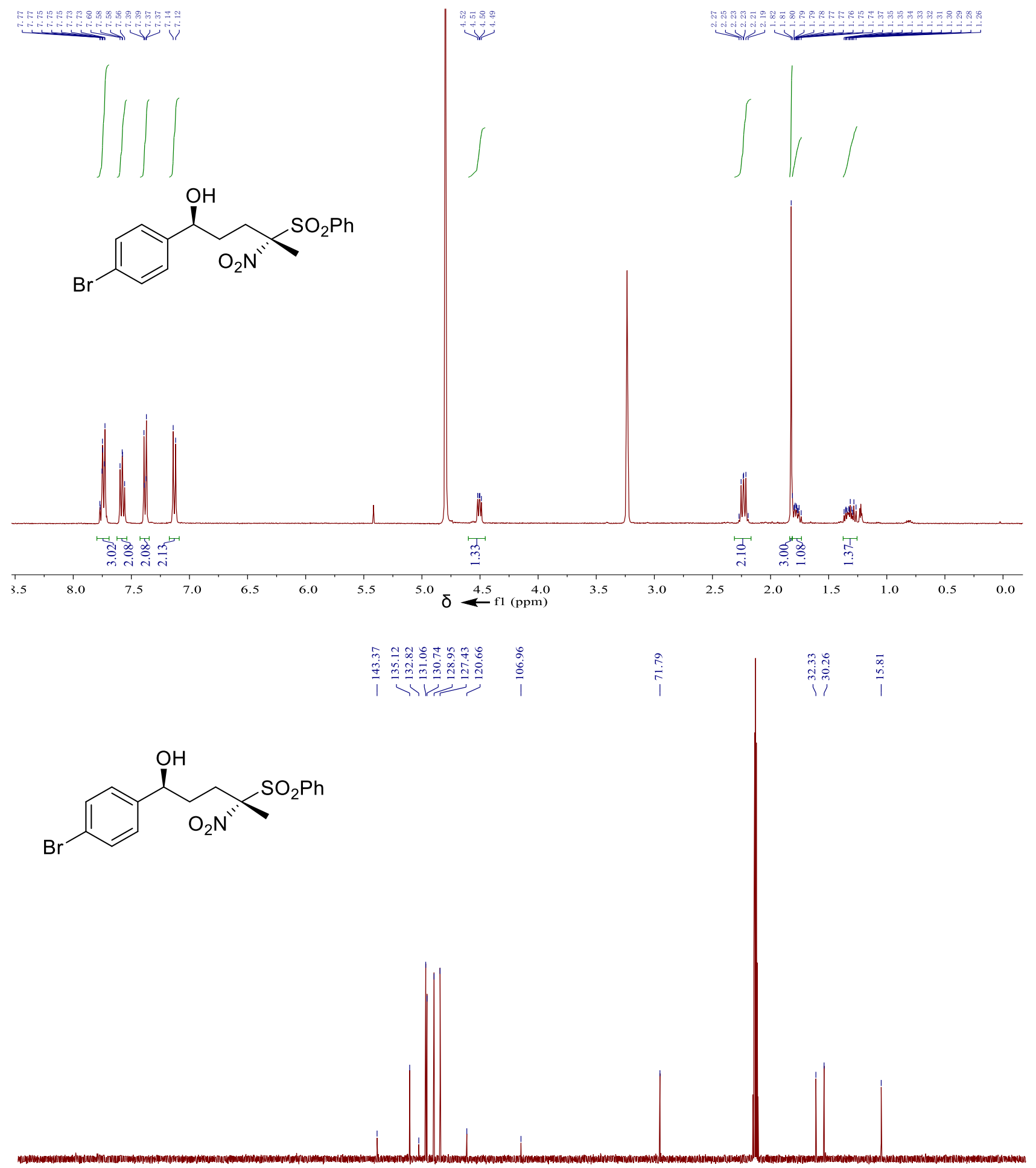

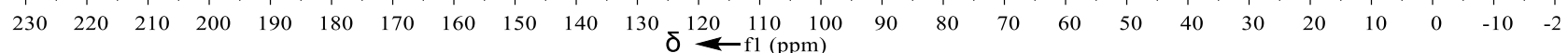


8g: (1S,4S)-1-(3-bromophenyl)-4-nitro-4-(phenylsulfonyl)pentan-1-ol.
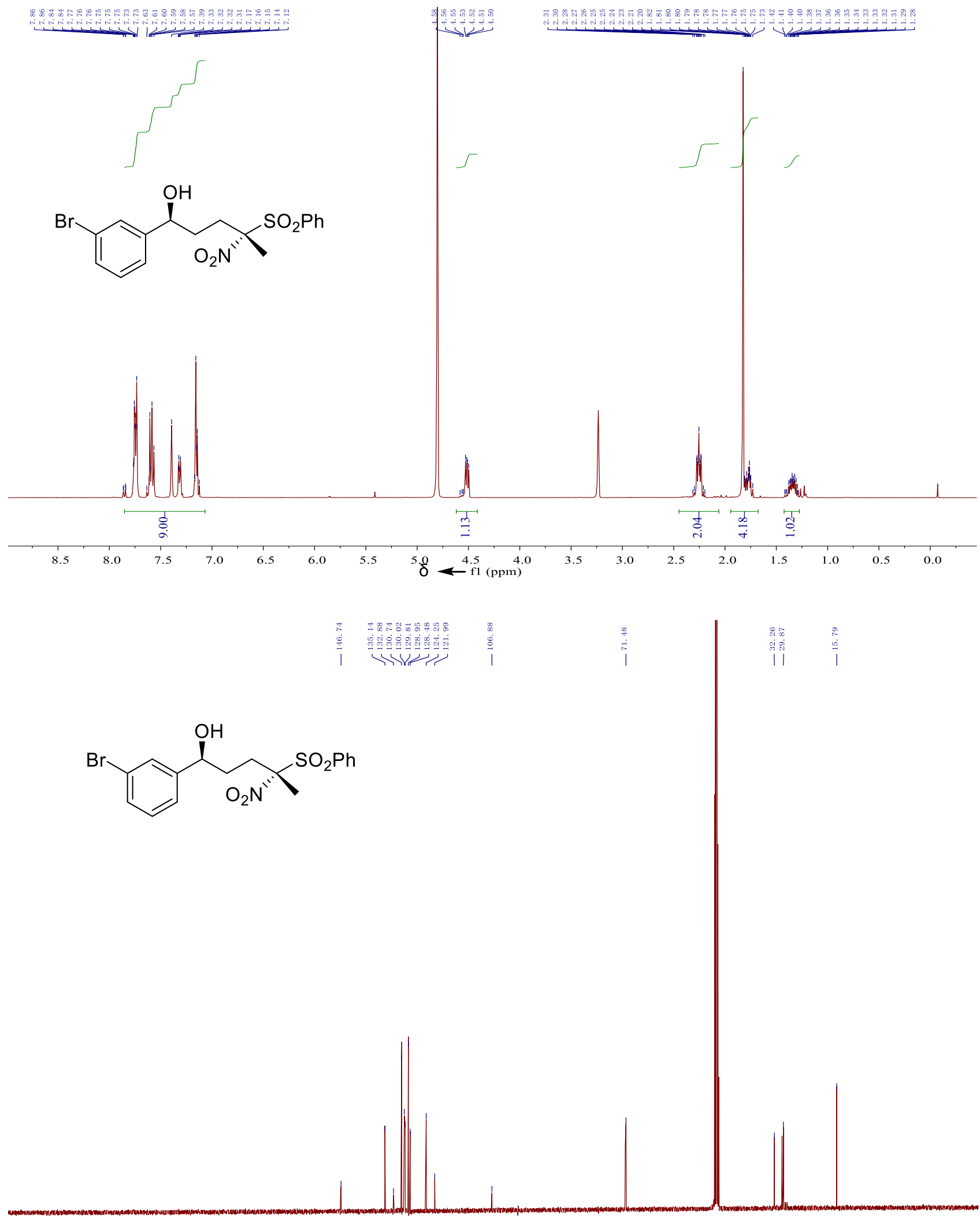

$\begin{array}{llllllllllllllllllllllllllllllllll}230 & 220 & 210 & 200 & 190 & 180 & 170 & 160 & 150 & 140 & 130 & 120 & 110 & 100 & 90 & 80 & 70 & 60 & 50 & 40 & 30 & 20 & 10 & 0 & -10 & -2\end{array}$ 
8h: (1S,4S)-1-(4-iodophenyl)-4-nitro-4-(phenylsulfonyl)pentan-1-ol.
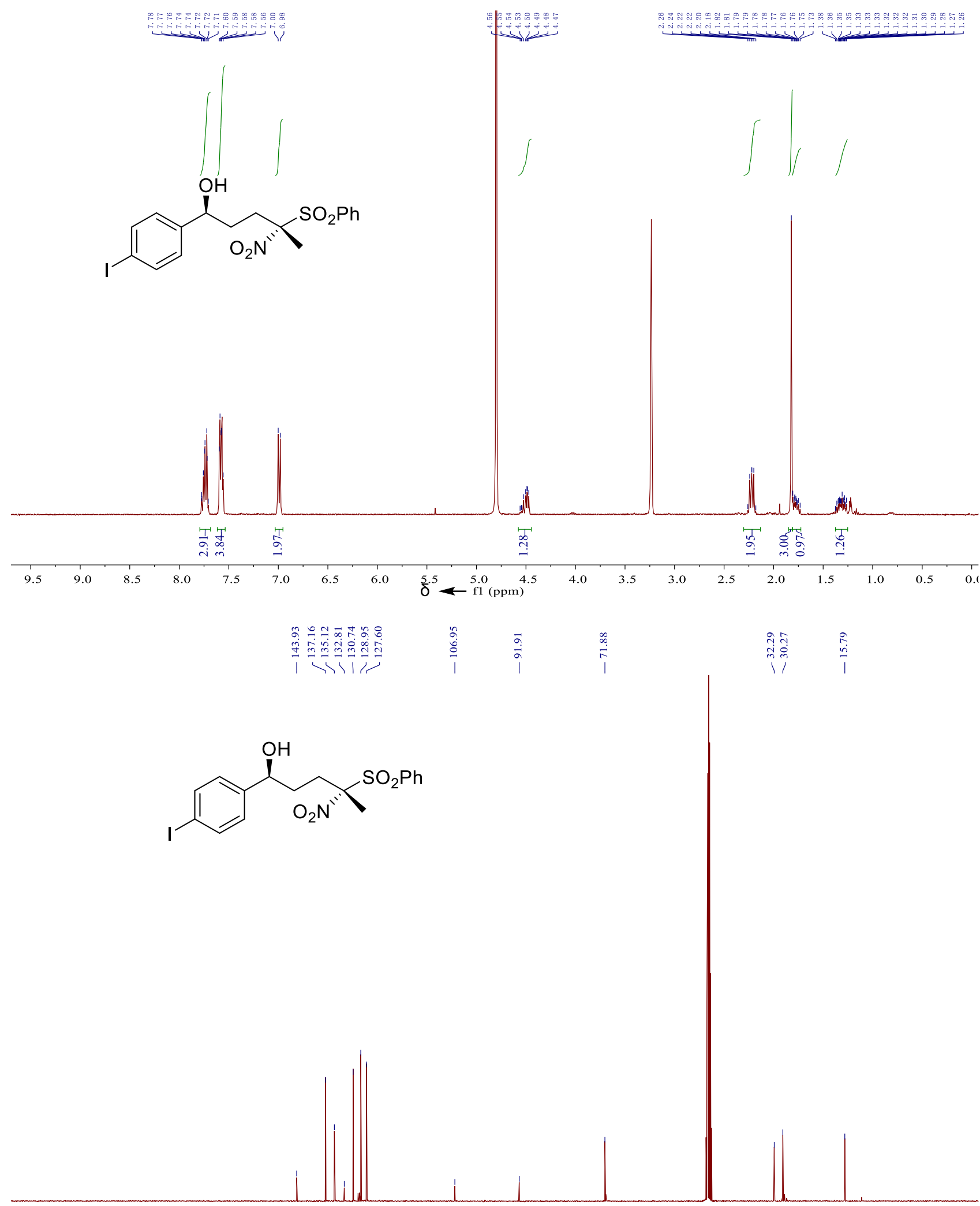

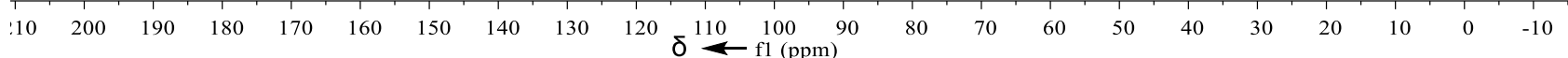


8i: (1S,4S)-4-nitro-1-(4-nitrophenyl)-4-(phenylsulfonyl)pentan-1-ol.

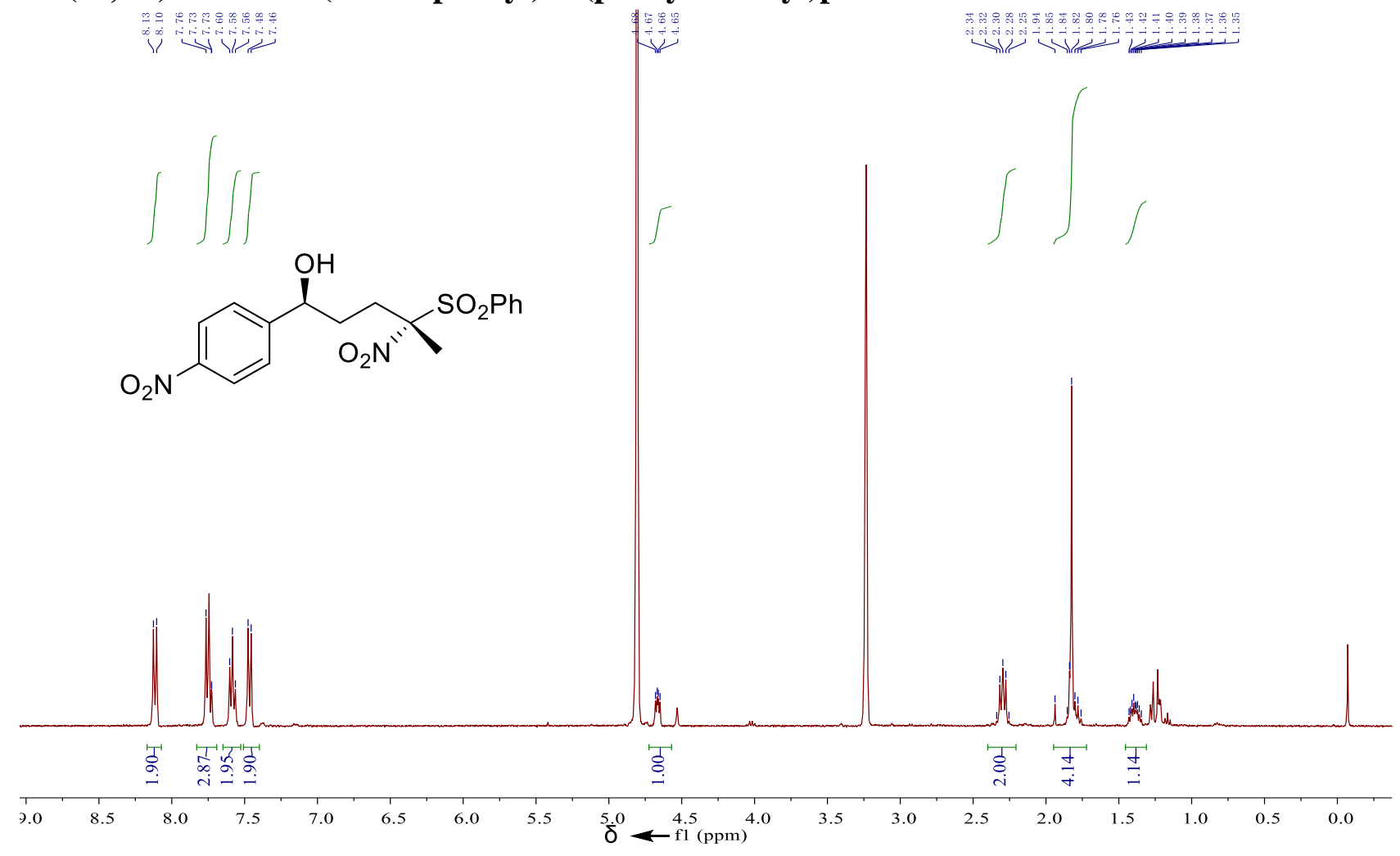<smiles>C[C@](CC[C@H](O)c1ccc([N+](=O)[O-])cc1)(Oc1ccccc1)[N+](=O)[O-]</smiles>

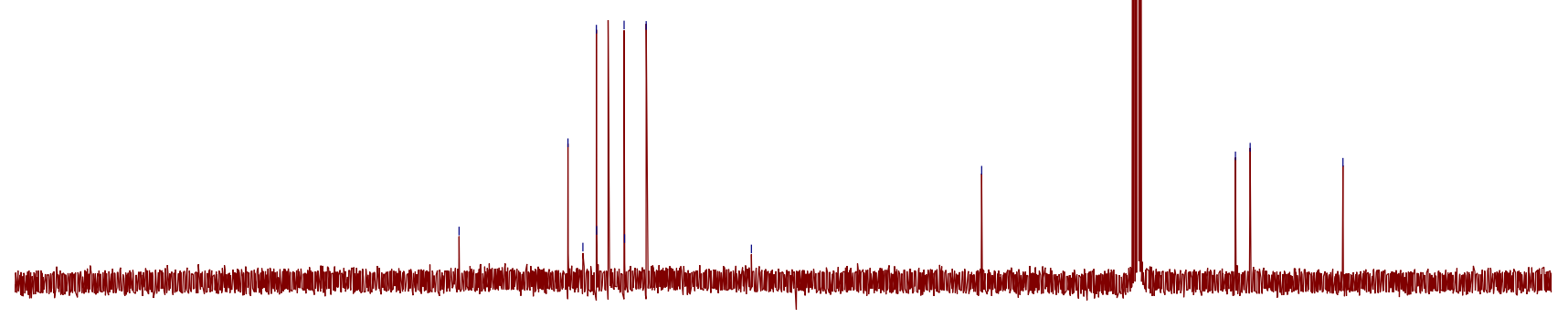
$\begin{array}{llllllllllllllllllllllllllll}20 & 210 & 200 & 190 & 180 & 170 & 160 & 150 & 140 & 130 & 120 & 110 & 100 & 90 & 80 & 70 & 60 & 50 & 40 & 30 & 20 & 10 & 0 & -10\end{array}$ 
8j: 4-((1S,4S)-1-hydroxy-4-nitro-4-(phenylsulfonyl)pentyl)benzonitrile.

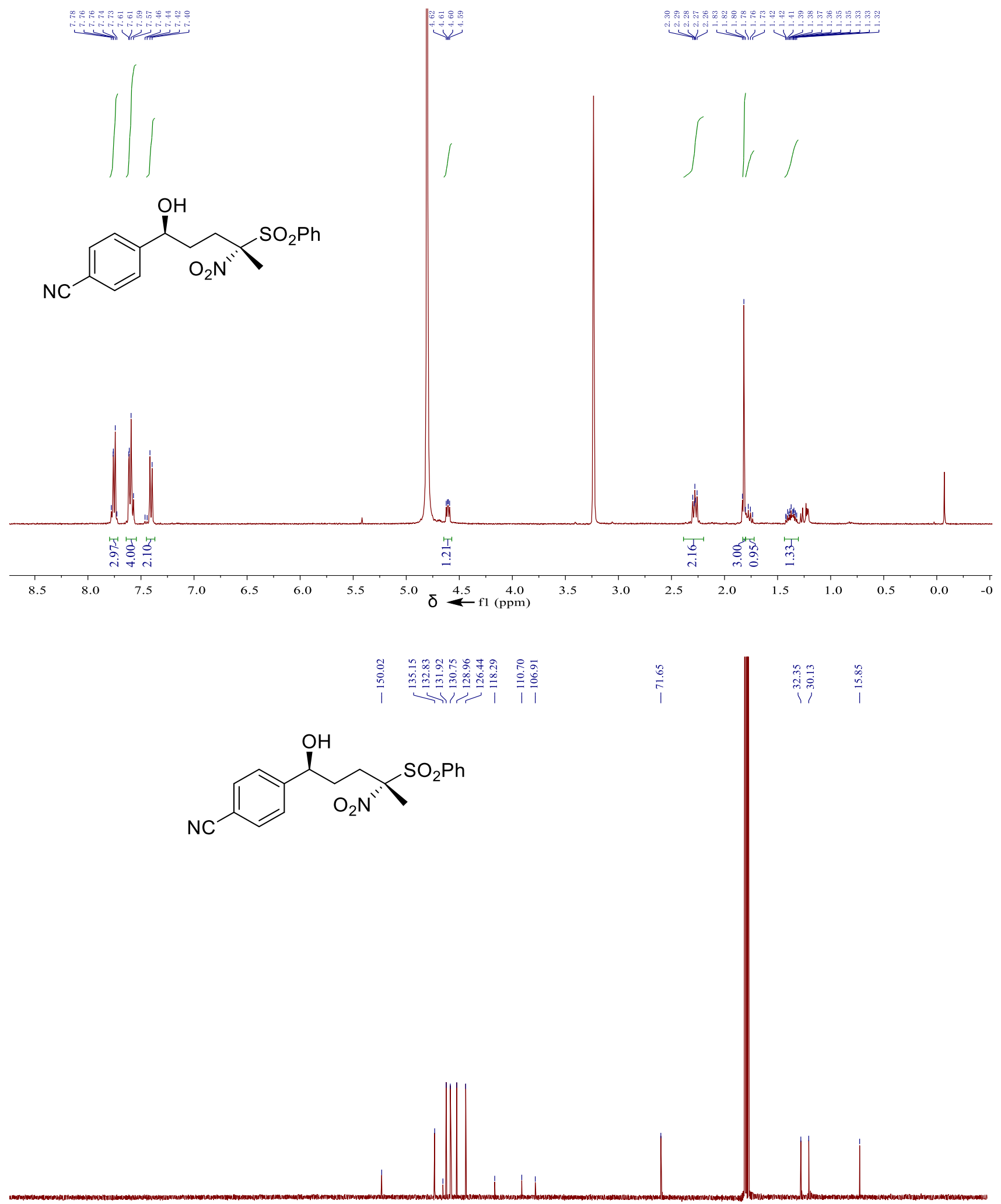

$\begin{array}{llllllllllllllllllllllllllllllll}250 & 240 & 230 & 220 & 210 & 200 & 190 & 180 & 170 & 160 & 150 & 140 & 130 & 120 & 110 & 100 & 90 & 80 & 70 & 60 & 50 & 40 & 30 & 20 & 10 & 0 & -10 & -\therefore\end{array}$ 
8k: (1S,4S)-1-(3-methoxyphenyl)-4-nitro-4-(phenylsulfonyl)pentan-1-ol.

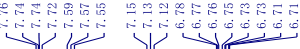

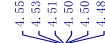

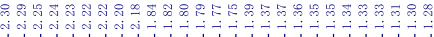<smiles>COc1cccc([C@@H](O)CC[C@](C)(O)[N+](=O)[O-])c1</smiles>

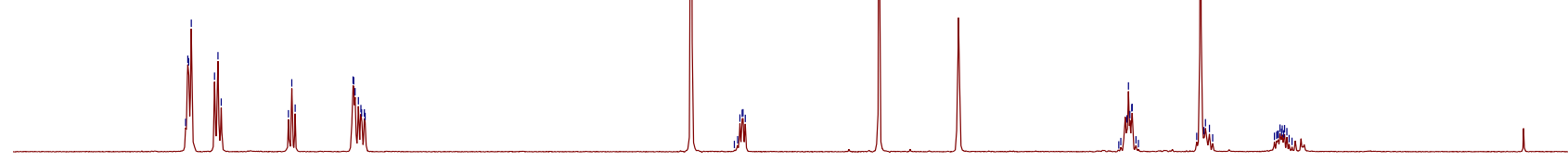

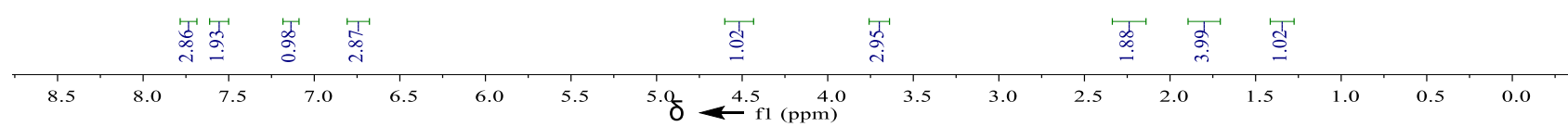

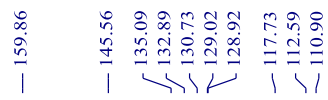<smiles>COc1cccc(C(O)CCC(C)([N+](=O)[O-])S(=O)(=O)c2ccccc2)c1</smiles>

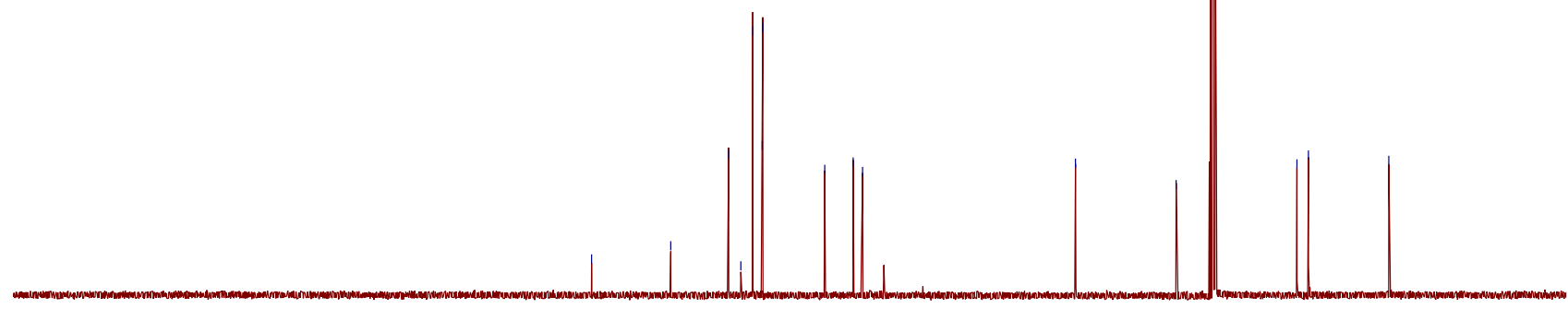

$\begin{array}{lllllllllllllllllllllllllllllll}260 & 250 & 240 & 230 & 220 & 210 & 200 & 190 & 180 & 170 & 160 & 150 & 140 & 130 & 120 & 110 & 100 & 90 & 80 & 70 & 60 & 50 & 40 & 30 & 20 & 10 & 0 & -10\end{array}$ 
81: (1S,4S)-1-(4-methoxyphenyl)-4-nitro-4-(phenylsulfonyl)pentan-1-ol.

等
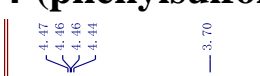

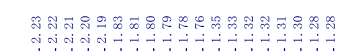

$\underbrace{2}$<smiles>COc1ccc([C@@H](O)CC[C@](C)(O[R6](=O)O)[N+](=O)[O-])cc1</smiles><smiles>CCCC[C@@](C)(CC[C@@H](O)c1ccc(OC)cc1)[N+](=O)[O-]</smiles>

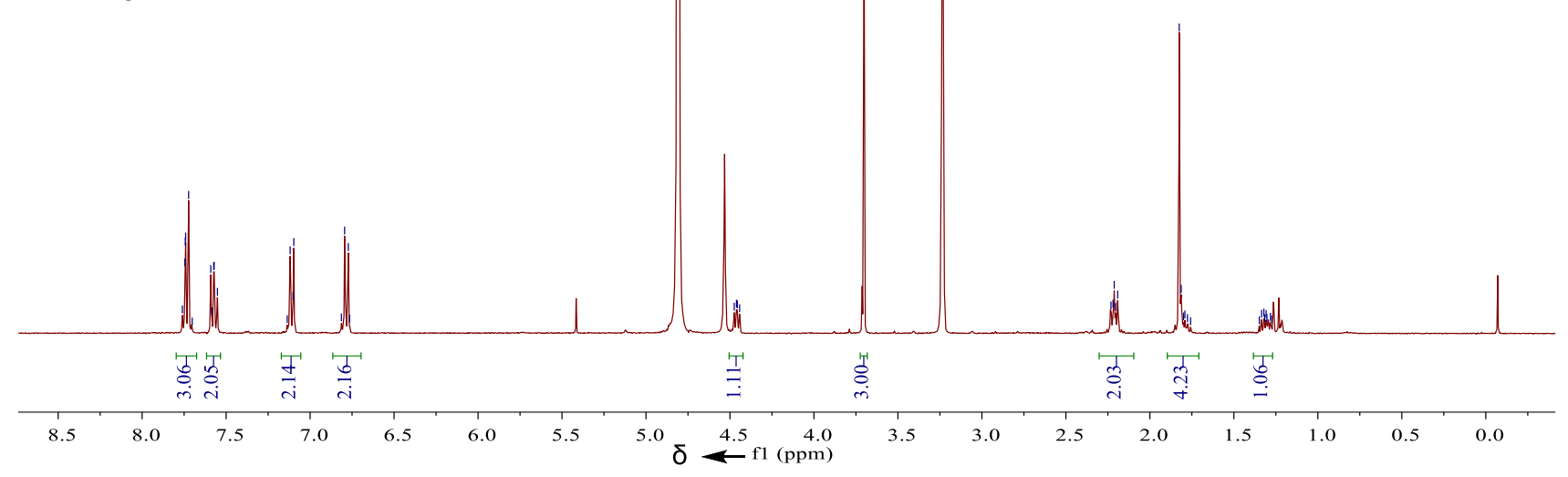

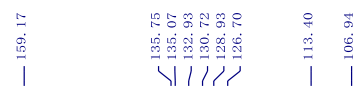<smiles>COc1ccc([C@H](O)CC[C@](C)(OS(=O)(=O)c2ccccc2)[N+](=O)[O-])cc1</smiles>

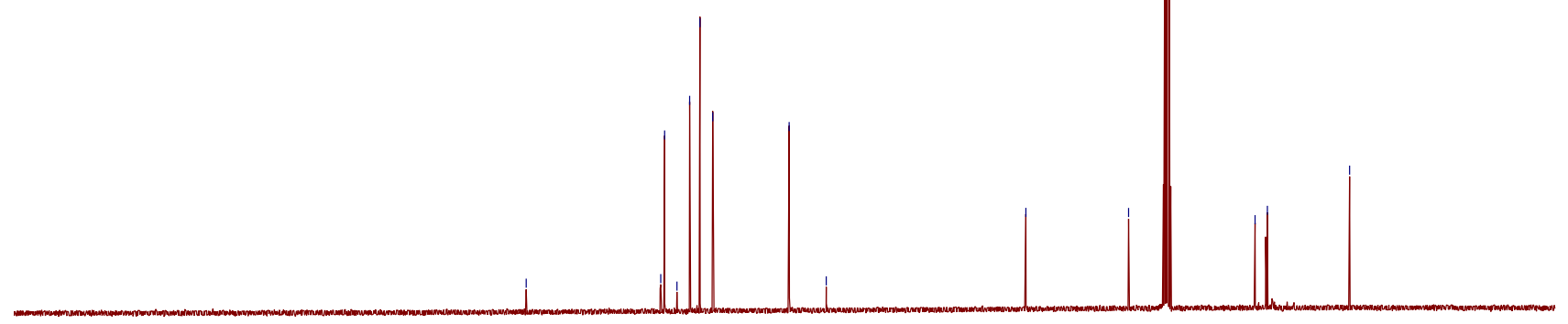

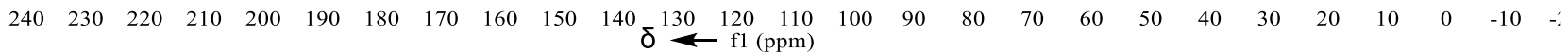


8m: (1S,4S)-4-nitro-4-(phenylsulfonyl)-1-(p-tolyl)pentan-1-ol.

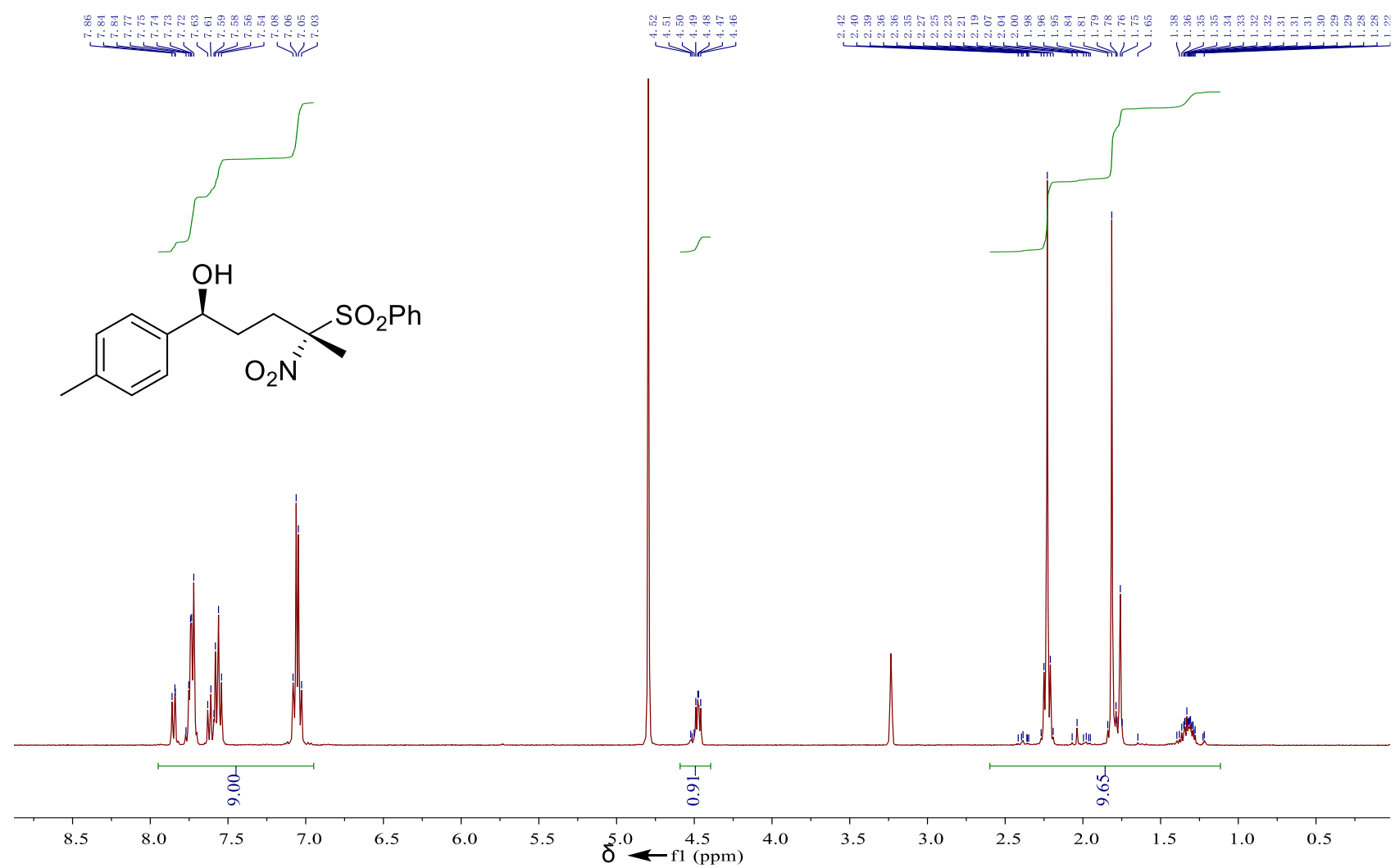

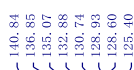

|रा11V।

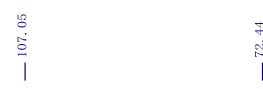

$\frac{\sqrt[4]{1}}{\sqrt[4]{4}}$

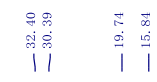<smiles>Cc1ccc([C@H](O)CC[C@](C)([N+](=O)[O-])[Sb](=O)(=O)c2ccccc2)cc1</smiles>

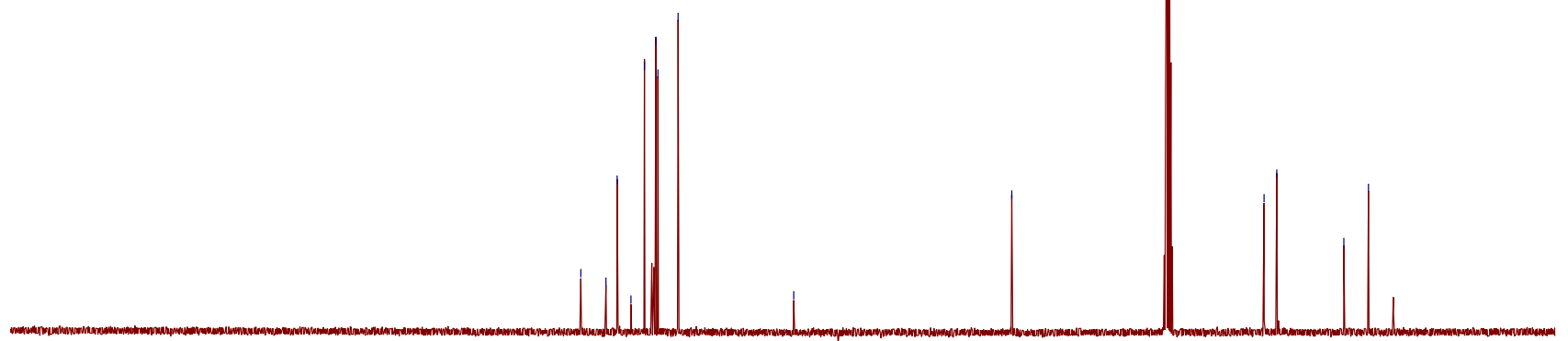

$\begin{array}{llllllllllllllllllllllllll}230 & 220 & 210 & 200 & 190 & 180 & 170 & 160 & 150 & 140 & 130 & 120 & 110 & 100 & 90 & 80 & 70 & 60 & 50 & 40 & 30 & 20 & 10 & 0 & -10\end{array}$ 
8n: (1S,4S)-4-nitro-4-(phenylsulfonyl)-1-(m-tolyl)pentan-1-ol.

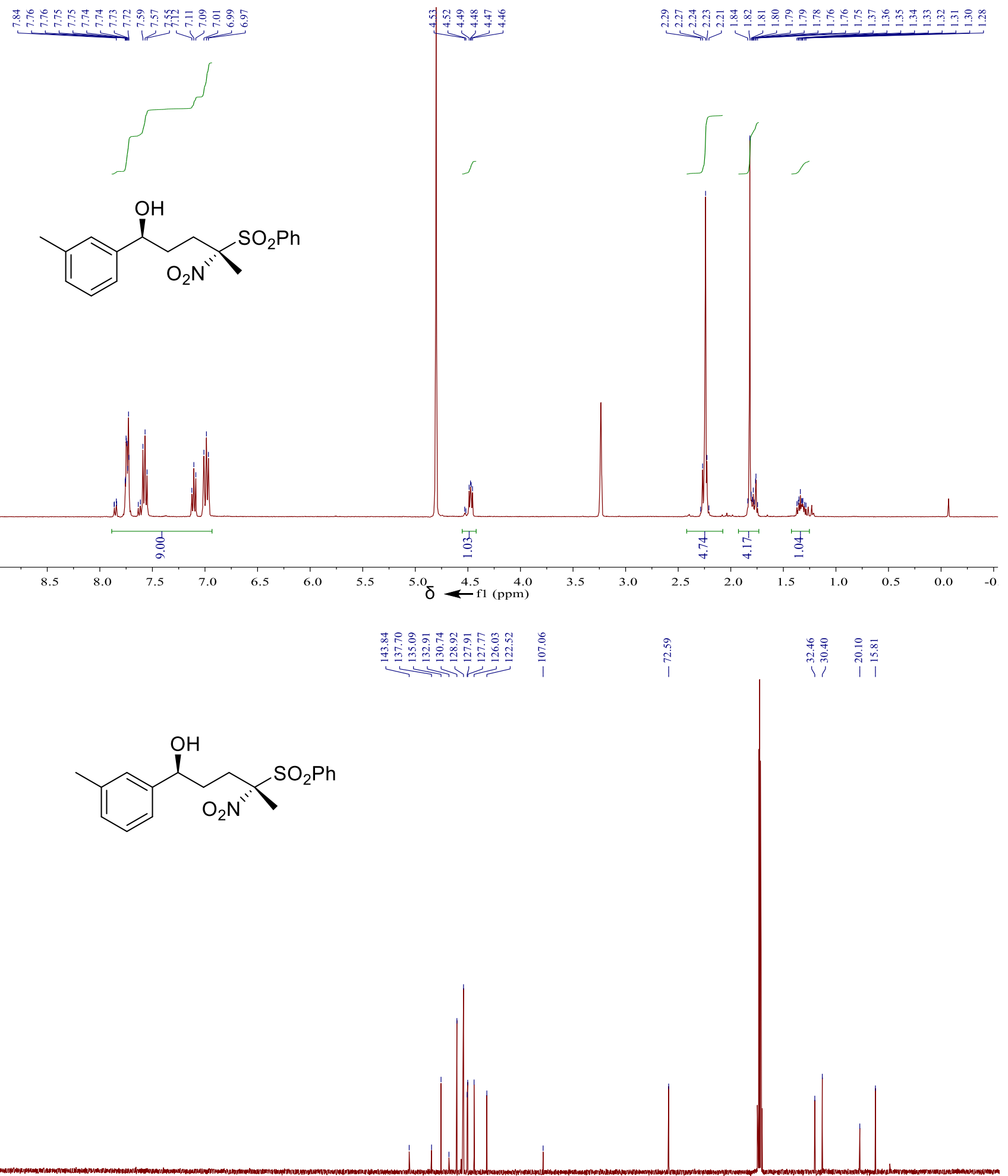

$\begin{array}{llllllllllllllllllllllllllllll}250 & 240 & 230 & 220 & 210 & 200 & 190 & 180 & 170 & 160 & 150 & 140 & 130 & 120 & 110 & 100 & 90 & 80 & 70 & 60 & 50 & 40 & 30 & 20 & 10 & 0 & -10\end{array}$ 
8o: (1S,4S)-1-(naphthalen-2-yl)-4-nitro-4-(phenylsulfonyl)pentan-1-ol.

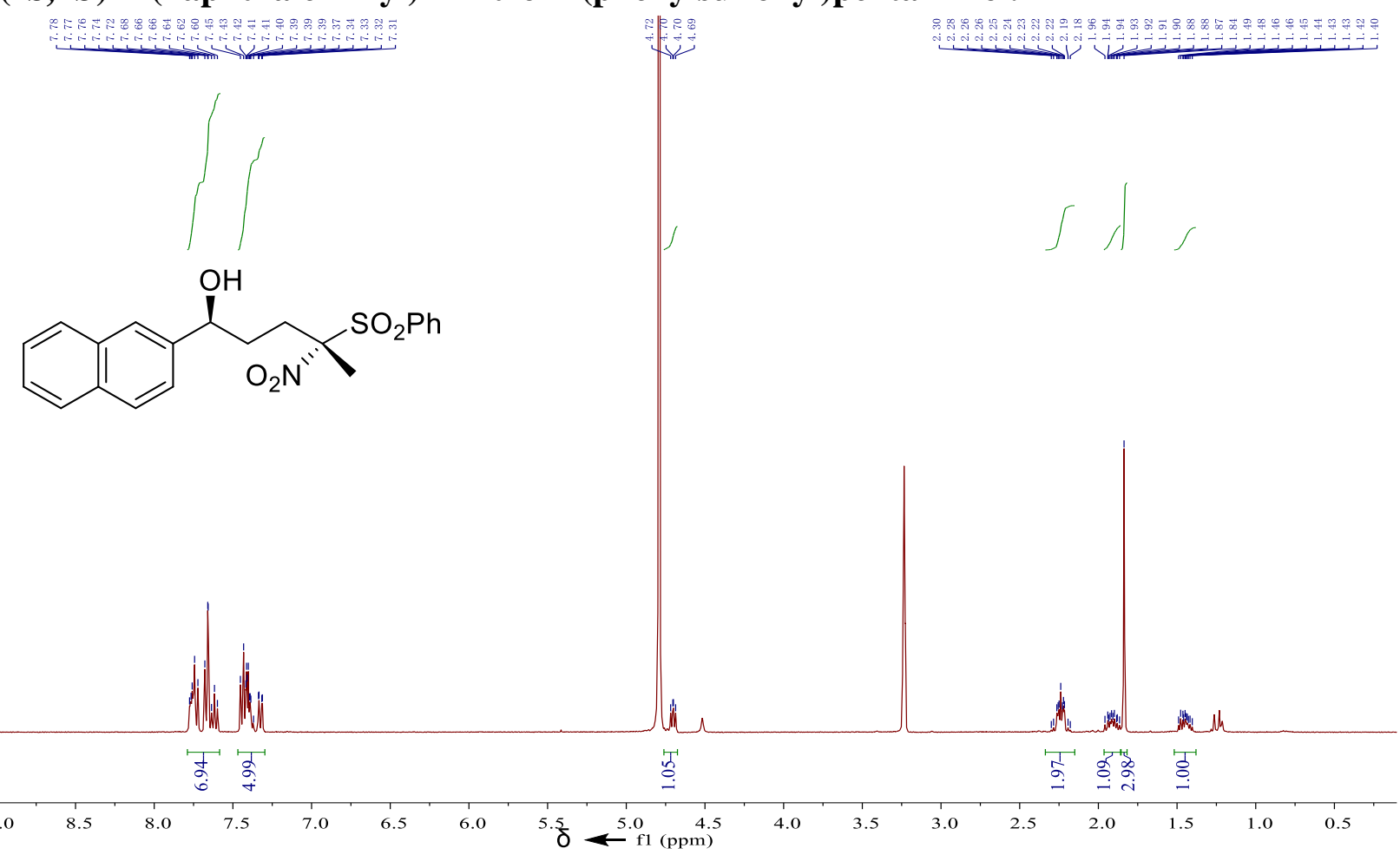

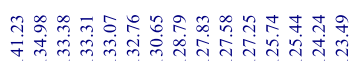

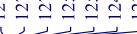<smiles>C[C@](CC[C@H](O)c1ccc2ccccc2c1)([N+](=O)[O-])S(=O)(=O)c1ccccc1</smiles>

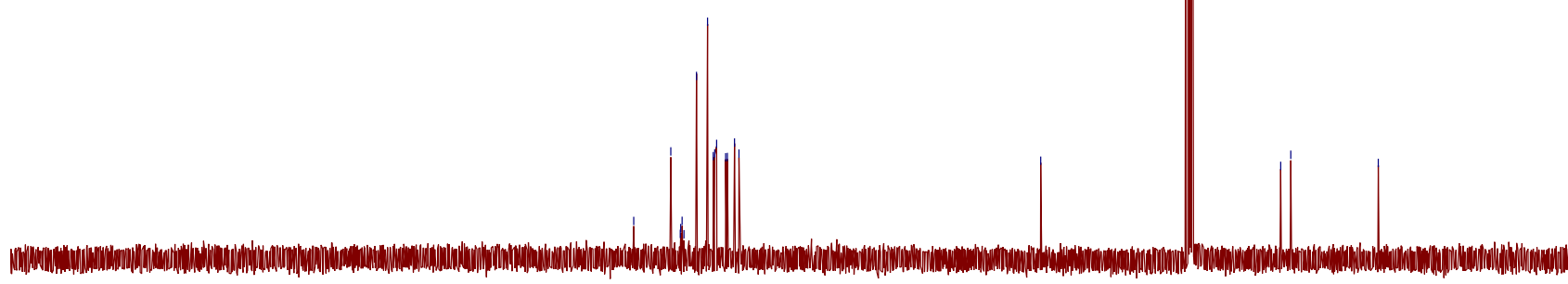

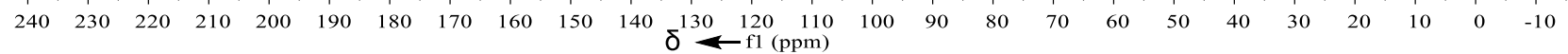


8p: (1S,4S)-1-(furan-2-yl)-4-nitro-4-(phenylsulfonyl)pentan-1-ol.

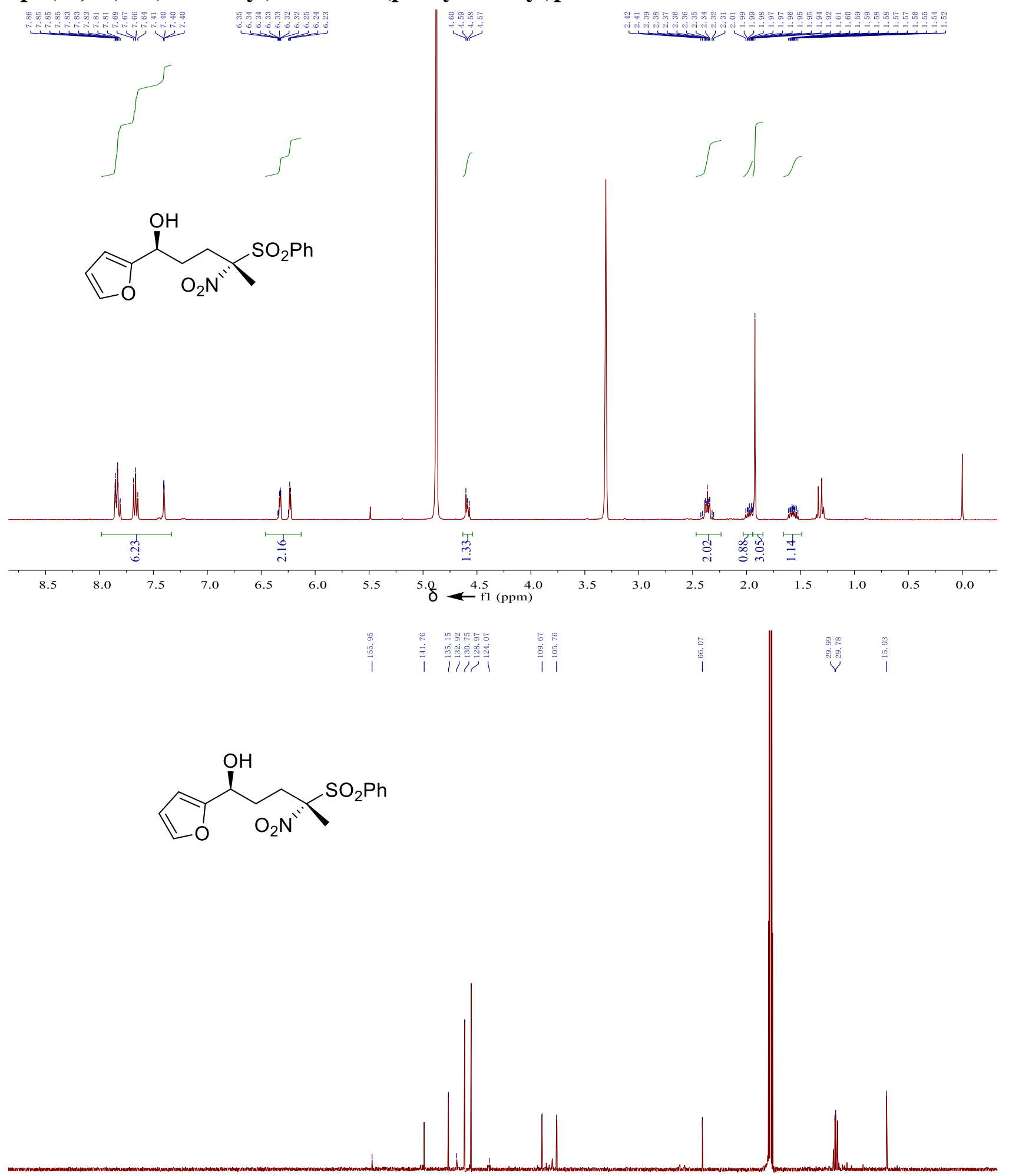

$\begin{array}{llllllllllllllllllllllllllllllllllll}250 & 240 & 230 & 220 & 210 & 200 & 190 & 180 & 170 & 160 & 150 & 140 & 130 & 120 & 110 & 100 & 90 & 80 & 70 & 60 & 50 & 40 & 30 & 20 & 10 & 0 & -10\end{array}$ 
8q: (1S,4S)-4-nitro-4-(phenylsulfonyl)-1-(thiophen-2-yl)pentan-1-ol.

$\underbrace{10}$

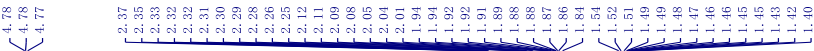<smiles>CC1CCCCCC1</smiles>

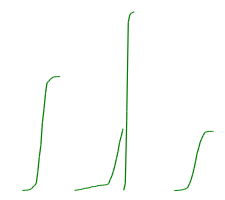<smiles>C[C@](CCC(O)c1cccs1)([N+](=O)[O-])S(=O)(=O)c1ccccc1</smiles>

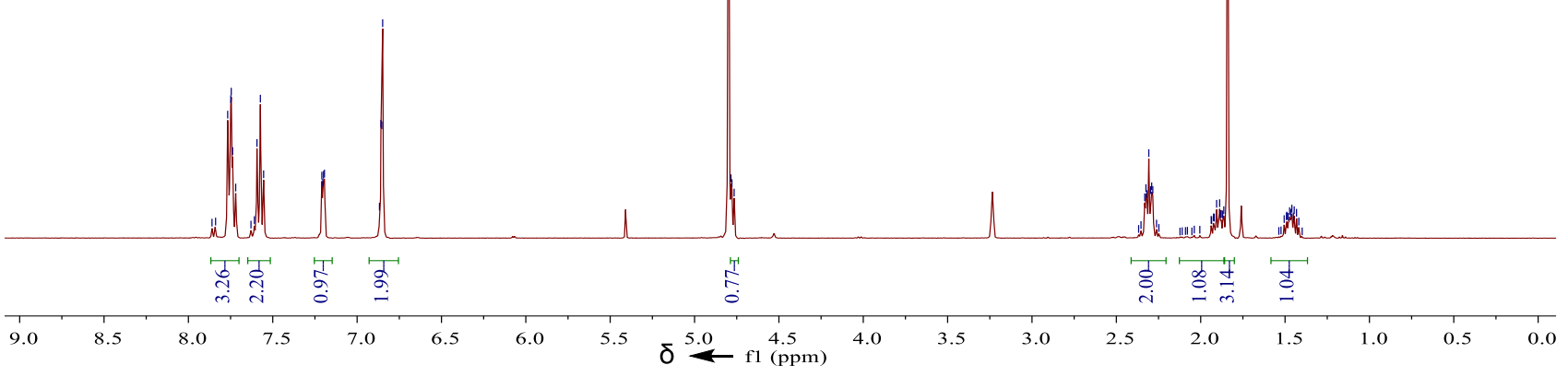<smiles></smiles>

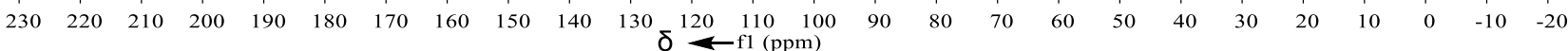


8r: (1S,4S)-1-(3,9-dihydropyren-4-yl)-4-nitro-4-(phenylsulfonyl)pentan-1-ol.

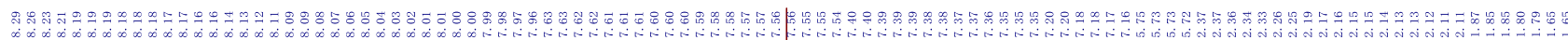

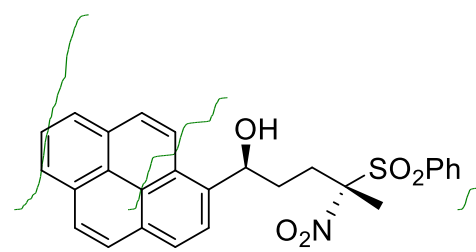
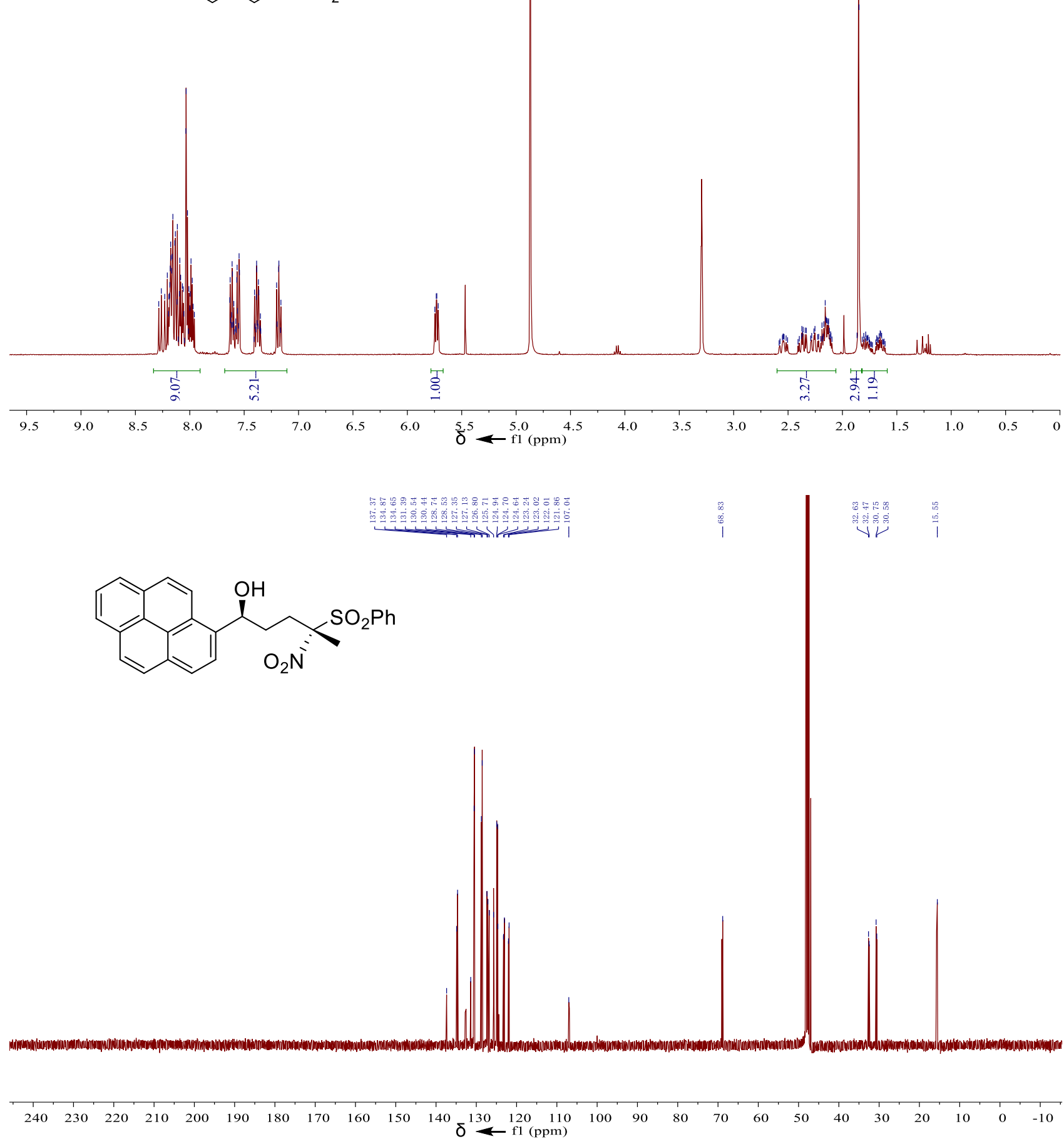

Figure S11. Characterization of chiral products (The ${ }^{1} \mathrm{H}$ NMR and ${ }^{13} \mathrm{C}$ NMR spectra of all chiral products). 